\title{
Femtosecond Laser Micro/Nano-manufacturing: Theories, Measurements, Methods, and Applications
}

\author{
Baoshan Guo ${ }^{1} \mathbb{D} \cdot$ Jingya Sun $^{1} \cdot$ Yanhong Hua ${ }^{1} \cdot$ Ningwei Zhan $^{1} \cdot$ Jingang Jia ${ }^{1} \cdot$ Kunpeng Chu $^{1}$
}

Received: 11 November 2019 / Revised: 15 January 2020 / Accepted: 30 January 2020 / Published online: 28 February 2020

(c) The Author(s) 2020

\begin{abstract}
Femtosecond laser fabrication has grown to be a major method of extreme manufacturing because of the extreme energy density and spatial and temporal scales of femtosecond lasers. The physical effects and the mechanism of interaction between femtosecond lasers and materials are distinct from those in traditional processes. The nonlinear and nonequilibrium effects of the interaction have given rise to new concepts, principles, and methods, such as femtosecond pulse durations are shorter than many physical/chemical characteristic times, which permits manipulating, adjusting, or interfering with electron dynamics. These new concepts and methods have broad application prospects in micro/nanofabrication, chemical synthesis, material processing, quantum control, and other related fields. This review discusses the cutting-edge theories, methods, measurements, and applications of femtosecond lasers to micro/nano-manufacturing. The key to future development of femtosecond laser manufacturing lies in revealing its fabrication mechanism from the electronic level and precisely regulating the electronic dynamics.
\end{abstract}

Keywords Femtosecond laser $\cdot$ Micro/nano-manufacturing $\cdot$ Pulse shaping $\cdot$ Electron dynamics control

\section{Introduction}

In recent years, ultrafast laser micro/nano-manufacturing has become one of the frontiers in the development of manufacturing technology because ultrafast lasers can change the states and properties of materials through the interaction with them and they can be used to control materials for processing from micron to nanometer scales or across scales [1]. Femtosecond lasers tend to impose extreme conditions in the interaction with target materials because of the ultrashort temporal scales $\left(\sim 10^{-15} \mathrm{~s}\right)$ and the high energy density $\left(>10^{14} \mathrm{~W} / \mathrm{cm}^{2}\right)$ while they can be focused into nanometer spatial dimensions $\left(\sim 10^{-9} \mathrm{~m}\right)$. Because of these characteristics, femtosecond lasers can process almost any material with high quality and high precision, and realize threedimensional complex structure fabrication.

The nonlinear (multiphoton, etc.) absorption of a femtosecond laser enables it to break through the limitations

Baoshan Guo

gbs@bit.edu.cn

1 Laser Micro/Nano Fabrication Laboratory, School of Mechanical Engineering, Beijing Institute of Technology, Beijing 100081, China of traditional fabrication methods, and fabrication accuracy currently stands at $1 / 50$ of the diffraction limit [2]. The nonequilibrium (interelectron nonequilibrium, electronto-lattice nonequilibrium, etc.) absorption and nonthermal phase transitions (Coulomb explosion, electrostatic stripping, etc.) of femtosecond lasers can minimize heat-affected zones, cracks, and recast layers, which greatly improves the quality of fabrication [3, 4]. In addition, no mask, vacuum, or reactive gas environment is needed in femtosecond laser fabrication. Furthermore, the process generates particularly low amounts of waste compared with traditional methods and does not cause pollution. These characteristics of femtosecond lasers have led to the development of new manufacturing concepts, principles, methods, and techniques, which support a large number of manufacturing applications, such as process and automation technology, information technology, telecommunications technology, biotechnology, and the pharmaceuticals, aerospace, and environmental industries [5-11]. Meanwhile, the mechanism of femtosecond laser fabrication including phase change and material removal is completely different from the mechanisms of traditional manufacturing methods, which essentially determined by laser-electron interactions. Hence, the regulation of laserelectron interactions or electrons dynamics is critical to the 
future development of femtosecond laser manufacturing, which poses a challenge for measuring and controlling at the electron level during fabrication processes. Hence, the development of theory and observation system must be synchronized with the development of laser fabrication methods and applications.

In this article, we review the progress of femtosecond laser micro/nano fabrication with respect to the aspects of theories, measurements, methods, and applications:

1. Theoretical models of femtosecond laser fabrication, including the first-principles model (the time-dependent density functional theory), molecular dynamics model, improved two-temperature model, plasma quantum model, multi-scale model. The measurements can be realized by a multi-scale observation system integrated with femtosecond laser pump-probe technique, laserinduced breakdown spectroscopy, time-resolved plasma imaging and intensified CCD system.

2. Methods of femtosecond laser fabrication, including femtosecond laser direct writing fabrication, femtosecond laser time-shaping fabrication, femtosecond laser space-shaping fabrication, femtosecond laser frequency-shaping fabrication, femtosecond-laser-induced plasmonic fabrication, and femtosecond-laser-induced chemical fabrication.

3. Applications of femtosecond laser fabrication, including for superhydrophobic surfaces, high-aspect-ratio microholes, laser shock peening, biomedical and protein applications, new functional materials and devices.

\section{Femtosecond Laser Fabrication Theory}

The interaction between a femtosecond laser and materials is an ultrafast nonlinear and nonequilibrium process. In this part, the interaction process between a femtosecond laser and materials is introduced briefly. Then, the main theoretical models of the femtosecond laser-materials interaction are interpreted in detail. On this basis, a multiscale theory and multiscale observation system for femtosecond laser fabrication are introduced.

\subsection{Interaction of Femtosecond Lasers with Metals and Nonmetals}

Metallic materials have a large number of free electrons. When a femtosecond laser interacts with metallic materials, free electrons absorb photon energy and are heated. They collide with other electrons to transfer energy to each other first. As the number of heated electrons increases, the interaction between electrons and the lattice intensifies, and finally, the lattice is heated. After the lattice is heated, the material will undergo phase transition at the picosecond $\left(10^{-12} \mathrm{~s}\right)$ to tens of nanoseconds $\left(10^{-8} \mathrm{~s}\right)$ scale, resulting in microsecond- to millisecond-scale plasma. Unlike metallic materials, nonmetallic materials do not have adequate free electrons. Most electrons are bound in the valance band, because of which the femtosecond laser ablation mechanism of nonmetallic materials is different from that of metallic materials. Femtosecond laser processing of nonmetallic materials is mainly divided into two parts. The first part is the ionization, which involves the photoionization and impact ionization mechanisms. The photoionization mechanism comprises tunnel ionization and multiphoton ionization. Multiphoton ionization refers to the valence-band electrons absorb multiple photon energies and transfer into the conduction band, becoming free electrons in the process [12]. A schematic of this process is shown in Fig. 1a. Tunnel ionization is caused by the super-strong electromagnetic field generated when a femtosecond laser acts on a nonmetallic material, which reduces the Coulomb potential barrier of valence-band electrons. As shown in Fig. 1b, valenceband electrons can pass through the Coulomb barrier by means of the tunneling effect, transfer into the conduction band, and become free electrons.

Impact ionization is different from photoionization. When low-energy free electrons absorb more than one photon and transform into high-energy free electrons in the conduction band, the free electrons obtain higher levels of kinetic energy and collide with other valence electrons. In this case, the energy is transferred to generate two low-energy free electrons. These low-energy free electrons also absorb photons and then collide and ionize again, causing a chain reaction very similar to an avalanche. Therefore, impact ionization (a)

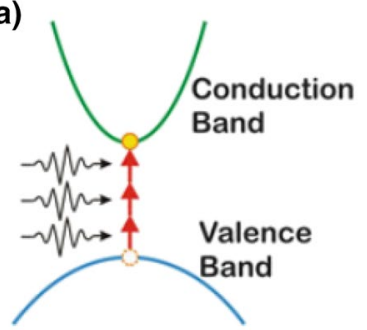

(c)
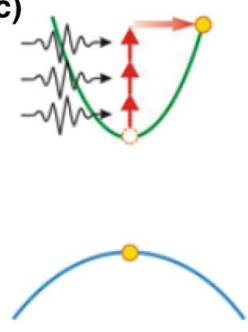

(b)
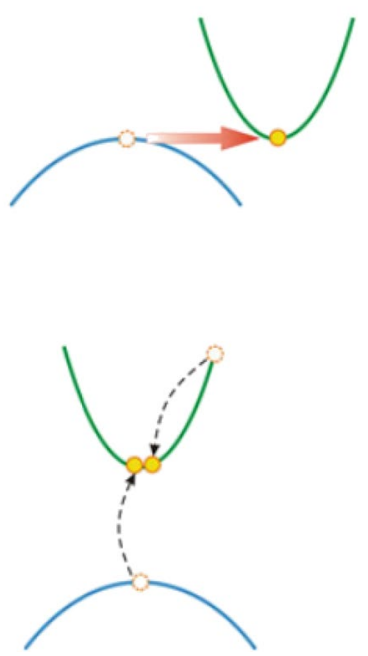

Fig. 1 Schematic of a multiphoton ionization, b tunnel ionization, and $\mathbf{c}$ avalanche ionization [13] 
is also called avalanche ionization [12]. A schematic of the entire process is shown in Fig. 1c. Photoionization provides seed electrons for impact ionization. Early studies on the topic found that the impurities or defect states in materials could be thermally stimulated to provide seed electrons for impact ionization, but this process generates very few seed electrons [13]. Photoionization is the main mechanism of the seed-electron generation.

The second part is the phase change. After the first part of ionization and electron heating, a large number of free electrons accumulate inside the material, and as a result, a nonmetallic material exhibits transient metallic characteristics. The interaction of free electrons with the lattice may causes a phase change. The phase-transition mechanisms include thermal phase transitions (melting and gasification) and nonthermal phase transitions (Coulomb explosions and electrostatic ablation). When the lattice temperature rises above the melting point of the material, melting becomes the main phase transition mechanism of the material. The phase change of a nonthermal phase transition is not based on lattice heating but on plasma expansion. When a nonmetallic material ionizes free electrons instantaneously under the radiation of a femtosecond laser, plasma density inside the material increases rapidly and the plasma oscillation frequency gradually approaches the laser frequency. According to Stoian et al. [14], the nonthermal phase transition mechanism of Coulomb explosion dominates the phase transition process of materials.

\subsection{Theoretical Models of Femtosecond Laser Fabrication}

As mentioned earlier, the interaction of a femtosecond laser with materials is a complex and ultrafast nonlinear process. Different theoretical models have their own limitations in the temporal and spatial scales. It is difficult to fully explain the entire process on the basis of a single theoretical model. Therefore, the entire laser and material interaction system is divided into several subsystems, including electronic systems, ionic or molecular systems, and plasma systems, to simplify the process of theoretical interpretation. In this section, the first principles, molecular dynamics, plasma quantum, and improved two-temperature models are introduced for use in theoretically interpreting different subsystems. Thereafter, a relatively complete theoretical system detailing the interaction between a femtosecond laser and materials is established.

\subsubsection{First Principles (Time-Dependent Density Functional Theory)}

During the initial interaction of a femtosecond laser with a material, laser energy is absorbed by the material through photon and electron interactions. To understand this nonlinear process, first-principles calculation methods are used to simplify the entire physical system into a series of nuclear and electronic systems that rely on the interaction of electric fields. The problem is further simplified by adiabatic and classical approximations of the multibody Schrödinger equation. Finally, by using the time-dependent density functional theory (TDDFT), a series of noninteracting timedependent Kohn-Sham single-particle equations with electron density as an independent variable are solved. Thereby, the electronic state of a transient state during the action of the femtosecond laser is obtained. The time-dependent Kohn-Sham single-particle equation is given below:

$i \hbar \frac{\partial}{\partial t} \psi_{i}(\vec{r}, t)=H_{\mathrm{KS}}(\vec{r}, t) \psi_{i}(\vec{r}, t), n(\vec{r}, t)=\sum_{i}\left|\psi_{i}(\vec{r}, t)\right|^{2}$

where $n(\vec{r}, t)$ is the electron density. $H_{\mathrm{KS}}(\vec{r}, t)$ is the Kohn-Sham Hamiltonian and is expressed as follows:

$$
\begin{aligned}
H_{\mathrm{KS}}(\vec{r}, t) & =\frac{1}{2 m}\left(\vec{p}+\frac{e}{c} \vec{A}_{\mathrm{tot}}(t)\right)^{2} \\
& +V_{\text {ion }}(\vec{r}, t)+e^{2} \int \mathrm{d} \frac{\vec{r}}{\left|\vec{r}-\vec{r}^{\prime}\right|}+V_{x c}(\vec{r}, t)
\end{aligned}
$$

where $e$ is the amount of elemental charge, $V_{\text {ion }}(\vec{r}, t)$ is the electron-ion potential, $V_{x c}(\vec{r}, t)$ is the exchange correlation potential, and $\vec{A}_{\text {tot }}(t)$ is the time-dependent spatially uniform vector potential. $A_{\text {tot }}(t)$ consists of an external and induced vector potential; that is, $\vec{A}_{\text {tot }}(t)=\vec{A}_{\text {ext }}(t)+\vec{A}_{\text {ind }}(t)$. The external vector potential is formed by the electromagnetic field generated by the laser.

$\vec{E}_{\text {laser }}(t)=-\mathrm{d} \vec{A}_{\text {ext }}(t) / \mathrm{d} t$

$E_{\text {laser }}(t)=E_{0} \sin ^{2}\left(\frac{\pi t}{T}\right) \sin (\omega t), \quad(0<t<T)$.

The induced vector potential can be obtained from Eq. (2-5):

$\frac{\mathrm{d}^{2} \vec{A}_{i n d}(t)}{\mathrm{d} t^{2}}=\frac{4 \pi}{c} \vec{i}(t)$

where $\vec{i}(t)$ is the average current density.

The evolution of the wave function over a short time, $\Delta t$, can be calculated by Eq. (2-6):

$\psi(\vec{r}, t+\Delta t)=e^{-i \hat{H}_{\mathrm{KS}}(t+\Delta t) \frac{\Delta t}{2}} e^{-i \hat{H}_{\mathrm{KS}}(t) \frac{\Delta t}{2}} \psi(\vec{r}, t)$ 
By solving the multi-body time-dependent Schrödinger equation, the evolution of the electron density of the femtosecond laser in the material can be obtained, regulation of the femtosecond laser to the transient electronic state can be achieved, and optical properties of the material can be determined. This proves that it is feasible to electronically control femtosecond laser fabrication.

On the basis of the TDDFT, the electronic dynamics of silica irradiated by a time-shaped femtosecond laser have been calculated and simulated [15]. It has been proven theoretically that an increase in the delay time of a femtosecond laser pulse in the range of 10-30 fs will increase the number of exciting electrons and greatly improve the absorption efficiency of laser energy. Time-varying density functional calculations of the ultrafast kinetics of a phosphite monolayer irradiated by femtosecond laser have been carried out [16]. It was found that under femtosecond laser irradiation, the real part of the phosphonene dielectric function diverges negatively at low laser frequencies and the imaginary part exhibits a significant "quasi-exciton" absorption peak (Fig. 2). It was inferred that electron-hole pairs excited by a femtosecond laser are responsible for this experimental phenomenon.

First-principles calculation based on the TDDFT can explain the photon-electron interaction process between a femtosecond laser and a material, reveal the ionization mechanism of the material, and help obtain the electronic state and optical properties of the material transient, which are the foundation for the theoretical calculation of the subsequent process.

\subsubsection{Molecular Dynamics Model}

By solving Newton's mechanical equations about a series of molecular motions, motion parameters such as displacement and velocity of the molecular system are obtained, and thermodynamic parameters of the system are further calculated. During the interaction between a femtosecond laser and a material, molecular dynamics models often combine thermodynamic models, such as the two-temperature model, to solve the temperature change process of the material lattice. The calculation process is as follows:

$m_{i} \frac{\mathrm{d} \vec{r}_{i}}{\mathrm{~d} t^{2}}=\vec{f}_{i}, \vec{f}_{i}=-\frac{\partial \vec{U}}{\partial t}$

where $\vec{r}_{i}$ is the position coordinate of the $i$ th atom and $\vec{f}_{i}$ is the force acting on it. It is solved by the potential function acting on the entire system. The potential function selected

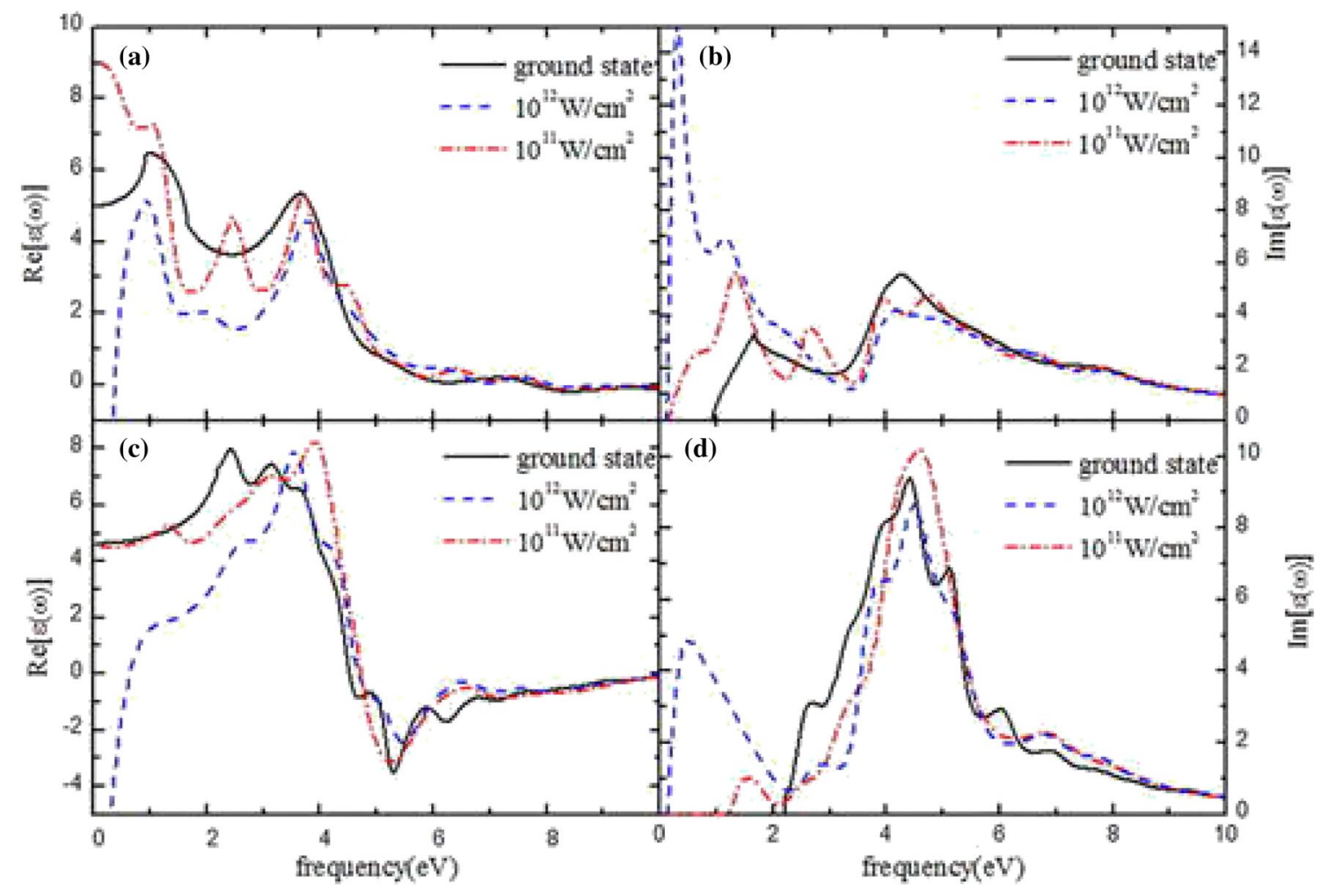

Fig. 2 Real (a, c) and imaginary (b, d) parts of the dielectric function of monolayer phosphorene excited by femtosecond laser with intensities of $10^{12} \mathrm{~W} / \mathrm{cm}^{2}$ and $10^{11} \mathrm{~W} / \mathrm{cm}^{2}$. The real part of the dielectric function diverges negatively at low frequencies and a quasi-exciton absorption peak appears in the imaginary part [16] 
in the molecular dynamics calculation simulation process greatly influences simulation accuracy. In the interaction between a femtosecond laser and a material, the Morse potential function is adopted for considering the short-range van der Waals force [17]. The nonbonding potential function $U(r)$ that represented by the Coulomb potential function under long-range Coulomb force is given by Eq. (2-8):

$U(r)=\frac{q_{1} q_{2}}{4 \pi \varepsilon r}+D_{0}\left[e^{\gamma\left(1-r / r_{0}\right)}-2 e^{\gamma / 2\left(1-r / r_{0}\right)}\right]$

In the Morse potential function part of this equation, $D_{0}$ is the total dissociation energy, $\gamma$ is a constant, and $r_{0}$ denotes equilibrium position coordinates.

By using the molecular dynamics model, molecular dynamics calculations and simulations were performed to prepare metal nanoparticles from nickel films irradiated with femtosecond laser pulses [18]. The results showed that the pulse delay time and the number of subpulses controlled the size of the nanoparticles, which further verified the correctness of the theoretical model.

The molecular dynamics model can be used to calculate and explain the lattice phase transition mechanism of the interaction of a femtosecond laser with a material, which is very important for further research on femtosecond laser fabrication (Fig. 3).

\subsubsection{Plasma Model}

The plasma model indicates the process of plasma generation and evolution. After understanding the ionization mechanism in the interaction between a femtosecond laser and a material, the ionization rate equation is obtained through quantum processing combined with the Boltzmann equation. Variation of the free electron density during phase transition of the material is calculated, and optical characteristics of the plasma complex dielectric function are derived. The calculation process is as follows:

Under femtosecond laser irradiation, the distribution of free electrons in a dielectric material can be solved using the Fokker-Planck equation:

$\frac{\partial n_{\mathrm{e}}(t, r, z)}{\partial t}=\alpha_{i} I(t, r, z) n_{e}(t, r, z)+\delta_{N}(I(t, r, z))^{N}-\frac{n_{\mathrm{e}}(t, r, z)}{\tau}$

where $t$ is time, $r$ the distance to the central axis of the Gaussian beam, $z$ the depth from the surface of the material, $n_{e}(t, r, z)$ the free electron density, $I(t, r, z)$ the laser light field intensity, $\tau$ the time in which free electrons recombine with the ground state, $\alpha_{i}$ the impact ionization constant, and $\delta_{N}$ the N-photon absorption cross section.

The optical properties of the material may be characterized by complex dielectric functions by means of the Drude model. The plasma complex dielectric function is expressed as follows:

$\varepsilon(t, r, z)=1-\frac{\omega_{\mathrm{p}}^{2}\left(n_{\mathrm{e}}\right) \tau_{\mathrm{e}}^{2}}{1+\omega^{2} \tau_{\mathrm{e}}^{2}}+i\left(\frac{\omega_{\mathrm{p}}^{2}\left(n_{\mathrm{e}}\right) \tau_{\mathrm{e}}^{2}}{\omega\left(1+\omega^{2} \tau_{\mathrm{e}}^{2}\right)}\right)$

where $\omega_{\mathrm{p}}$ is the plasma oscillation frequency and $\tau_{\mathrm{e}}$ the electron relaxation time, which is using the Boltzmann transport equation:

$\omega_{\mathrm{p}}\left(n_{\mathrm{e}}\right)=\sqrt{n_{\mathrm{e}}(t, r, z) e^{2} / m_{\mathrm{e}} \varepsilon_{0}}$

$\tau_{\mathrm{e}}(t, r, z)=\frac{3 \sqrt{m_{\mathrm{e}}}\left(k_{\mathrm{B}} T_{\mathrm{e}}(t, r, z)\right)^{3 / 2}}{2 \sqrt{2} \pi\left(Z^{*}\right)^{2} n_{\mathrm{e}}(t, r, z) e^{4} \ln \Lambda}$
$\left(1+e^{\left(-\mu\left(n_{\mathrm{e}}, T_{\mathrm{e}}\right) / k_{\mathrm{B}} T_{\mathrm{e}}(t, r, z)\right)}\right) F_{1 / 2}$

where $Z^{*}$ is the number of ionization states, $m_{\mathrm{e}}$ the electron mass, $k_{\mathrm{B}}$ the Boltzmann constant, $F_{1 / 2}$ the Fermi-Dirac integral, $\mu$ the chemical potential energy, and $\ln \Lambda$ the Coulomb logarithm.

Plasma models can be used to describe photon absorption, plasma generation, and recombination of electronic systems before lattice phase transitions. By using the plasma model, Yuan et al. [19-21] predicted the entire process of adjusting femtosecond laser ablation morphology and subwavelength ripple structures by adjusting plasma distribution inside materials through computational simulation (Fig. 4). They further proved the feasibility of controlling femtosecond laser fabrication by means of electronic dynamic control.

\subsubsection{Improved Two-Temperature Model}

The two-temperature model (TTM) was originally proposed by Anisimov et al. [22]. They calculated variation of the electron temperature and lattice temperature in the entire process with respect to photon-electron, electron-electron, electron-lattice, and lattice-lattice interactions. The equations expressing electron and lattice temperature variations are as follows:

$C_{\mathrm{e}}\left(T_{\mathrm{e}}\right) \frac{\partial T_{e}}{\partial t}=K_{\mathrm{e}} \nabla^{2} T_{\mathrm{e}}-G\left(T_{\mathrm{e}}-T_{1}\right)+Q(r, t)$

$C_{1} \frac{\partial T_{1}}{\partial t}=K_{1} \nabla^{2} T_{1}+G\left(T_{\mathrm{e}}-T_{1}\right)$

where $C_{\mathrm{e}}$ and $C_{1}$ represent the electron heat capacity and lattice heat capacity, respectively; $T_{\mathrm{e}}$ and $T_{1}$ represent the electron and lattice temperatures, respectively; $G$ represents the coupling coefficient of the electron and the lattice; $K_{\mathrm{e}}$ represents electron thermal conductivity; and $Q$ represents the unit volume laser input energy. $Q(r, t)$ represents the 

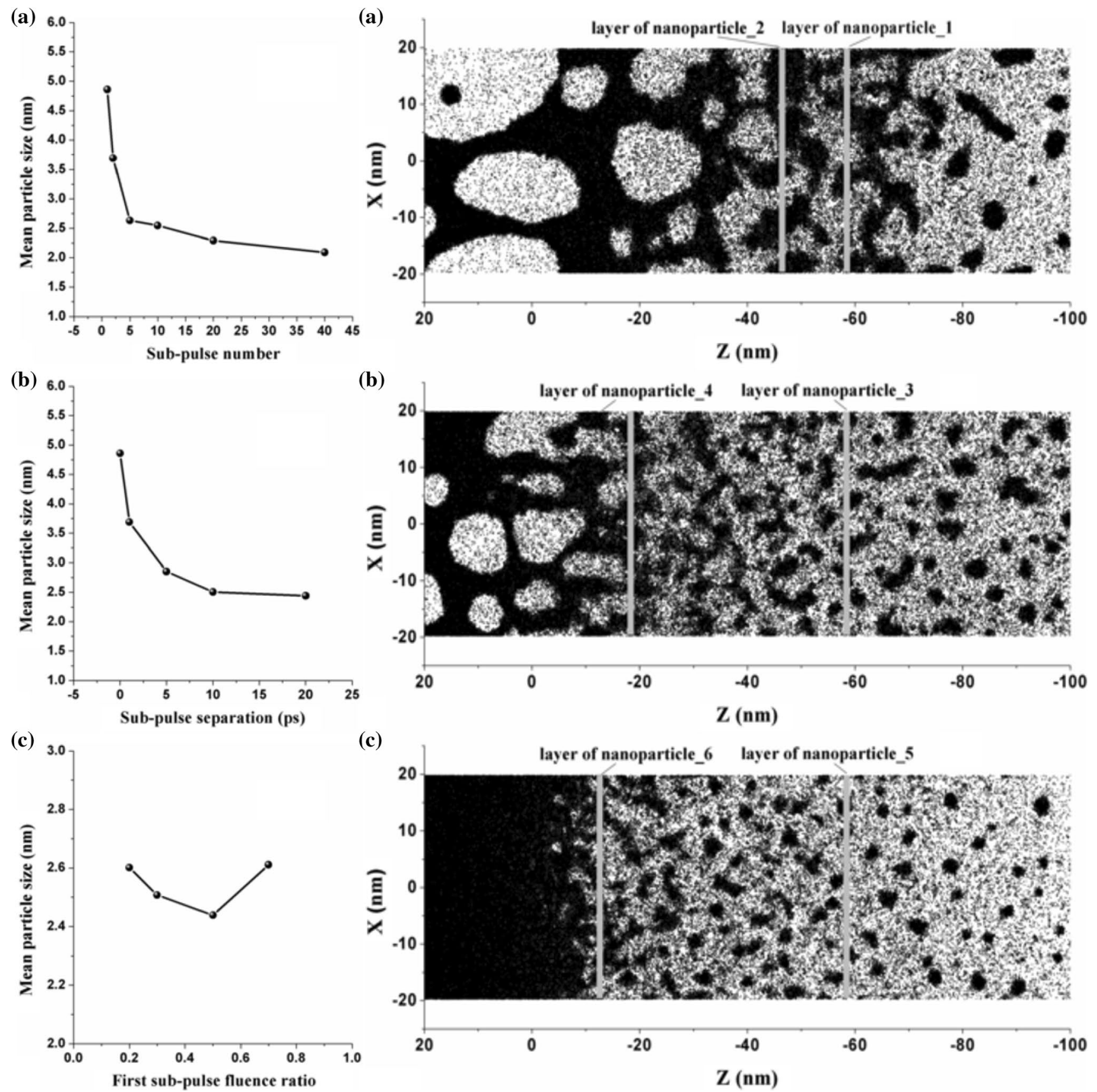

Fig. 3 Simulation results of mean particle size of Ni nanoparticles with a different subpulse numbers, b different subpulse separation times, and c different subpulse fluence ratios. Snapshots of nanoparticles generated under different subpulse numbers [18]

photon and electron interaction process, $K_{\mathrm{e}} \nabla^{2} T_{\mathrm{e}}$ the electron and electron interaction process, $G\left(T_{\mathrm{e}}-T_{1}\right)$ the electron and lattice interaction process, and $K_{1} \nabla^{2} T_{1}$ the lattice and lattice interaction process.

The classical TTM is unsuitable for representing the interaction between a femtosecond laser and a metal under high laser flux. Jiang et al. improved the classic TTM and expanded its range of applications [23]. The improvements included (1) calculation of the heat capacity of free electrons from Fermi distribution, (2) determination of free electron relaxation time and electron thermal conductivity by solving the plasma Boltzmann transport equation, and (3) calculation of optical properties such as reflectance and absorption coefficient by using the improved Drude model that considers free electron heating and interband transition. By using the improved TTM, the electron and phonon temperatures of gold films irradiated by femtosecond laser 

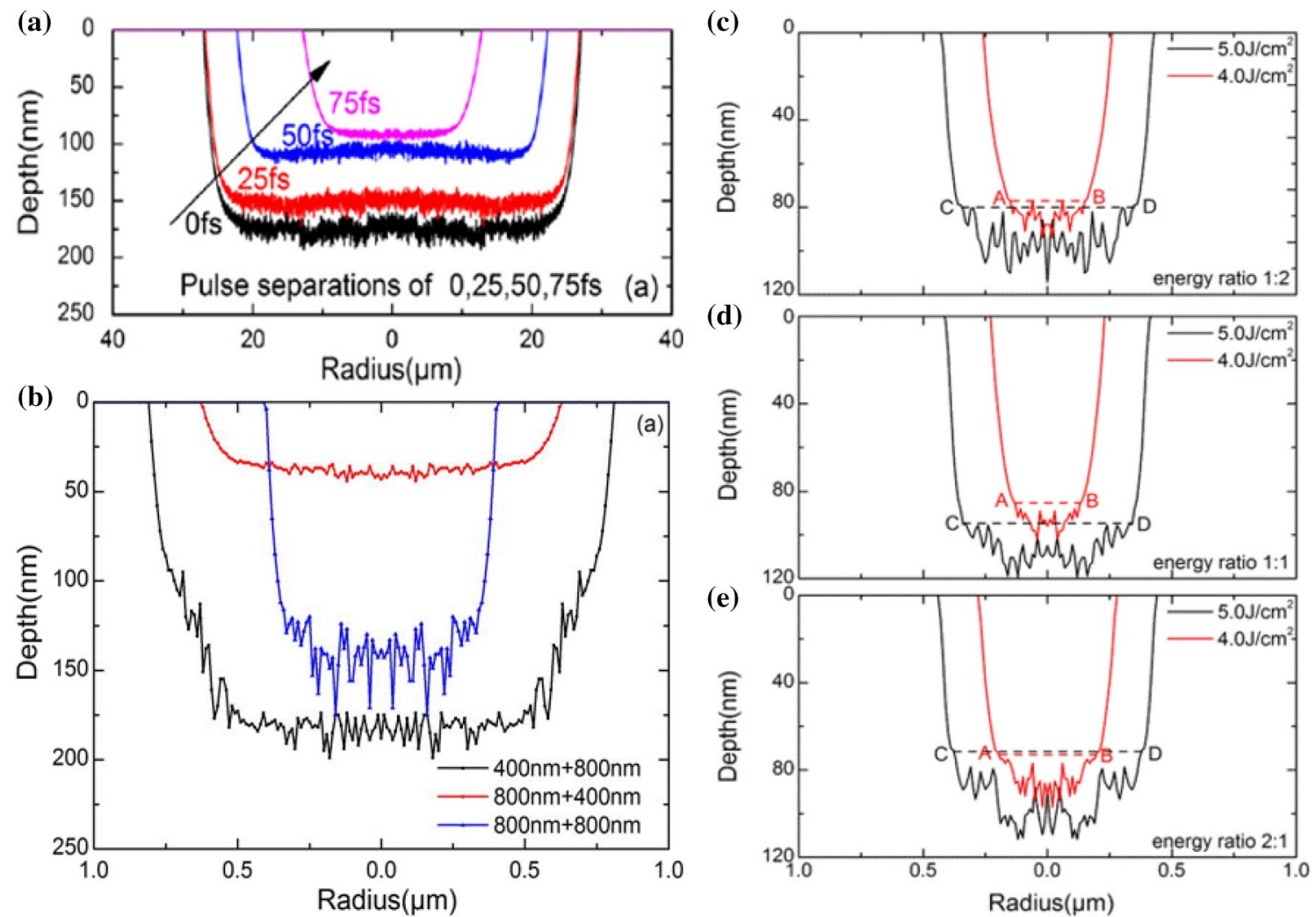

Fig. 4 Shape of ablation crater under different double femtosecond laser pulse radiations predicted with plasma quantum models: a different delay times, $\mathbf{b}$ different wavelengths, and $\mathbf{c}-\mathbf{e}$ different laser intensities and energy ratios $[19,21]$

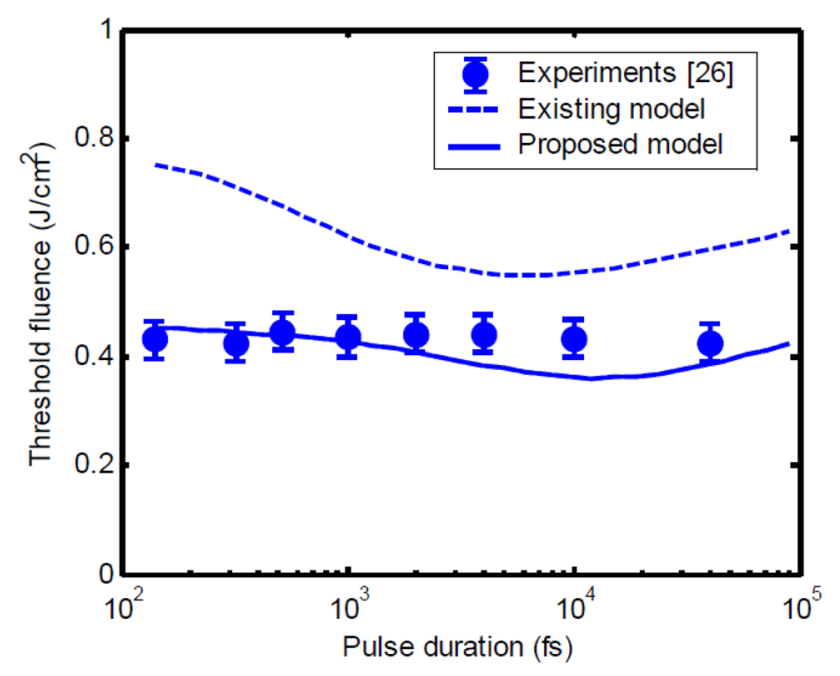

Fig. 5 Curves of pulse duration and threshold fluence of experimental and theoretical results [23]

can be calculated theoretically and verified experimentally $[23,24]$. The experimental results show that the improved TTM yields a more accurate theoretical prediction of the ablation threshold of the femtosecond laser-gold film interaction compared with the conventional model (Fig. 5).

Lutz et al. [25] improved the TTM with ab initio method to calculate the parameters in the model except the electron-phonon coupling strength, which is obtained from density functional theory molecular dynamics simulations. A nonthermal lattice model (NLM) was also introduced for describing nonthermal phonon distributions (Fig. 6). The development of TTM is summarized in Table 1.

\subsection{Multiscale Model and Observation Systems}

Each model has its own limitations with respect to explaining the mechanisms of interaction of a femtosecond laser with a material. In the two-temperature model, electron density is set to a constant value; thus, the two-temperature model can only be applied to study the mechanism of interaction between a femtosecond laser and metallic materials. The plasma model offers a good explanation for the nonmetallic ionization process. However, the plasma model simply describes the phase ablation process of the material as the plasma density reaches the critical density; that is, when the plasma oscillation frequency is consistent with the laser frequency, ablation will occur. The phase transition process 
(a)

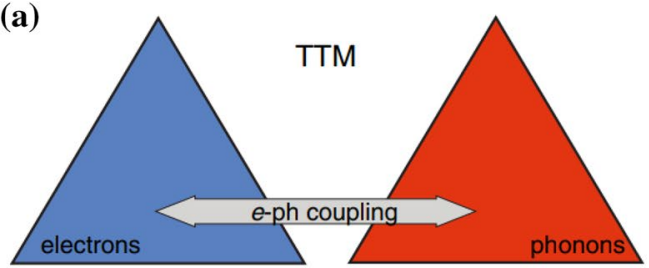

(b)

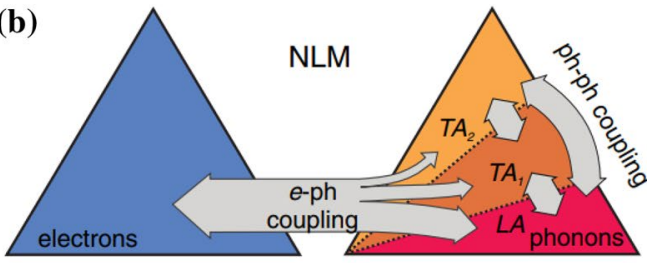

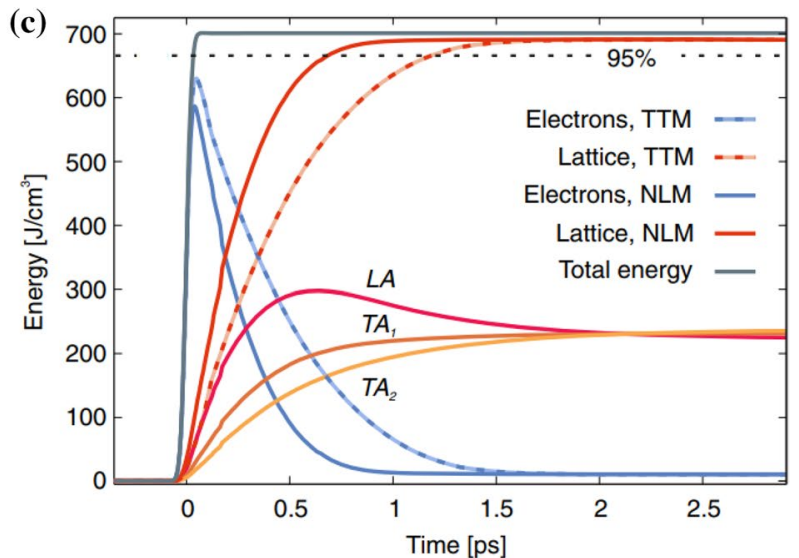

Fig. 6 Schematic representation of the TTM and the NLM and temporal evolution of the energy content in the electronic (dash-dotted lines) and lattice (solid lines) subsystems for an excitation density of $680 \mathrm{~J} / \mathrm{cm}^{3}$ according to the NLM and the TTM [25]

Table 1 Development of TTM

\begin{tabular}{|c|c|c|}
\hline Year & Improvements or results & References \\
\hline 1974 & Separating the heat capacity of metals into lattice and electronic parts & [22] \\
\hline 1996 & $\begin{array}{l}\text { Electrons under non-equilibrium condition (i.e., non-Fermi distribution) relax via electron-phonon coupling in } 2.5 \mathrm{ps,} \text { and } \\
\text { the phonon relaxation of the lattice occurs in }>50 \mathrm{p}\end{array}$ & [26] \\
\hline 1999 & Emphasizing the importance of electron-phonon coupling in the phase changing process & [27] \\
\hline 2002 & $\begin{array}{l}\text { Using Boltzmann collision integral but not relaxation time approach to describe the absorption process. Definite the ther- } \\
\text { malization time as the time after the thermalized limit }\end{array}$ & [28] \\
\hline 2005 & $\begin{array}{l}\text { Extending the existing two-temperature model to high electron temperatures by using full-run quantum treatments to calcu- } \\
\text { late the significantly varying properties }\end{array}$ & [23] \\
\hline 2007 & Introducing the TTM into electron-phonon-magnon systems & [29] \\
\hline 2007 & Coupling TTM with an interfacial tracking method to investigate the ultra-fast phase-change process & [30] \\
\hline 2010 & $\begin{array}{l}\text { Coupling TTM with the Landau-Lifshitz-Bloch equation for a macrospin (containing the statistically averaged magnetic } \\
\text { fluctuations) to explain thermal mechanisms in laser-induced femtosecond spin dynamics }\end{array}$ & [31] \\
\hline 2011 & $\begin{array}{l}\text { Using TTM to interpret the dynamics of the localized plasmon resonance exhibited by colloidal } \mathrm{Cu}_{2-x} \text { Se nanocrystals } \\
\text { excited by ultrafast laser pulses }\end{array}$ & [32] \\
\hline 2011 & $\begin{array}{l}\text { Describing the TTM with entropy and phonon coordinate and form a thermo-dynamical model to understand the coherent } \\
\text { excitation of optical phonons }\end{array}$ & [33] \\
\hline 2013 & $\begin{array}{l}\text { Using TTM to calculate the nonlinear generation of conduction band electrons in silicon and their relaxation to investigate } \\
\text { the formation mechanism of near-wavelength laser-induced periodic surface structures (LIPSS) on silicon }\end{array}$ & [34] \\
\hline 2016 & $\begin{array}{l}\text { Implying ab initio theory to calculate the parameters in TTM and introduce a nonthermal lattice model (NLM) for describing } \\
\text { nonthermal phonon distributions }\end{array}$ & [25] \\
\hline
\end{tabular}

is not discussed in detail. The molecular dynamics model explains the process of material ablation due to lattice phase transition with the thermal and nonthermal phase transition mechanisms. However, the interaction force between atoms changes sharply during the ablation process of a femtosecond laser. The potential function adopted in the molecular dynamics model is relatively simple. Moreover, in the firstprinciples model, many approximate simplifications of the model are carried out, such as a time-dependent exchange correlation, and both of them influence the accuracy of theoretical predictions.
Owing to the limitations of various theoretical models, the multiscale theoretical model and observation system were proposed to describe and measure the interaction between a femtosecond laser and a material, as shown in Fig. 7 [35].

In the temporal scale of picoseconds-to-tens-of-nanoseconds and the spatial scale of tens-of-nanometers-to-tens-ofmicrometers, nonthermal phase transitions such as Coulomb explosion and electrostatic ablation and thermal phase transformation processes such as melting and gasification occur inside the material. Simultaneously, early plasma generation and shock wave propagation begins. The femtosecond laser 


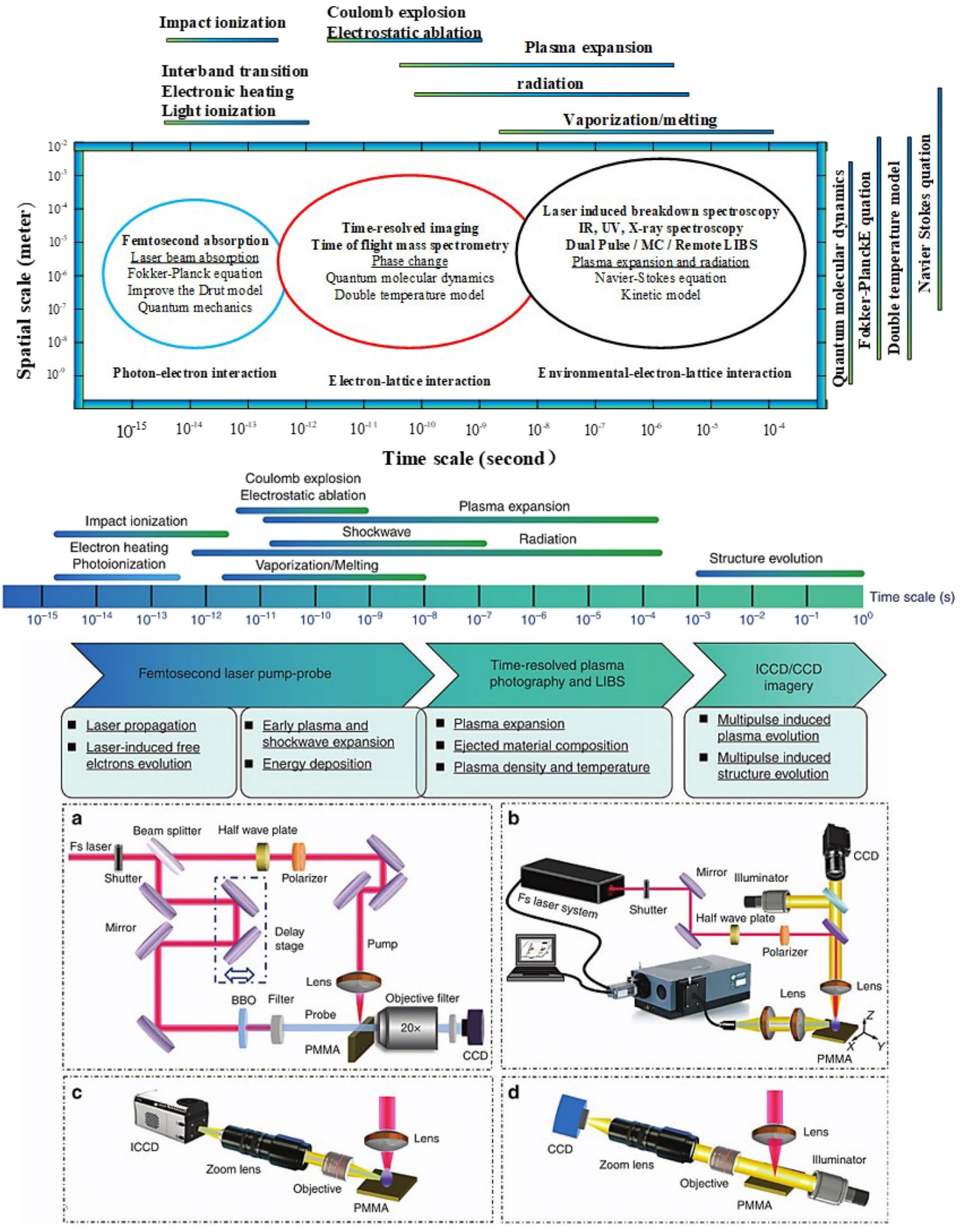

Fig. 7 Schematic of multiscale model and observation system [35] 
pump-probe technique can be used to observe the ablation processes in this temporal and spatial scale. Plasma processes can be observed using time-resolved plasma imaging and laser-induced breakdown spectroscopy (LIBS). In the nanosecond and longer time scales, evolution of the micro/ nano structure occurs on the material surface. In this process, the influence of the laser on plasma distribution leads to the formation and evolution of the surface micro/nanostructure, which can be explained using the theory of surface plasmon scattering and can be observed with an intensified CCD (ICCD). In this process, the laser field and the surface plasmons interact on the material surface.

An imaging system based on temporal wavelength division is proposed to be integrated with the femtosecond laser fabrication system to realize real-time continuous observation and feedback of the fabrication process (Fig. 8) [36]. A representative technique of the continuous imaging is the sequentially time all-optical mapping photography (STAMP) [37].

The multiscale observation system can be used to study the problems associated with each temporal scale to cover the overall scope of laser fabrication. For example, on the basis of the two-color ( $400 \mathrm{~nm}$ and $800 \mathrm{~nm}$ ) pump-probe technique, Pan et al. [38] investigated the temporal and spatial evolution of the plasma induced by a femtosecond laser in fused silica (Fig. 9). Experimental results of the electron relaxation time and electron density fit well with the theoretical predictions, thus validating the plasma model. Kübel et al. [39] investigated the valence electron motion in argon ion with spatiotemporal imaging based on ultrafast pumpprobe technology (Fig. 10).

To improve the ultrafast pump-probe technology, the multiple synchronized, ultrashort, high-energy beams are utilized. Wenz et al. [40] developed a compact laser-driven accelerator to simultaneous generate twin-electron beams as showed in Fig. 11.
In addition, to investigate the LIBS enhancement mechanism of double femtosecond pulses irradiated on polymethyl methacrylate (PMMA), Wang et al. [41] conducted related experiments to investigate the characteristics of laser-excited plasma under single and double pulses (Fig. 12). By measuring each peak value in the LIBS, the electron density of each type of plasma can be calculated at different delay times. In this way, the evolution of electron density in the plasma generated by femtosecond laser irradiation can be investigated to reveal the generation and evolution mechanisms of the plasma in femtosecond laser fabrication.

Wang et al. [42] explored the effects of initial sample temperatures on the femtosecond laser inducing Ge plasma. The experiment results showed that the spectral intensity of the Ge plasma decreased with the initial temperature increasing. To understand the experiment result, ultrafast time-resolved pump-probe experiment was presented to investigate the changing process of the transient Ge plasma relative reflectivity (Fig. 13).

Effects of microstructure and surface impurity segregation which detected by femtosecond LIBS of Al-substituted $\mathrm{Li}_{7} \mathrm{La}_{3} \mathrm{Zr}_{2} \mathrm{O}_{12}$ (LLZO) on total ionic conductivity and interfacial resistance were explored by Cheng et al. [43].

\section{Femtosecond Laser Fabrication Methods}

In addition to the development of theories and observation systems, femtosecond lasers have achieved wide applications in micro/nano fabrication and manufacturing. Presently, the main femtosecond laser micro/nano manufacturing methods include femtosecond laser direct writing, femtosecond laser pulse shaping in the temporal domain, femtosecond laser pulse shaping in the spatial domain, femtosecond laser frequency modulation, and

Fig. 8 Schematic of femtosecond laser fabrication and realtime continuous imaging system

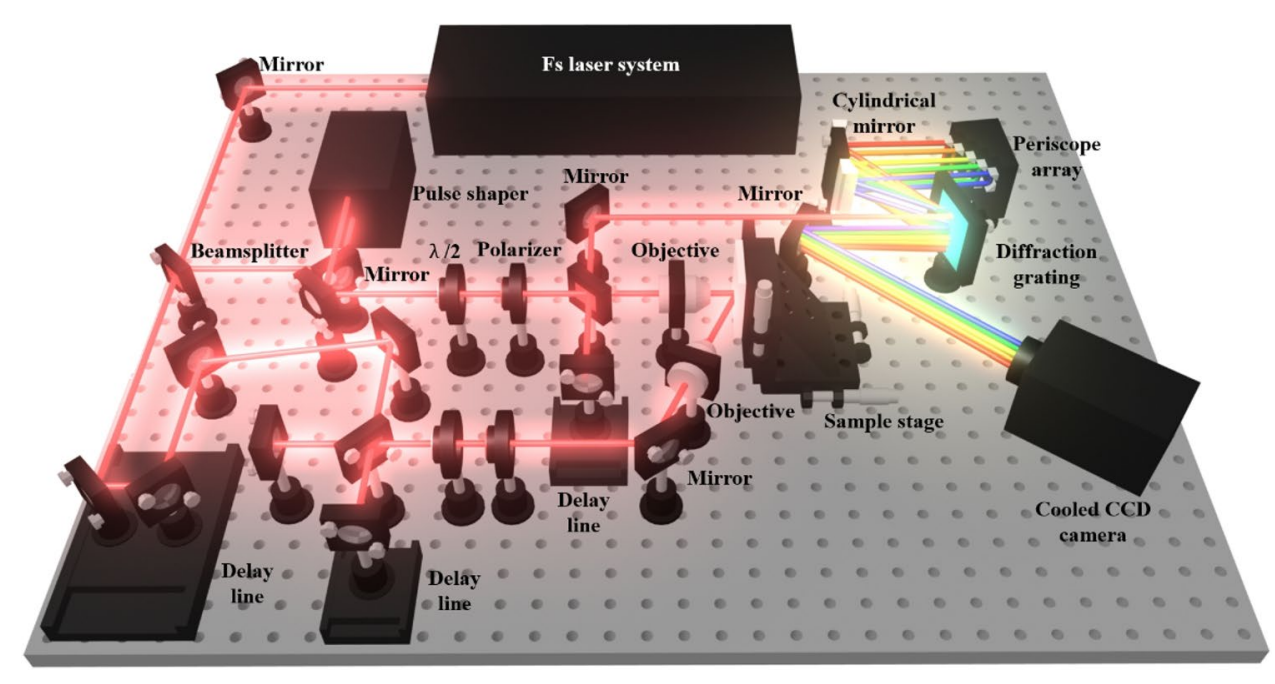




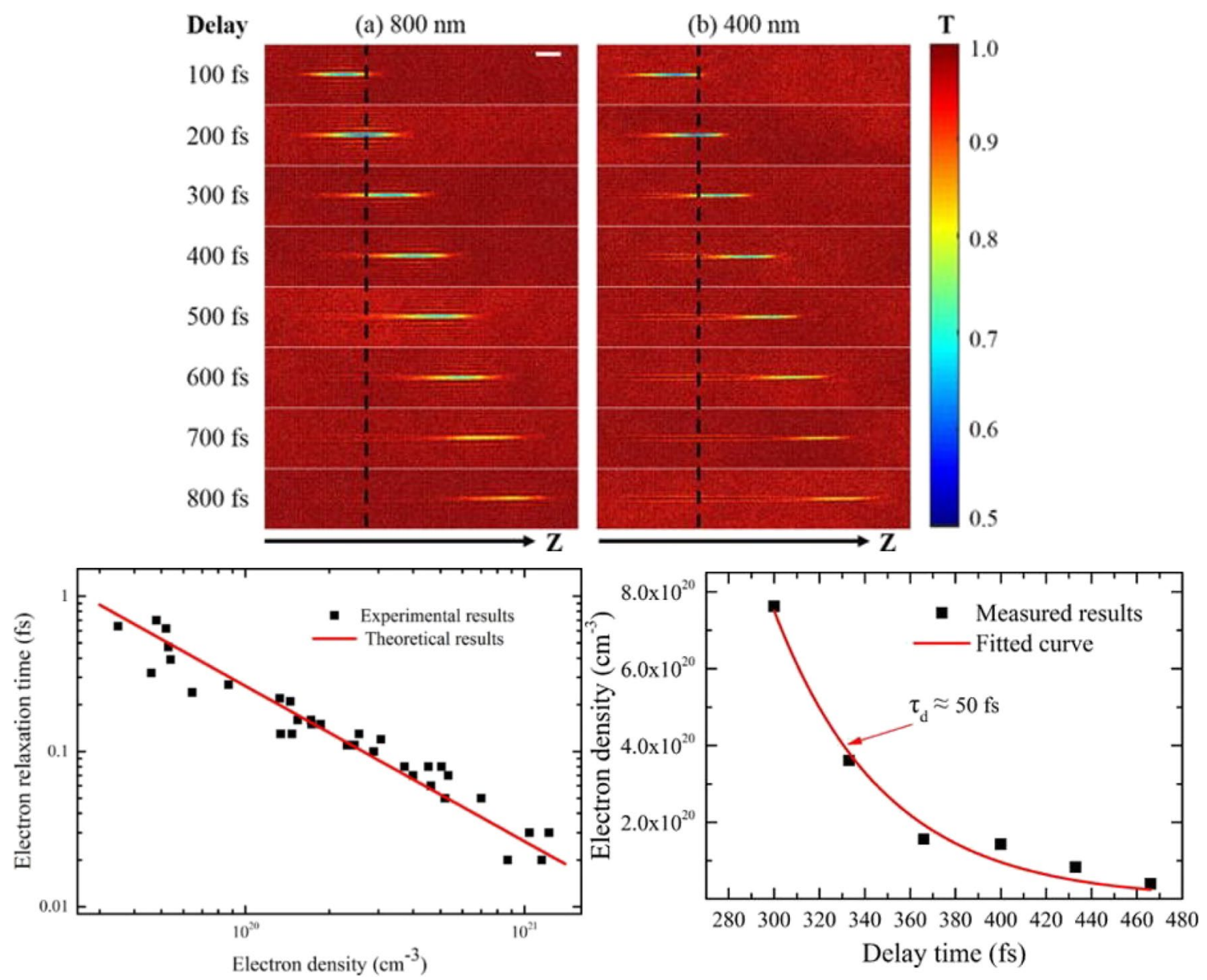

Fig. 9 Time-resolved transmissivities of induced region in fused silica detected using femtosecond laser with a $800-\mathrm{nm}$ and b $400-\mathrm{nm}$ probe pulses at different delay times. The linear relationship between

electron density (c) and electron relaxation time (d) fits well with the theoretical predictions [38]

femtosecond-laser-pulse-coordinated shaping in tempo$\mathrm{ral} / \mathrm{spatial} /$ frequency domain based on electrons dynamics control (Fig. 14).

\subsection{Femtosecond Laser Direct Writing Fabrication}

Femtosecond laser direct writing is the commonly used basic processing method in femtosecond laser manufacturing. Depending on scanning directions, femtosecond laser direct writing can be divided into two methods: horizontal direct writing and vertical direct writing (Fig. 15). In horizontal direct writing, the sample is moved perpendicularly to the laser beam. This method is commonly used to fabricate surface structures $[9,44]$. In vertical direct writing, the sample is moved along the direction of laser beam irradiation. Because the longitudinal depth of the focal spot intensity distribution is generally larger than the transversal dimension, the cross-section of the laser modification zone is asymmetric. To reduce this difference, an objective lens with a larger numerical aperture can be used for tight focusing

or other optical elements can be added to adjust the spatial distribution of pulse intensity [45]. In addition, longitudinal direct writing can be divided into two modes: scanning from the upper surface and scanning from the lower surface [46]. When processing from top to bottom, a femtosecond laser beam is greatly affected by the scattering of ablated materials, which reduces fabrication quality. Yong et al. [47] employed horizontal direct writing to fabricate an ultra-slip surface porous network structure and achieved excellent performance in terms of liquid repellency and cell proliferation resistance(Fig. 16).

\subsection{Femtosecond Fabrication by Laser Pulse Shaping in Time Domain}

Femtosecond laser pulse shaping in the time domain is a new fabrication method that can improve and control ultrafast laser fabrication [49-53]. During femtosecond laser fabrication, most photon energy is initially absorbed by electrons. However, conventional femtosecond laser 

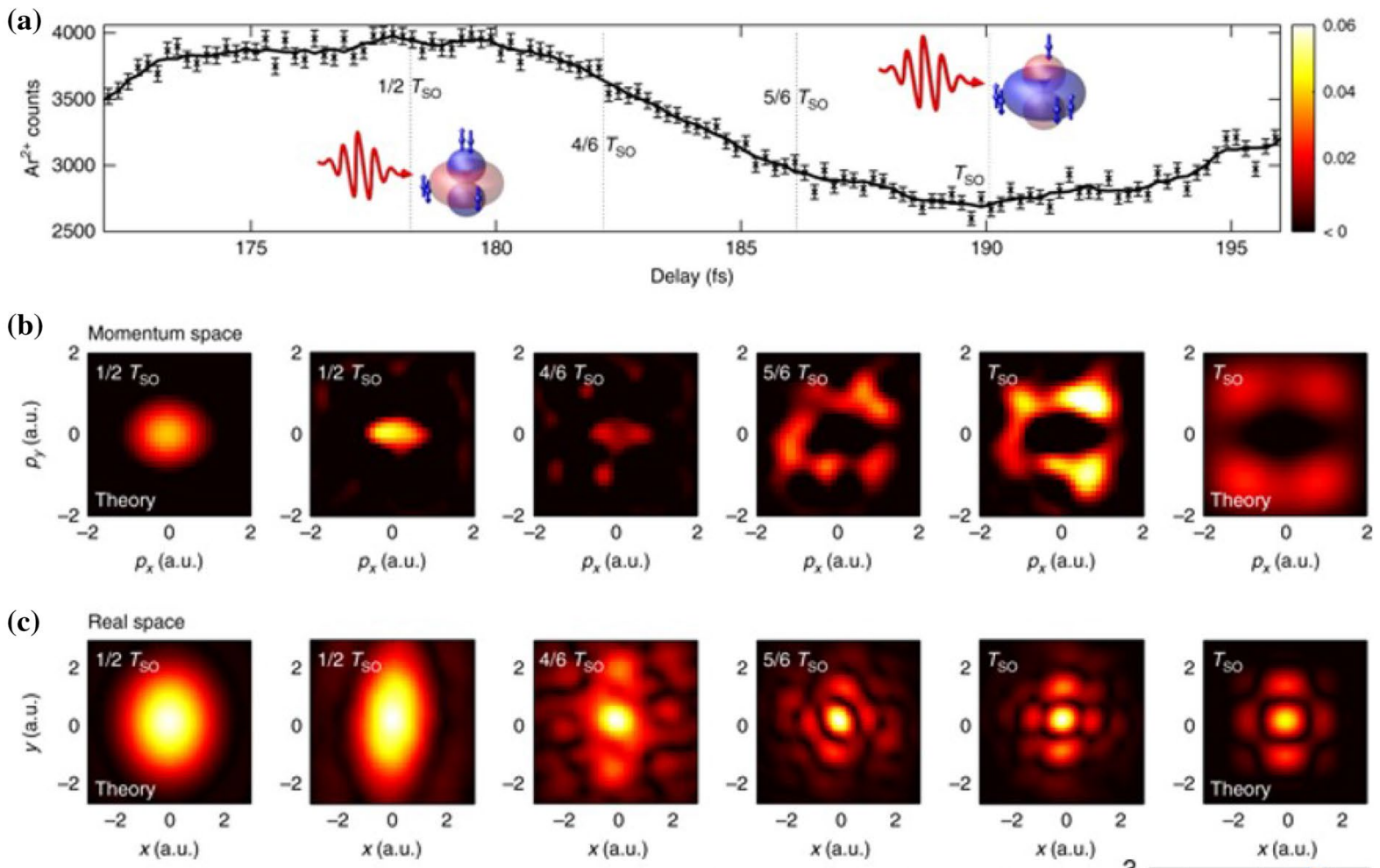
(d) Pump-probe-deflect
scheme

(f) Probe + mid-IR deflection
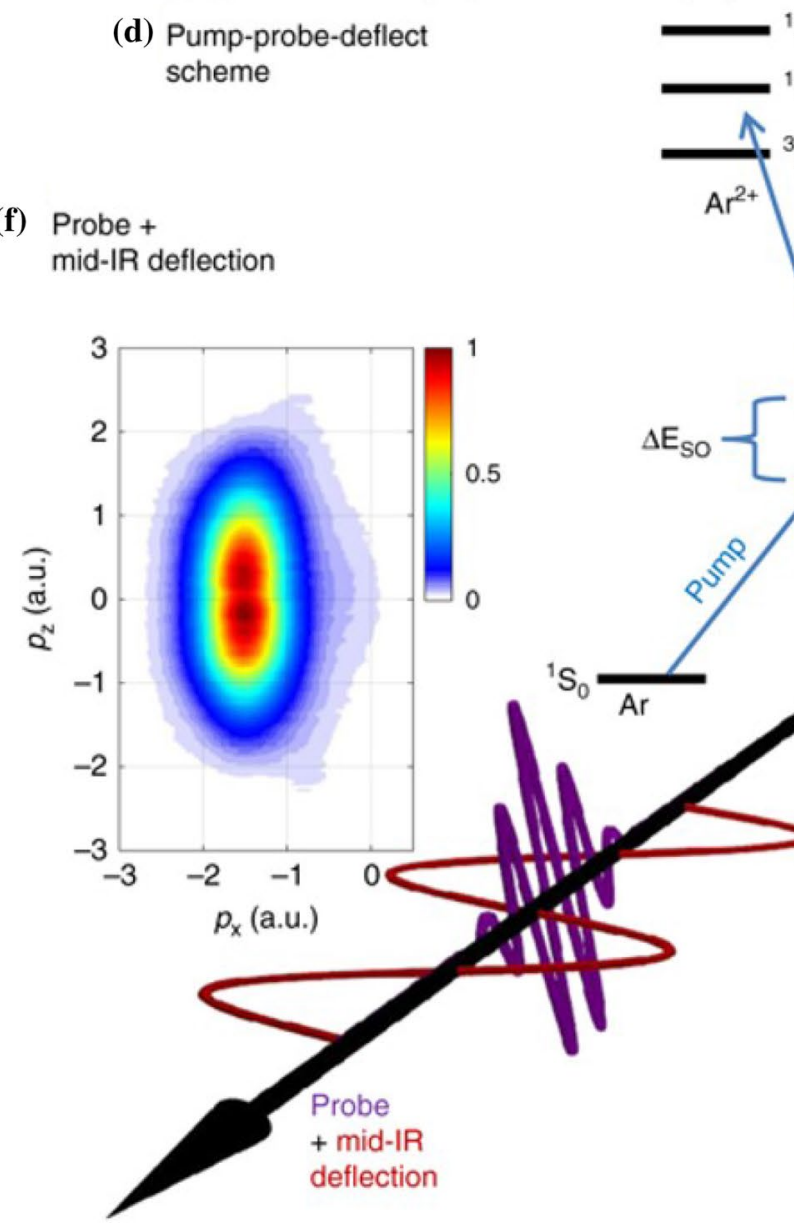

'S (e) Pump only
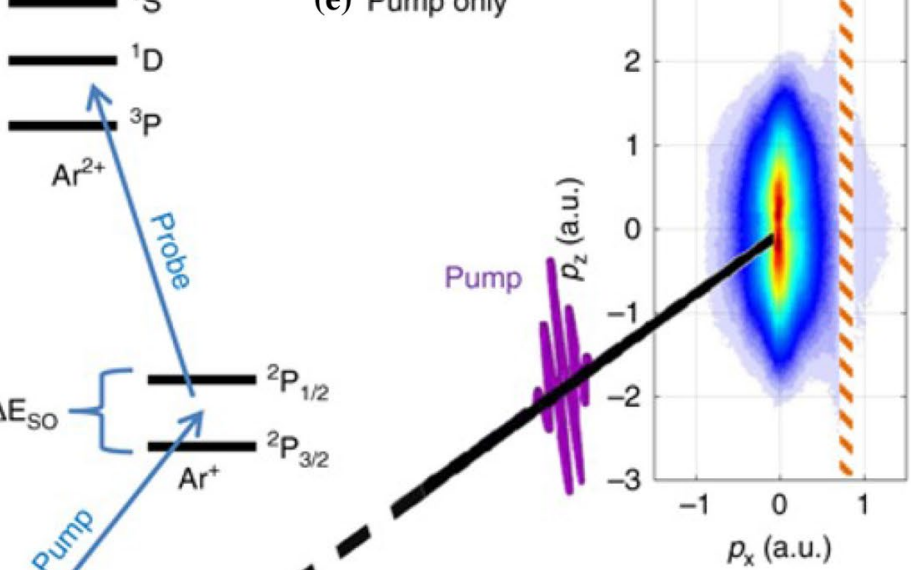

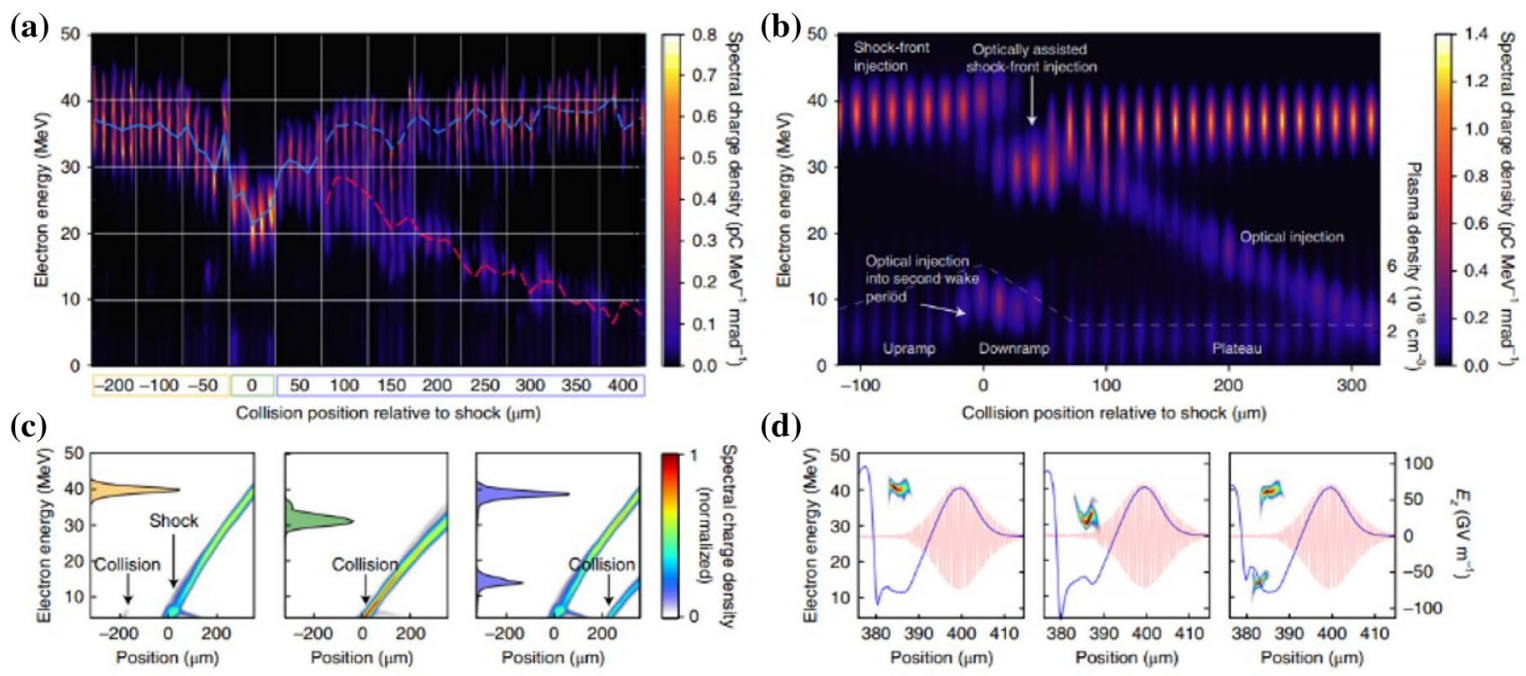

(d)
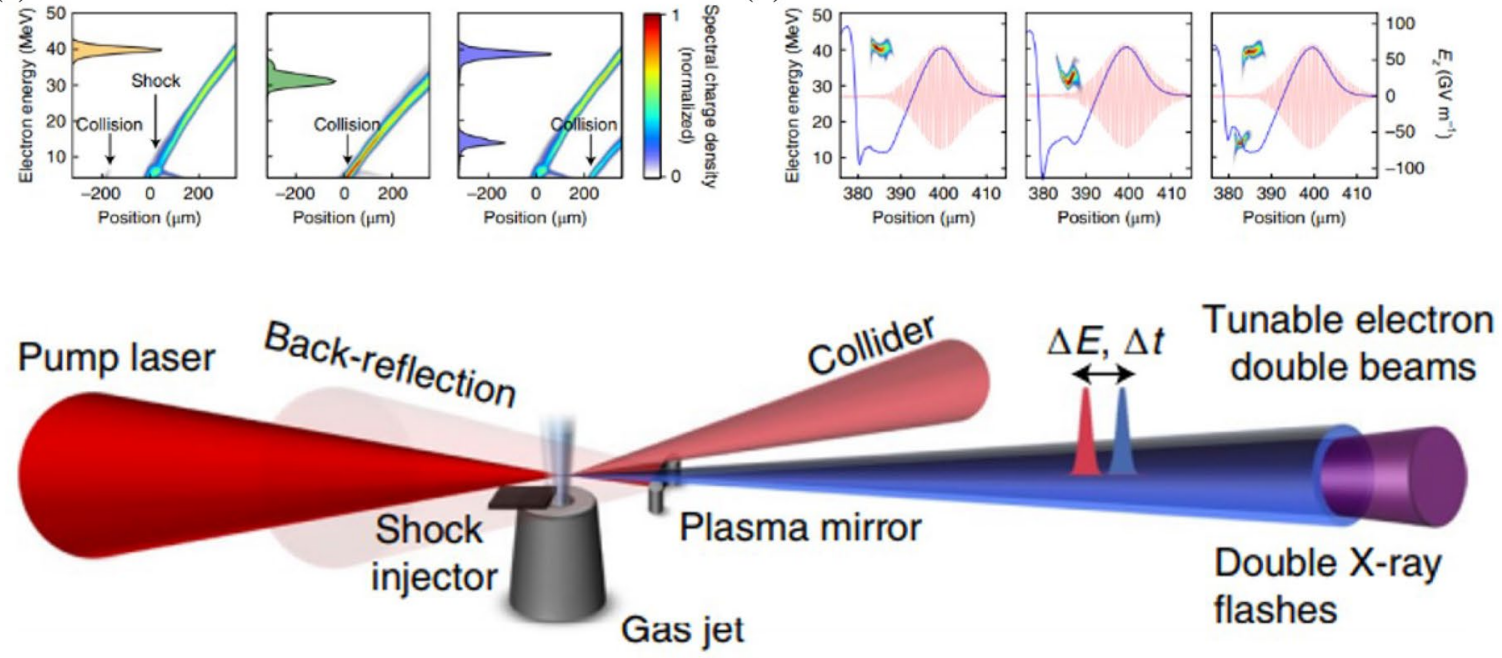

(e)
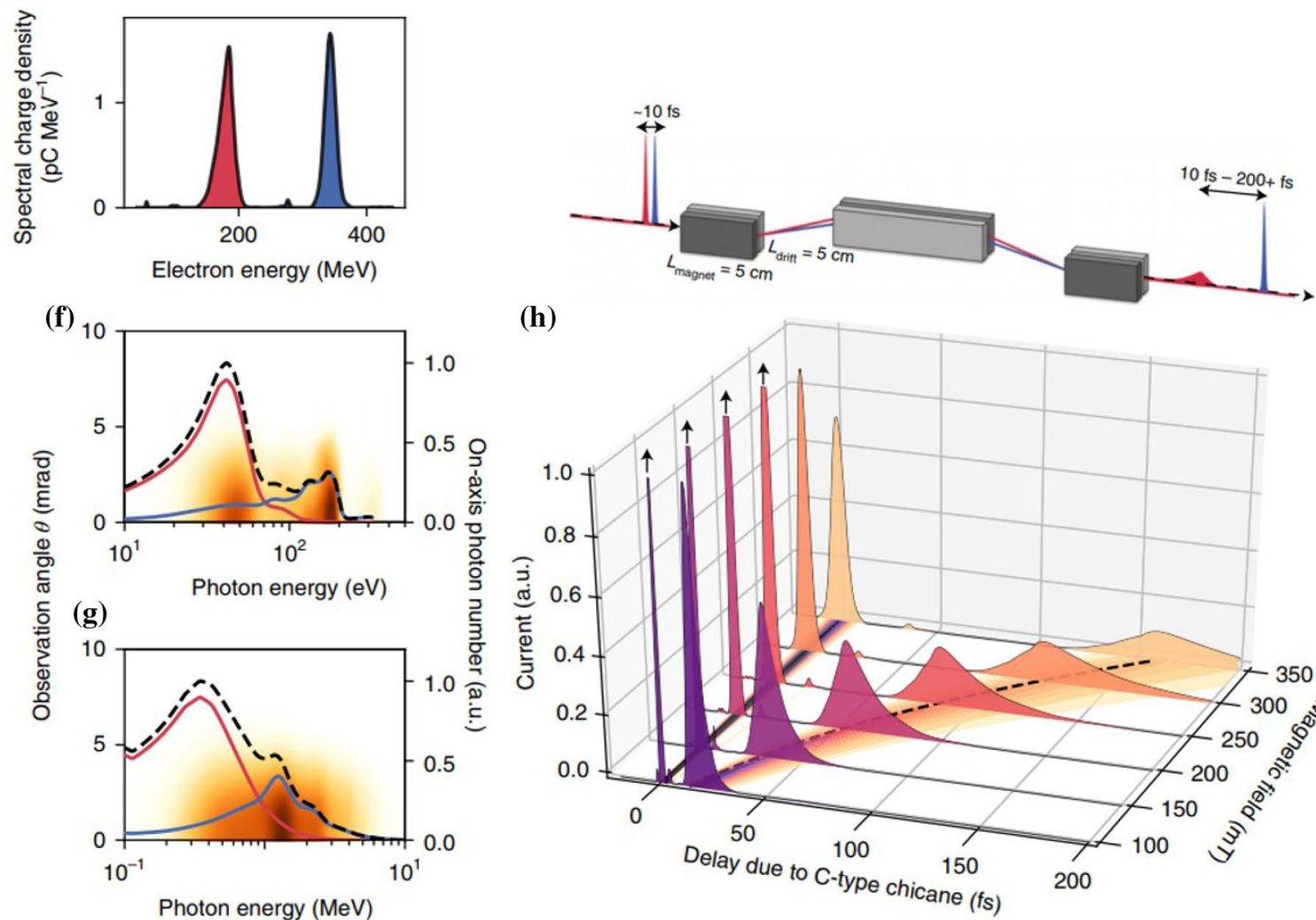

(h)

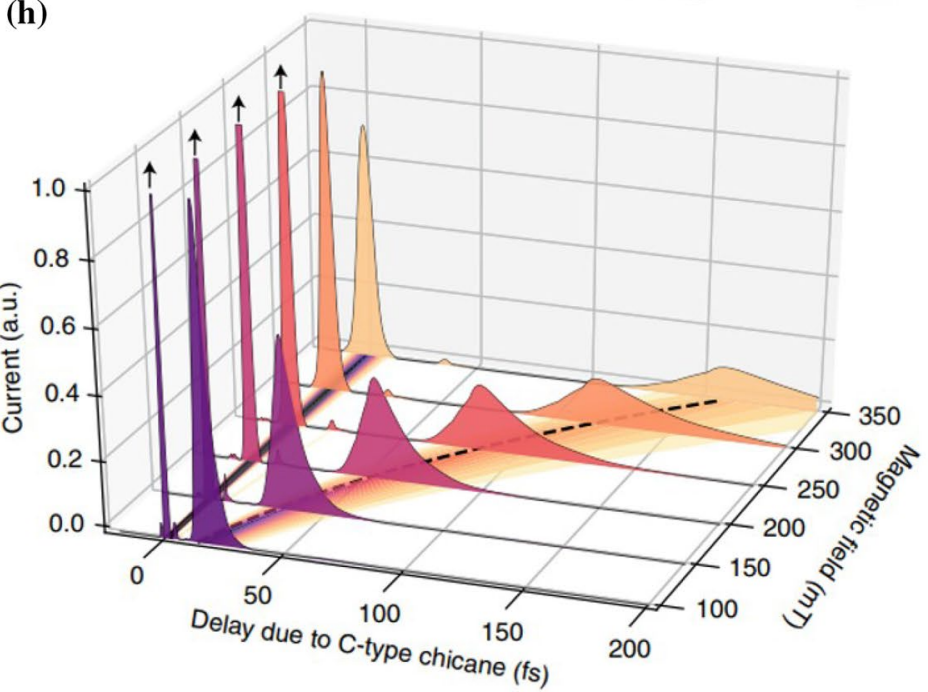

Fig. 11 Demonstration of dual-energy electron beams and PIC simulations. Proposed radiation source and timing control based on dual-energy electron beams [40] 

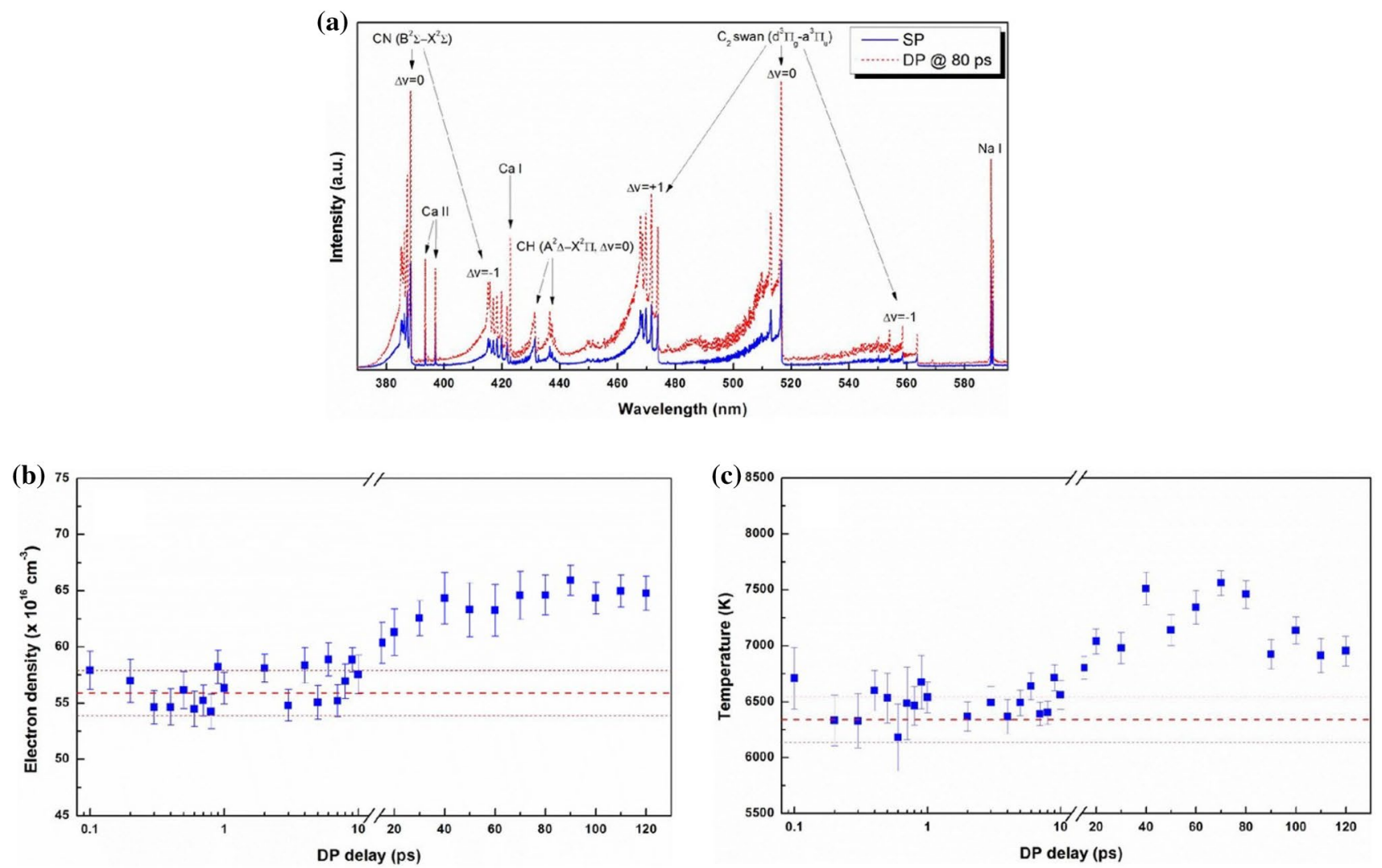

Fig. 12 LIBS of PMMA plasma irradiated by single and double pulses; electron density and temperature of the plasma determined from LIBS are used to characterize laser-induced plasma [41]

Fig. 13 Emission intensities of the spectral peak at $422.66 \mathrm{~nm}$ as a function of the sample temperature for three incident laser fluences $29.7 \mathrm{~J} \mathrm{~cm}^{-2}$ (a), $56.6 \mathrm{~J} \mathrm{~cm}^{-2}$ (b), and $113.2 \mathrm{~J} \mathrm{~cm}^{-2}$ (c). The laser wavelength is $800 \mathrm{~nm}$. Normalized transient reflectivity with the delay time for five different temperatures. The pump laser fluence is $34 \mathrm{~mJ} \mathrm{~cm}^{-2}$ [42]
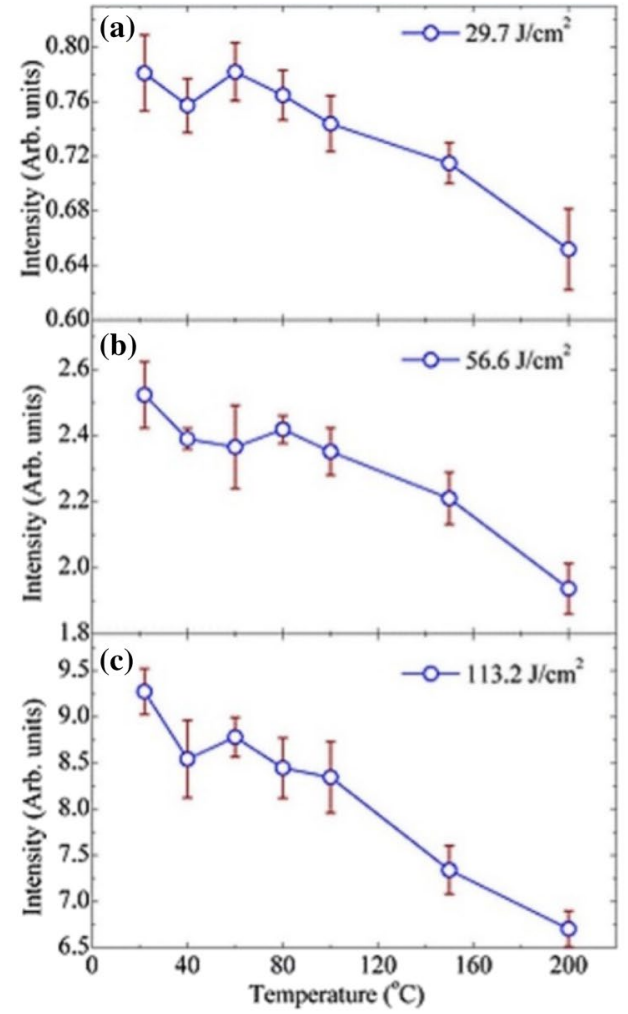

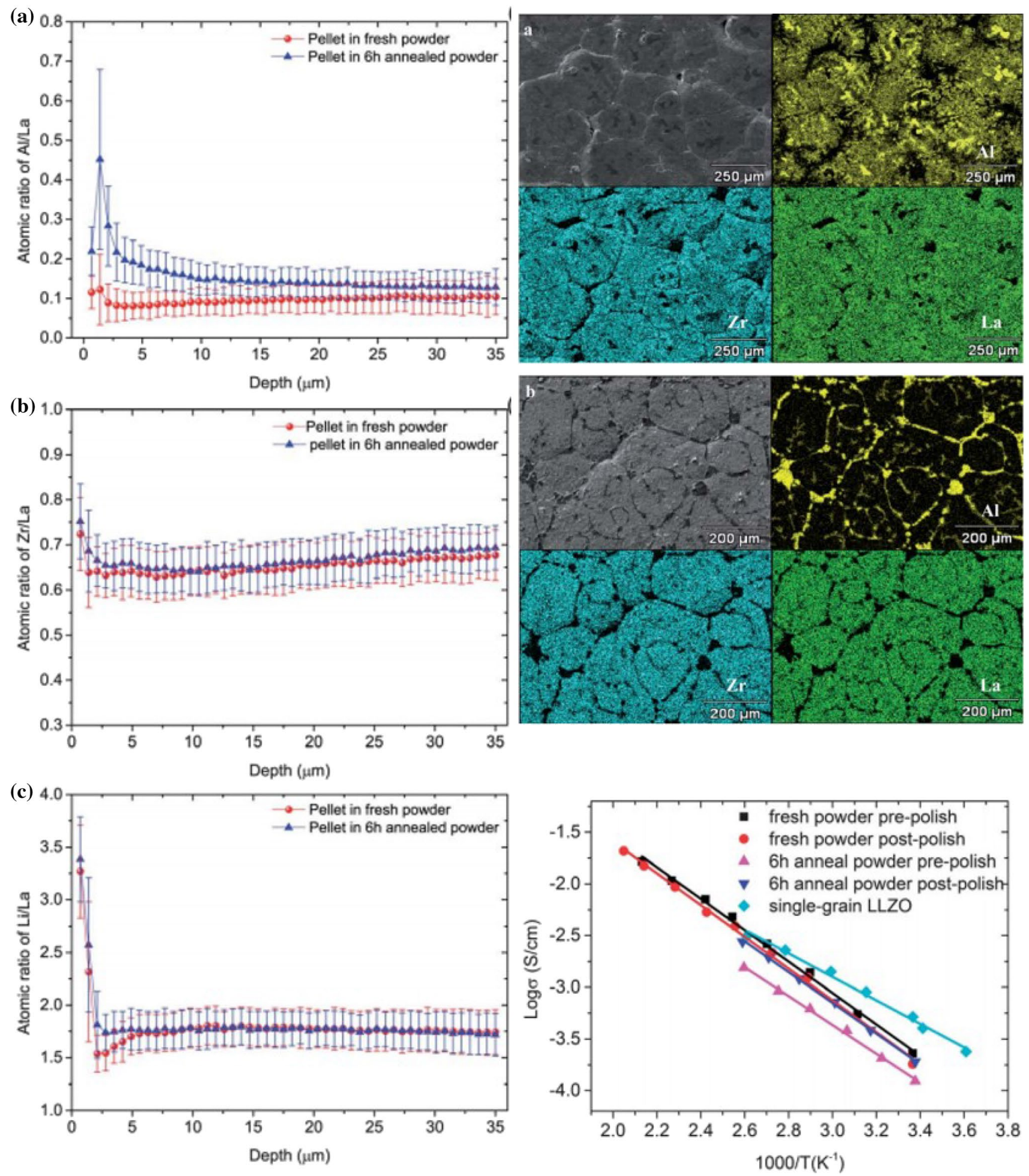

Fig. 14 EDS spectral imaging, LIBS depth profiles, and total ionic conductivities of pellets [43]

pulses are restricted by their repetition rate and are separated by a temporal scale that ranges from microseconds to milliseconds, which is considerably longer than the time scale of electron-lattice coupling (typically in the picosecond range). Temporal pulse shaping facilitates subpulse generation with a pulse delay shorter than the time scale of electron-lattice coupling so that users can control femtosecond laser photon-electron-phonon 

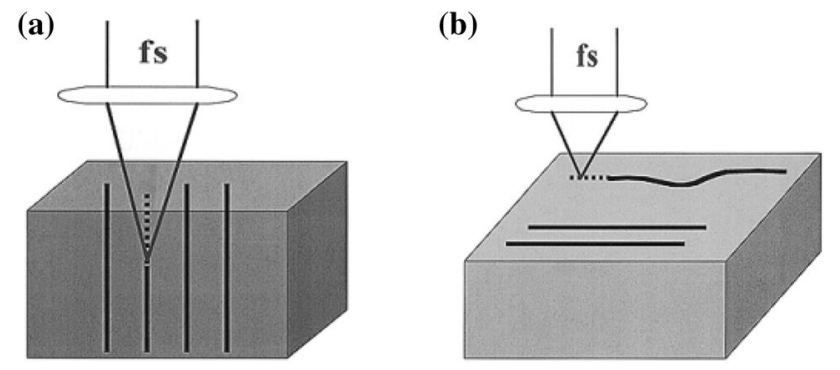

Fig. 15 Laser direct writing geometries: a longitudinal and $\mathbf{b}$ transversal [47]

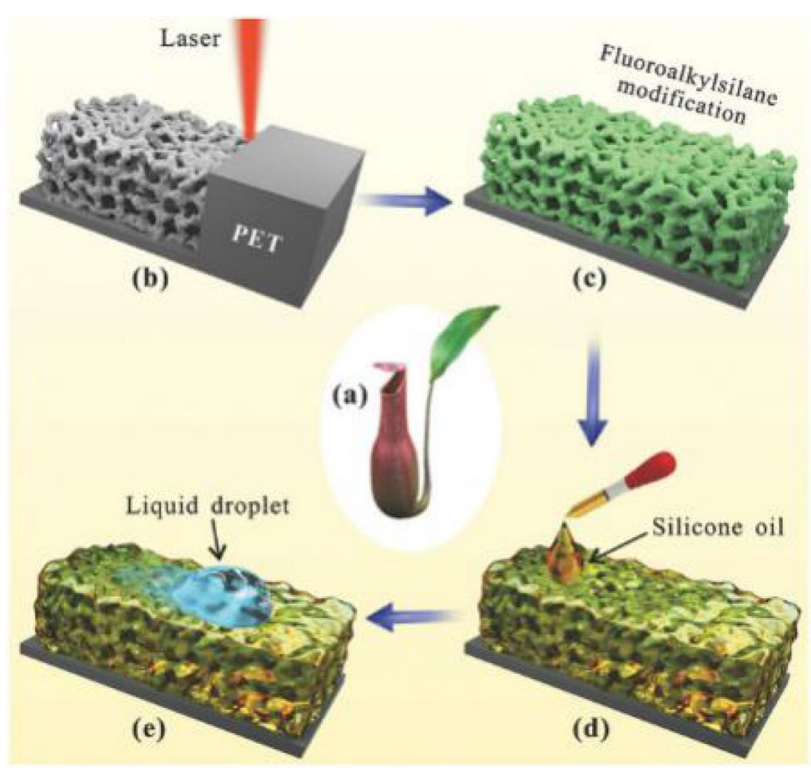

Fig. 16 Schematic of the preparation of a slippery PET surface by femtosecond laser direct writing. a Photo of the Nepenthes pitcher plant. b Femtosecond laser ablation used to generate interconnected porous microstructures. c Use of fluoroalkylsilane modification to lower surface free energy. Green color denotes the fluorosilane molecular layer. d Infusion of the lubricating liquid (silicone oil) into the laser-induced micropores. e Foreign liquid droplet sliding down the as-prepared slippery surface [48]

interactions. The main objective of temporal pulse shaping is the realization of localized transient electron dynamics control through pulse train design to control the physical or chemical properties of processed materials. In general, three main tools are employed to realize temporal pulse shaping: pulse shaper, beam splitter, and thin-film resonator.

\subsubsection{Laser Pulse Shaping Using a Pulse Shaper}

A pulse shaper is a commercially available device that combines Fourier optics and femtosecond laser (Fig. 17) and is used to generate pulse trains to realize Optical Code Division
Multiple Access systems [49], as well as for chemical and biological reaction triggering and monitoring [51]. This device utilizes the Fourier optics principle to fine tune highorder dispersion compensation, perform time-frequency transform of femtosecond laser pulses, obtain pulse spectra with different time distributions, and generate transformlimited few-cycle optical pulses to produce and control pulse trains [52]. Hence, a pulse shaper can be used to shorten or elongate the duration of an optical pulse or to generate complex pulse trains with different subpulses (Fig. 18).

The temporal delay of subpulses can be controlled using a pulse shaper to be shorter than the temporal scale of electron-lattice coupling, which is key for controlling femtosecond laser fabrication. Moreover, a conventional femtosecond single pulse can be shaped into almost any arbitrary pulse train. For example, (1) the pulses can be divided into pulse trains with different numbers of subpulses (Fig. 18b), (2) the delay time between subpulses can be controlled (Fig. 18c), and (3) the energy ratio between subpulses can be controlled (Fig. 18d). These adjusted pulse trains make it possible to directly control photon-electron interactions; control localized transient electron dynamics such as electron density, temperature, and excited state distribution; and modify or optimize the fabrication process.

\subsubsection{Laser Pulse Shaping Using Beam Splitter}

Temporal pulse shaping can be achieved by splitting a laser pulse as well. A beam splitter is generally used to divide an incident pulse into two pulses with a certain intensity ratio and delay time. Hence, a pulse train with two subpulses can be obtained. The delay time between the two subpulses can be controlled through a one-dimensional precision translation stage, and the power ratio can be controlled by a power attenuator. Figure 19 shows a typical fabrication system that uses pulse trains with double subpulses based on the Michelson interference principle. The double subpulses are obtained by splitting the beam once. Similarly, four or more subpulses can be obtained by splitting the beam twice or more times.

\subsubsection{Laser Pulse Shaping Using Thin-Film Resonator}

Another temporal pulse-shaping tool based on a thin-film resonator called a pulse train generator (PTG) was developed recently [54] and is shown in Fig. 20. The PTG comprises two parallel-arranged coating layers-a mirror-coating (MC) layer on a silica film to enhance the interface reflectivity and a beam-splitter-coating (BSC) layer on top of another interface to split the laser pulse. The silica layer between the $\mathrm{MC}$ and the BSC has a predesigned thickness to control the time delay of the subpulses. The formula for calculating the delay time is $2 n \times d / c$, where $n$ is the refractive index of 
Fig. 17 Internal structure diagram of pulse shaper

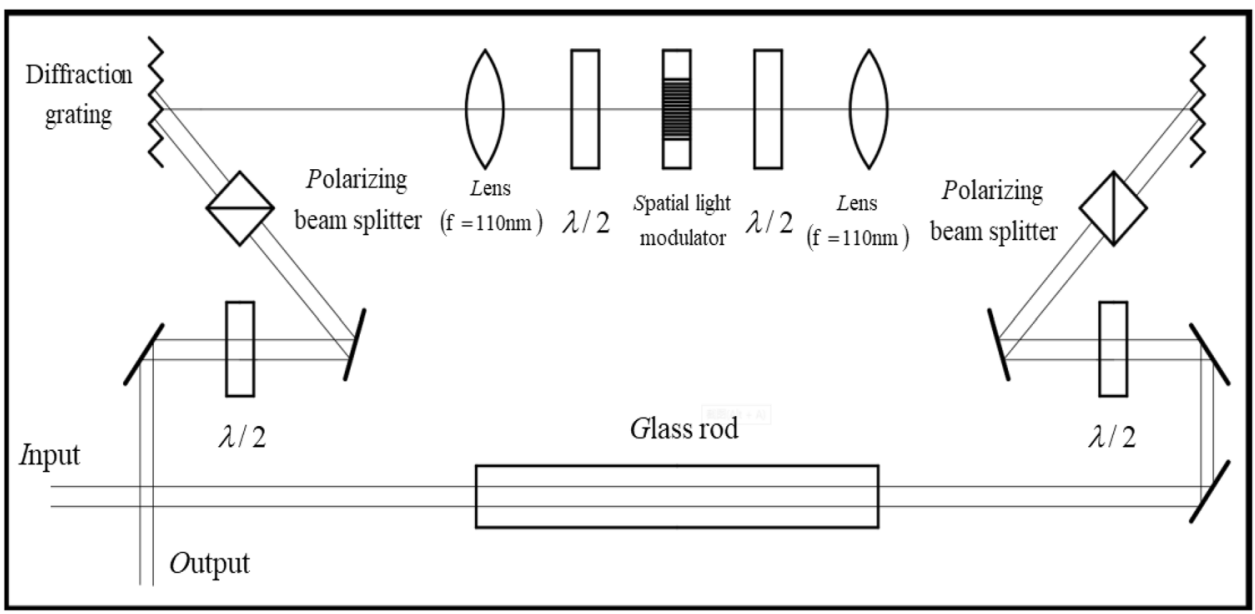

a

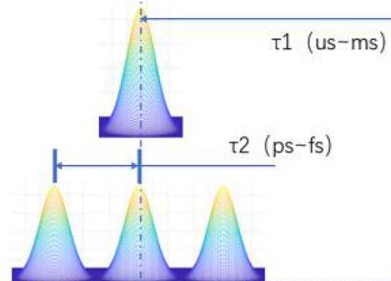

b

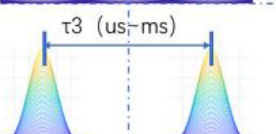

C

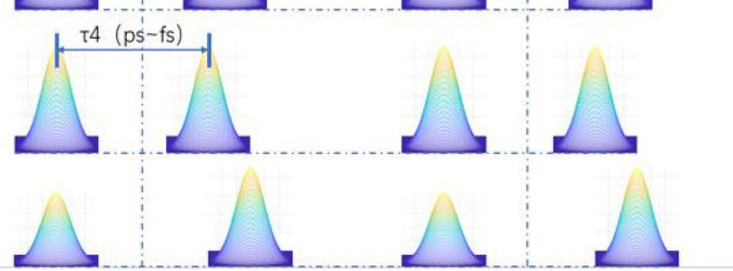

Fig. 18 Femtosecond laser time shaping diagram [53]

the silica coating, $d$ the thickness of the silica layer between the MC and BSC, and $c$ the speed of light. In fact, the silica layer between the MC and BSC is not necessary. The time interval of the generated subpulse sequence can be adjusted directly by the distance (d) between the MC and BSC. When a single pulse is incident on the PTG, the reflected partial energy at the BSC interface generates a first subpulse (SP-1). The remaining pulse energy is passed through and reflected back at the MC interface. The reflected pulse is separated by the BSC again to generate a second subpulse (SP-2). Other subpulses are then generated in a similar manner. Intensity calculation of the subpulses obtained from the PTG is summarized in Table 2.

Here, $E$ is the intensity of the incident pulse, $s$ the reflecting ratio of the BSC, and $r$ the reflectivity of the MC. Figure 20 shows a schematic of the PTG and the experimental setup. In this experiment, the splitting ratio of the BSC is

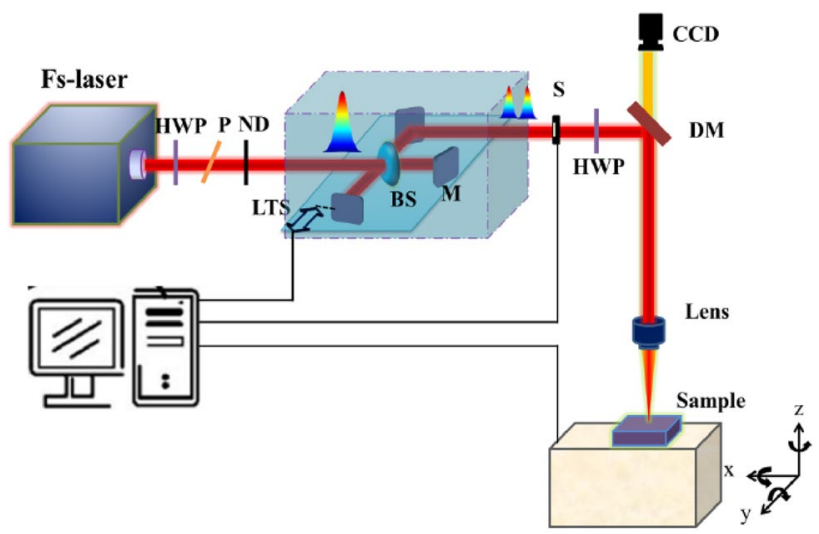

Hexapod 6-axis positioning system

Fig. 19 Time-domain shaping femtosecond laser fabrication system based on Michelson's interference principle. $H W P$ half-wave plate, $P$ polarizer, $N D$ neutral density attenuator, $B S$ beam splitter, $M$ reflector, LTS linear mobile platform, $S$ shutter, $D M$ dichroic mirror, and $C C D$ charge-coupled device

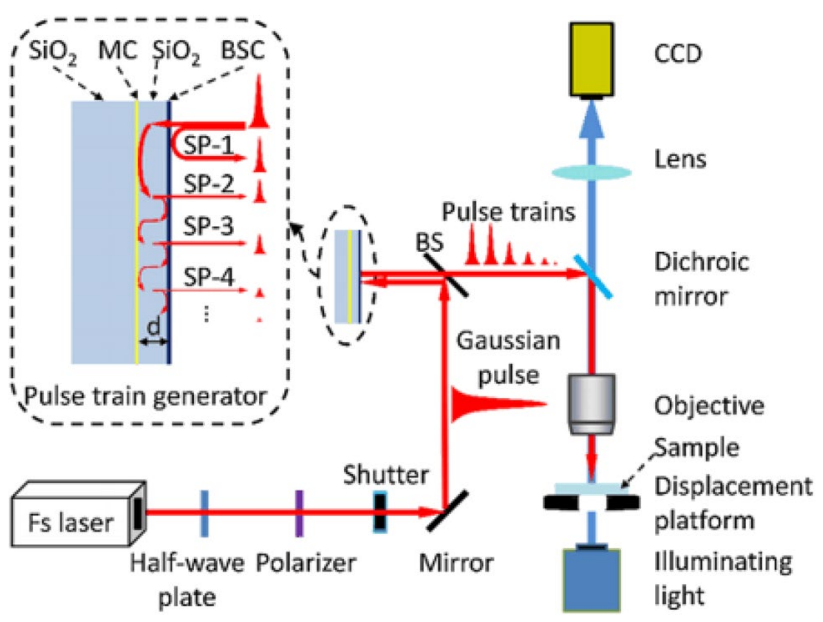

Fig. 20 Schematic of the PTG and experimental setup: $M C$ mirror coating, $B S C$ beam splitter coating, $S P$ subpulse, $d$ distance between $\mathrm{BSC}$ and $\mathrm{MC}$, and $B S$ beam splitter [55] 
Table 2 Subpulse intensity from thin-film pulse train generator

\begin{tabular}{ll}
\hline Sub-pulse & Intensity \\
\hline SP-1 & $s * E$ \\
SP-2 & $(1-s)^{2} * r^{*} E$ \\
SP-3 & $s^{*}(1-s)^{2} * r^{2} * E$ \\
SP-4 & $s^{2 *}(1-s)^{2 *} r^{3 *} E^{*}$ \\
$\ldots$ & $\ldots$ \\
SP- $n$ & $s^{n-2} *(1-s)^{2} * r^{n-1} * E$ \\
\hline
\end{tabular}

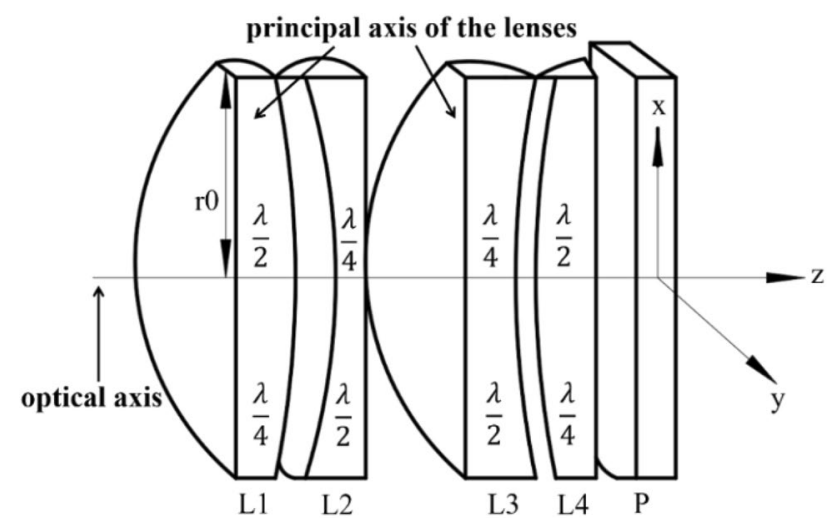

Fig. 21 Schematic of spatial light shaping of birefringent lens group

4:6, $40 \%$ reflected. Hence, the intensity ratio of the first seven subpulses is approximately 0.400:0.360:0.144:0.06: 0.023:0.009:0.004.

\subsection{Laser Pulse Shaping in Space Domain}

In addition to temporal pulse shaping, another key method for controlling laser fabrication is pulse shaping in the spatial domain. The spatial shaping technique modulates the waveform of a femtosecond laser into an arbitrary shape, which control the properties and effects of the processed materials.

\subsubsection{Double-Refractive Lens Group for Laser Pulse Spatial Shaping}

To improve the overall efficiency of a femtosecond laser system and fully utilize its energy, it is necessary to shape the Gaussian beam into a flat-top beam with a uniform spatial distribution, which can be achieved using a group of birefringent lenses (Fig. 21). The system consists of two pairs of birefringent crystal lenses-L1, L2, L3, L4-and an analyzer $\mathrm{P}$. The birefringent lens group is flexible and convenient for spatial beam shaping and is especially suitable for linearly polarized Gaussian beams. It can flexibly change its transmittance function as the beam parameters change to form a flat-top beam, as shown in Fig. 22b, c.

\subsubsection{Liquid Crystal Spatial Light Modulator for Spatial Pulse Shaping}

The liquid crystal spatial light modulator (SLM) is a laser beam spatial shaping system composed of an electrical writing liquid crystal. Electrically addressed liquid crystal SLM is conveniently interfaced with a computer to modulate the amplitude or phase of the corresponding unit pixel in a set optical modulation mode. It can modulate a Gaussian beam into a beam with different spatial distributions such as a square flat-top beam, circular flat-top beam, vortex beam, or Bessel beam depending on fabrication requirements (Fig. 22), and improve the fabrication quality and accuracy. The optical path diagram of a spatial light shaping fabrication system based on SLM is shown in Fig. 23.
Fig. 22 Spatial light shaping results. a Gaussian beam; b square flat-top beam; $\mathbf{c}$ circular flat-top beam; d vortex beam; $\mathbf{e}$ Bessel beam

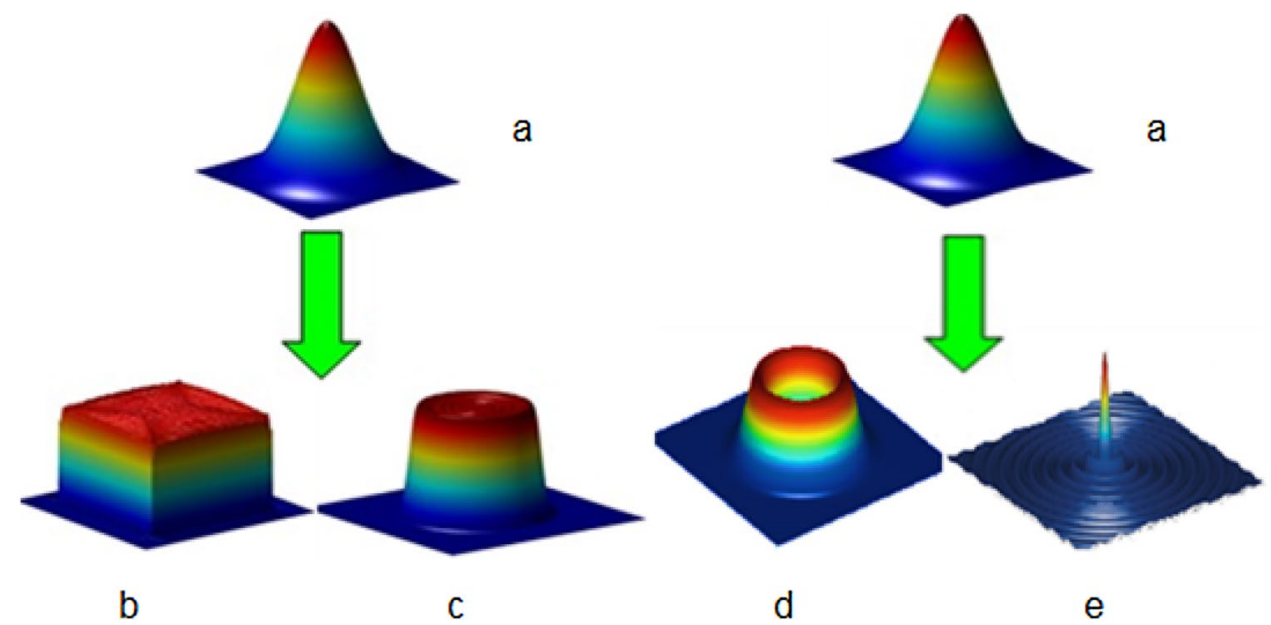

Springer 


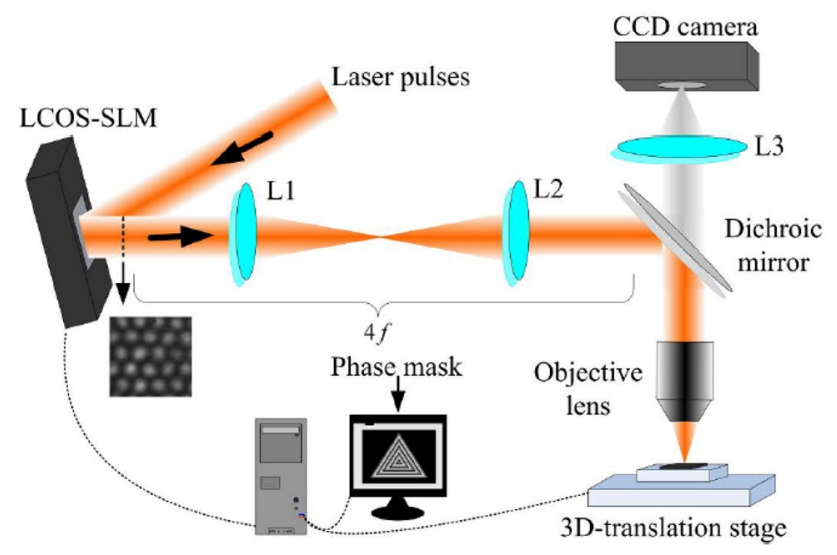

Fig. 23 Optical path diagram of spatial pulse shaping fabrication system based on liquid crystal SLM

\subsection{Femtosecond Fabrication by Laser Pulse Shaping in Frequency Domain}

Temporal and spatial pulse shaping are two major methods for controlling the fabrication process based on the electron dynamics control. In addition to the temporal and spatial attributes, another critical parameter of femtosecond laser is the frequency, which can also affect the fabrication process. Therefore, another method of femtosecond laser fabrication based on the laser frequency tuning through nonlinear optical effects, such as second harmonic generation and optical parametric amplification, is proposed. Although the related research results are few, it is still a potential research direction.

Second harmonic generation is a well-known nonlinear optical effect that can double the laser frequency. As shown in Fig. 24, after a laser pulse with a frequency of $\omega_{1}$ incident on a nonlinear crystal, another laser pulse with a frequency of $\omega_{2}\left(\omega_{2}=2 \omega_{1}\right)$ will be generated.

Compared with the second harmonic generation, optical parametric amplification (OPA) has several advantages, such as large range of wavelength tunability, and high gain $\left(10^{3}-10^{9}\right)$. Usually, the wavelength tuning range of an OPA can reach $0.45-2.6 \mu \mathrm{m}$ [55]. In the mid-infrared range, $\mathrm{KTiOPO}_{4}$ crystals can be used as a working medium to generate tunable wavelength pulses from $2.5 \mu \mathrm{m}$ to $4.4 \mu \mathrm{m}$ [56], which is of great significance for studying the vibration energy levels of molecules. Moreover, on the basis of the advantage of large gain bandwidth, high-energy periodicorder optical pulses or femtosecond pulses can be obtained using super continuous white light as the injection source. The former is indispensable for studying physical and chemical reactions, as well as ultrafast phenomena of organisms, and the latter has great practical significance in inertially constrained fast ignition devices [57].

Figure 25 shows a typical OPA process. When a strong laser with a frequency of $\omega_{\mathrm{p}}$ (pump light) is incident on a nonlinear medium, a signal light with a frequency of $\omega_{\mathrm{s}}$ and an idle light with a frequency of $\omega_{\mathrm{i}}\left(\omega_{\mathrm{p}}=\omega_{\mathrm{s}}+\omega_{\mathrm{i}}\right)$ are generated. Then, the single beam $\left(\omega_{\mathrm{s}}\right)$ is amplified to continue the fabrication process. Therefore, the laser frequency $\omega_{\mathrm{p}}$ can be tuned over a large range of signal frequency $\omega_{\mathrm{s}}$ according to the fabrication requirements.

\subsection{Femtosecond Laser Chemical Fabrication}

Professor Zewail began using a femtosecond laser to study the chemical reaction process in 1980, thus opening up the entirely new domain of femtosecond chemistry [58]. In fact, by shaping ultrafast laser pulses in the time/space/frequency

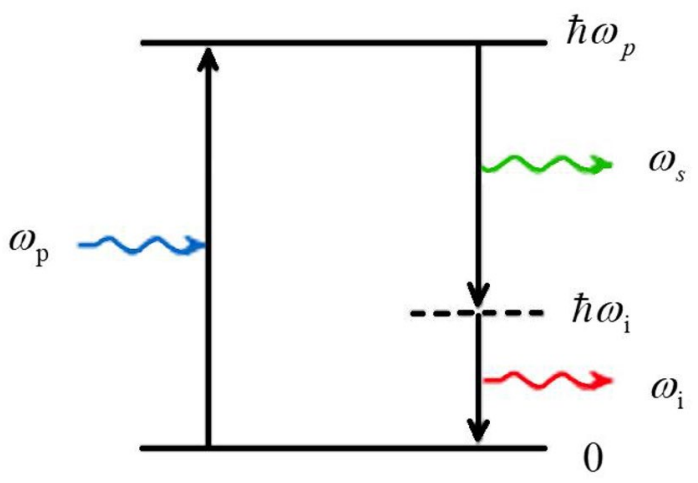

Fig. 25 Photon picture of OPA: A pump photon excites a virtual energy level whose decay is stimulated by a signal photon, resulting in the emission of an identical second signal photon and an idler photon under energy and momentum conversion

Fig. 24 Diagram of second harmonic generation

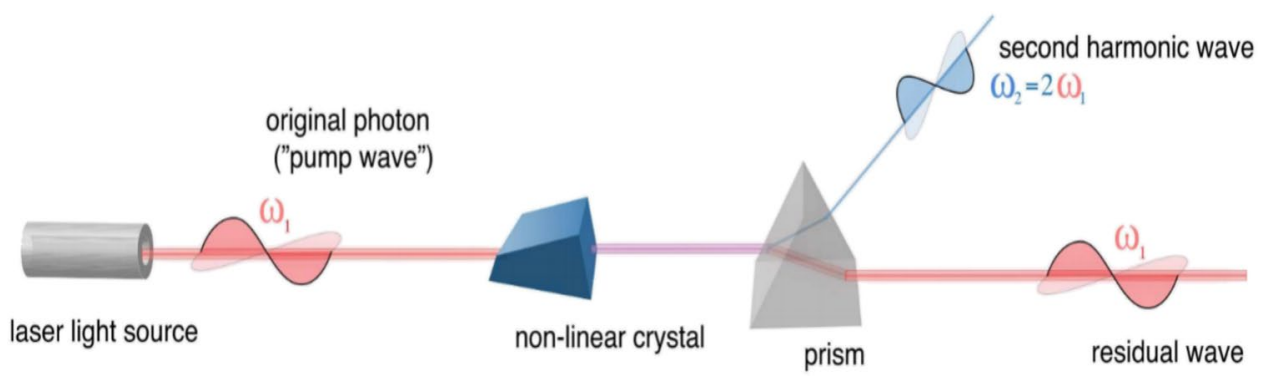


domain, transient local electron dynamics (electron density, temperature, and distribution) of materials can be controlled, thus helping control chemical reaction processes such as chemical bond cleavage; new bond formation; proton and electron transfer; compound isomerization; molecular dissociation; reaction intermediates; final product speed, angle, and state distribution; chemical reactions in solution and solvent action; effects of vibration and rotation in the molecule on chemical reactions; and photochemical reactions. This section mainly introduces femtosecond laser photoreduction fabrication, femtosecond laser photopolymerization fabrication, and femtosecond laser photoisomerization.

\subsubsection{Femtosecond Laser Photoreduction Fabrication}

Photoreduction is a common chemical reaction process. For example, photosynthesis in plant chloroplasts is a typical photoreduction reaction. Conventional high-resolution metal structure deposition methods require high-vacuum equipment, complex cover steps, and corrosive chemical solutions as well as expensive masks $[59,60]$. The femtosecond laser photoreduction process does not have these limitations because it uses high-energy irradiation from a femtosecond laser to excite electrons in a metal solution and electrons are reduced to metal atoms by normal-valent metal ions, thereby forming metal micro/nano structures. For example, Seiya et al. [61] achieved the generation of metallic silver regions in thin silver-containing films deposited on glass substrates by photoreduction of silver ions induced by multiphoton absorption of closely focused femtosecond laser pulses (Fig. 26).

\subsubsection{Femtosecond Laser Photopolymerization Fabrication}

In femtosecond laser photopolymerization, a few photosensitive organic substances, such as photosensitive resins, undergo a chain reaction after absorbing photons to form a polymer. Through this process, nonlinear absorption of the femtosecond laser by the bonding material helps process nanostructures. Recently, Pedro et al. [62] used femtosecond laser two-photon polymerization to process acrylate polymers and realized nanostructures fabrication (Fig. 27).

\subsubsection{Femtosecond Laser Photoisomerization}

Photoisomerization refers to the structural transformation that occurs between certain isomers under photoexcitation. Structural changes in organic materials lead to changes in physical and chemical properties, such as solubility and chemical activity. Under irradiation by ultraviolet light or near-infrared light, the double bond of DPO-PPV rotates and the molecular structure changes from the original twisted structure to the coplanar structure; that is, from the

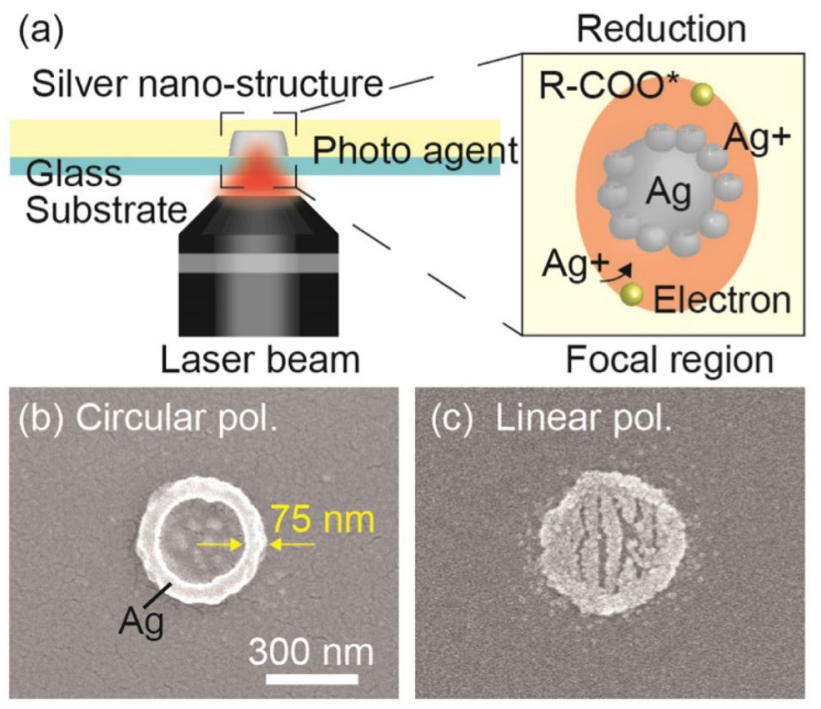

Fig. 26 a Laser fabrication of silver nano-rings and mechanism of photoreduction, b SEM image of the nano-ring fabricated using circularly polarized laser beam, c SEM image of an area fabricated using linearly-polarized beam at the same exposure conditions as used for the nano-ring in $\mathbf{b}$ [61]

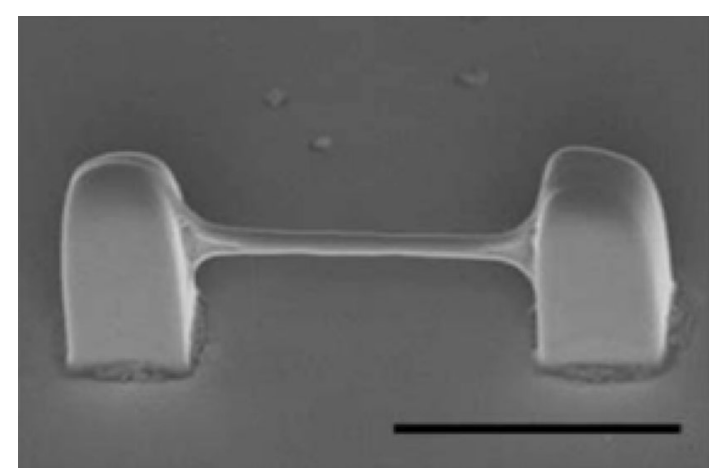

Fig. 27 The femtosecond laser uses an SEM image of the microstructure of the photopolymerization process. The scale bar is $10 \mu \mathrm{m}$ [62]

cis-structure to the trans-structure [63]. Because the transstructured molecules are coplanar structures, attraction between the molecules is greatly enhanced and solubility in the organic solution is lowered. Therefore, DPO-PPV precipitates under laser irradiation and micro/nano fabrication is finally achieved.

Zhao et al. [64] found that the inactive six-membered ring structure of the $\mathrm{Si}-\mathrm{O}$ bond in fused silica under femtosecond laser irradiation is transformed into an active three-membered ring and a four-membered ring structure (Fig. 28). The hydrofluoric acid etching efficiency of the fused silica surface after time-shaping femtosecond laser double-pulse irradiation was 37 times higher than that after a single pulse. 


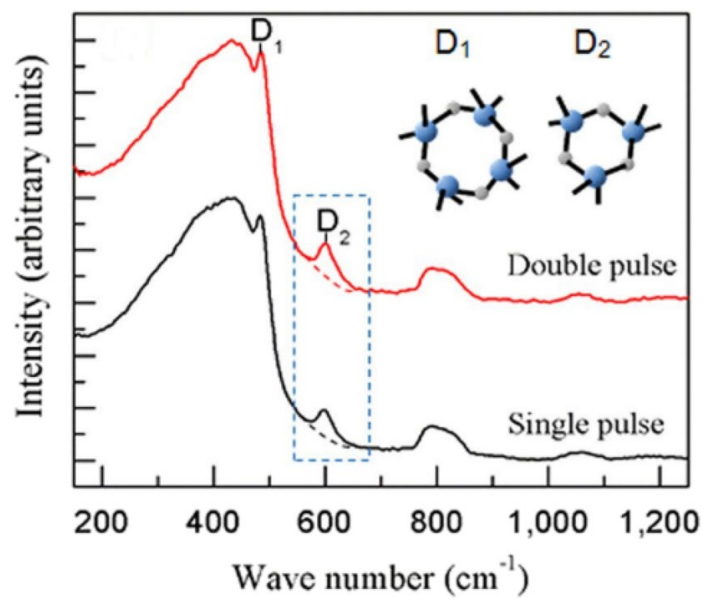

Fig. 28 Femtosecond laser photoinduced fused silica isomerism [64]

Different femtosecond laser fabrication methods described above are summarized in Table 3.

\section{Applications}

With the progress of theories and methods, many applications of femtosecond laser micro/nano fabrication have been developed in the following frontier areas: different functional surfaces and materials, laser surgery, cell transfection, high-aspect-ratio microholes, and laser shock peening. The technique offers considerable advantages over traditional fabrication methods.

\subsection{Femtosecond Laser for Constructing Special Wetting Surfaces}

Wettability is a basic property of solid material surfaces. Regulation of surface wettability is of great significance from the viewpoint of applying materials in biomedical, bionic, paint, lubrication, liquid transportation, selfcleaning, and many other fields. Therefore, research on superhydrophobic, super-thin oil, super-double-sparse, super-hydrophilic, and other extreme wettability surfaces has received extensive attention in recent years.

\subsubsection{Superhydrophobic Surfaces}

Superhydrophobic surfaces have a wide range of applications in many areas such as self-cleaning $[65,66]$, oil-water separation [67-69], anti-icing [70, 71], and drag reduction $[72,73]$. Because the superhydrophobic properties of a substance depend mainly on the surface topography and chemical composition of a material $[74,75]$, femtosecond lasers offer two major advantages in this respect compared with conventional methods: (1) strong controllability, the method can be used to precisely control the microscopic topography of a material surface; (2) multi-material adaptability, the technique can be applied to almost any materials such as silicon, polydimethylsiloxane (PDMS), glass, grapheme, copper, titanium, and zinc.

Table 3 Summary of different femtosecond laser fabrication methods

\begin{tabular}{|c|c|c|}
\hline Laser fabrication methods & Method of implementation & Advantages \\
\hline Femtosecond laser direct writing fabrication & Direct scanning & Fast and simple \\
\hline \multirow[t]{3}{*}{$\begin{array}{l}\text { Femtosecond fabrication by laser pulse shap- } \\
\text { ing in time domain }\end{array}$} & (1) Laser pulse shaping using a pulse shaper & $\begin{array}{l}\text { Phase and amplitude modulated pulse can be } \\
\text { implemented simultaneously }\end{array}$ \\
\hline & (2) Laser pulse shaping using beam splitter & Optical path is easy to implement and flexible \\
\hline & $\begin{array}{l}\text { (3) Laser pulse shaping using thin-film resona- } \\
\text { tor }\end{array}$ & Low cost and compact \\
\hline \multirow[t]{2}{*}{ Laser pulse shaping in space domain } & $\begin{array}{l}\text { (1) Double-refractive lens group for laser pulse } \\
\text { spatial shaping }\end{array}$ & Easy to operate and convenient \\
\hline & $\begin{array}{l}\text { (2) Liquid crystal spatial light modulator for } \\
\text { spatial pulse shaping }\end{array}$ & Optical field shaping is flexible \\
\hline \multirow{2}{*}{$\begin{array}{l}\text { Femtosecond fabrication by laser pulse shap- } \\
\text { ing in frequency domain }\end{array}$} & (1) Second harmonic generation & Simple and low cost \\
\hline & (2) Optical parametric amplification (OPA) & $\begin{array}{l}\text { Adjustable wavelengths, and large gain band- } \\
\text { width }\end{array}$ \\
\hline \multirow[t]{3}{*}{ Femtosecond laser chemical fabrication } & $\begin{array}{l}\text { (1) Femtosecond laser photoreduction fabrica- } \\
\text { tion }\end{array}$ & $\begin{array}{l}\text { Convenient for integrated manufacturing of } \\
\text { different chemical components }\end{array}$ \\
\hline & $\begin{array}{l}\text { (2) Femtosecond laser photopolymerization } \\
\text { fabrication }\end{array}$ & High resolution of far beyond diffraction limit \\
\hline & (3) Femtosecond laser photoisomerization & $\begin{array}{l}\text { Suitable for inducing structural transformation } \\
\text { between certain isomers }\end{array}$ \\
\hline
\end{tabular}


The surface of the lotus leaf exhibits superhydrophobic properties owing to the micro/nano composite structures on the surface [76]. The study found that two main factors affect the surface hydrophilic/hydrophobic properties of solid materials. One is the free energy of the material surface and the other is the surface roughness of the material [77-82]. The Wenzel model holds that water droplets are always filled with microgrooves on a solid surface. These grooves increase the contact area between water and the surface, amplify the hydrophobic effect in geometry, and increase the contact angle (CA) of water droplets [78, 79]. The Cassie model holds that the contact of a droplet on a rough surface is a composite contact of solid-gas-liquid. The nanostructure covered by the rough surface has a residual air layer. Water droplets fall on air and are not in adequate contact with the material to attain hydrophobicity [80], [81]. The contact of water droplets with the solid surface in different states is shown in Fig. 29.

Silicon is a typical intrinsically hydrophilic material. Zorba et al. [83] prepared a superhydrophobic structure on a silicon surface by using a femtosecond laser in $\mathrm{SF}_{6}$ gas environment. After laser ablation, a large number of micron papillary structures were distributed on the surface of the silicon. At the same time, abundant nanocomposite structures were attached to a single micron-scale synapse. Because the aforementioned preparation method is harsh on the environment, Chen et al. [84] started to construct superhydrophobic surfaces by using femtosecond lasers in air. In

(a)

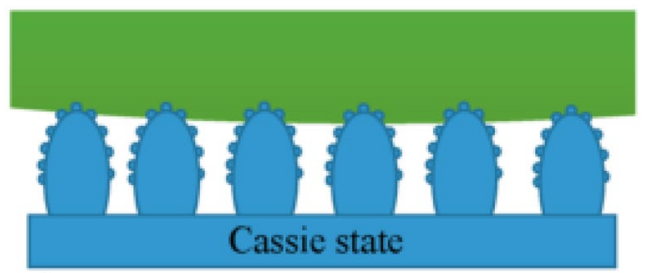

(b)

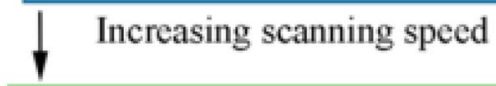

(c)

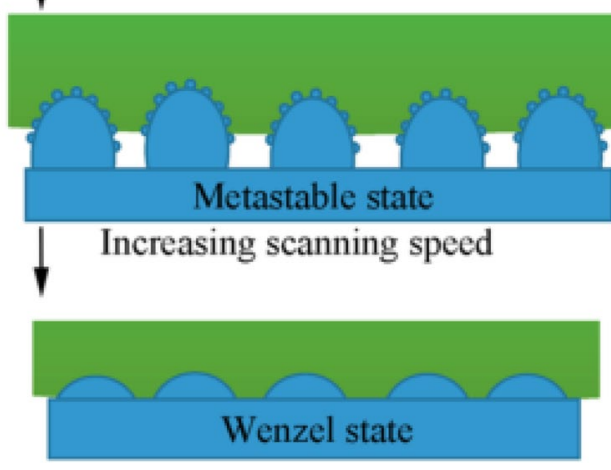

Fig. 29 Wettability of liquids on surfaces with different roughness values. a Contact of liquid in the Cassie model with a solid surface; b transition between the Cassie state and Wenzel state; c contact of a Wenzel model liquid with a solid surface [81] the process of progressive scanning, a periodic micro/nano composite structure was induced on the Si surface to form a micro-mountain structure similar to the microstructure on the lotus leaf surface. The micro-mountain had a diameter of approximately $6 \mu \mathrm{m}$ and a height of approximately $2.9 \mu \mathrm{m}$. The CA and sliding angle (SA)of the water droplets were $158^{\circ}$ and $4^{\circ}$, respectively. Water droplets could even bounce on the surface, and the surface had excellent superhydrophobic properties.

The free surface energy of intrinsically hydrophilic materials is high. It is necessary to construct a rough structure on the surface first and then use a low surface energy material to make surface modifications to impart superhydrophobic properties. Intrinsically hydrophobic materials can avoid this problem. Yoon et al. [85] used a femtosecond laser to etch PDMS to create a messy raised micro/nano composite structure. Coral-like structures of several micrometers were distributed on its surface and abundant nanoparticles covered the structure. The CA of $165^{\circ}$ and the SA as low as $2.1^{\circ}$ were achieved without further processing. In fact, the superhydrophobicity achieved on an intrinsically hydrophobic material is more stable and the wear resistance is stronger. After a certain amount of friction, the rough structure obtained after treatment is destroyed, but because of its hydrophobic nature, the newly exposed structure plays a certain hydrophobic role and the hydrophobic performance of the structure does not decrease significantly.

Glass is commonly used to fabricate optical components. Compared with other methods, the femtosecond laser can easily and accurately control the surface micromorphology of glass [86]. Ahsan et al. [87] obtained a superhydrophobic structure by subjecting the surface of soda-lime glass to femtosecond laser treatment. The CA of water droplets on the prepared surface reached $154^{\circ}$, and the transmittance of visible light was $77 \%$. Zhong et al. [88] used ultra-fast laser direct-writing-dispersed micropit array to fabricate the surface of silica glass. Adequate untreated areas ensure high transparency, and microdent arrays provide superhydrophobic roughness to the entire surface. Processing method schematic diagram and actual surface topography are shown in Figs. 30 and 31. The CA of the glass surface thus obtained can reach $161.6^{\circ}$, and the SA is approximately $1^{\circ}$. The transparency of visible light reaches $92 \%$, CA can be maintained substantially unchanged at a high temperature of $500{ }^{\circ} \mathrm{C}$, and impact resistance is strong.

Femtosecond laser fabrication can also impart superhydrophobicity to graphene materials. Shi et al. [89] used femtosecond laser single pulse ablation to prepare a threedimensional rose-like petal pattern on the surface of graphene, and each petal consisted of multiple tilted graphene nanosheets (Fig. 32). The single pulse ablation method helped achieve a patterned structure with a large area. 
Fig. 30 Sketch map of three different ways of femtosecond laser treatment of glass surface: a identical pulse energy, b gradient-decreasing pulse energy, and $\mathbf{c}$ gradient-increasing pulse energy and morphology of glass surface after treatment [88]
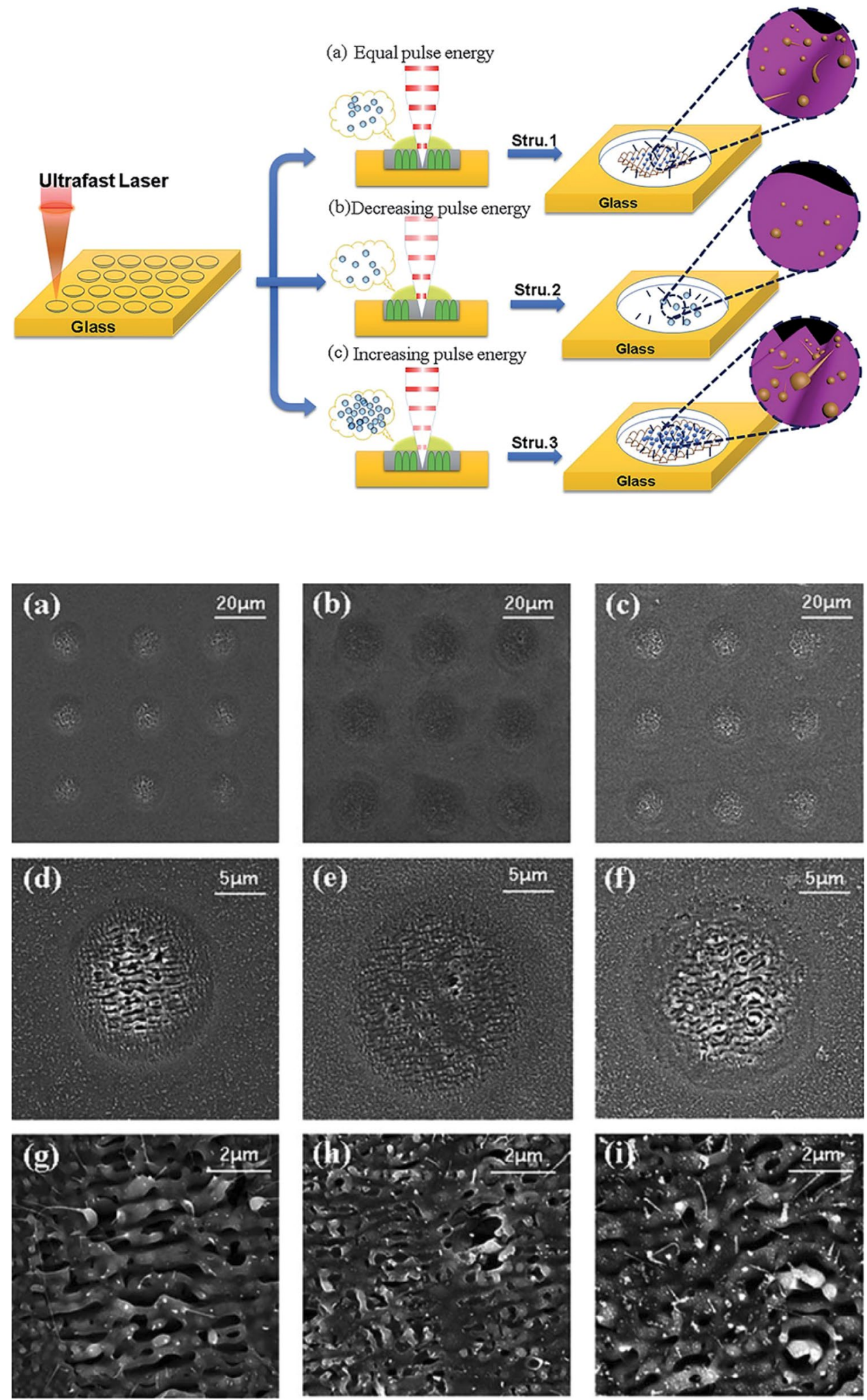

In addition, imparting superhydrophobicity to metals can effectively help avoid metal pollution, corrosion, and rust. The pattern fabricated using a femtosecond laser on a metallic surface becomes a part of the metal material that cannot be rubbed off or wear easily, and the hydrophobicity achieved this way is superior. Zhong et al. [81] have 


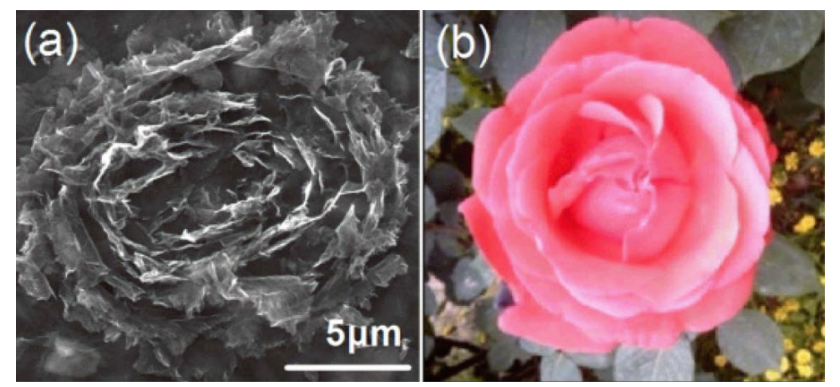

Fig. 32 a Rose pattern formed on surface of graphene after femtosecond laser fabricating; $\mathbf{b}$ roses in nature [89]

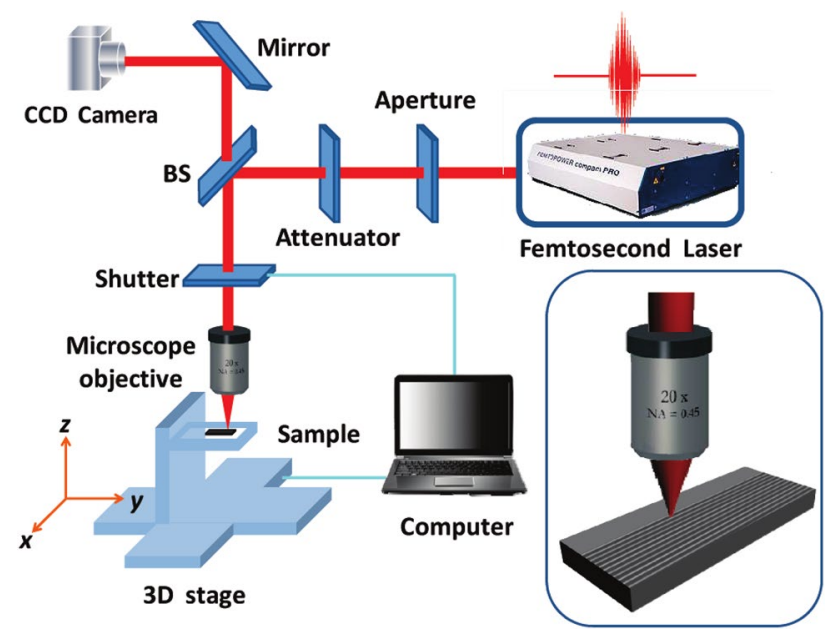

Fig. 33 Schematic of experimental device for femtosecond laser scanning [81]

studied the effect of different micro/nanostructures about the adhesion of droplets on copper surface. By adjusting the laser scanning speed under certain parameters, the depth of periodic surface microstructures and the richness of nanostructures can be controlled accurately to realize continuous adjustment of surface adhesion force. The schematic diagram of the processing device and the different surface topography structures are shown in Figs. 33 and 34. Surfaces formed at different scanning speeds have different adhesion forces to water, and SA can vary from $1^{\circ}$ to $90^{\circ}$. Shin et al. [90] realized superhydrophobicity on three-dimensional copper surface. There are many wave packets on the surface of copper (Fig. 35). This technology is expected to be used in microfluidic devices.

After laser ablation, the superhydrophobic properties mentioned above can only show a single wetting state. To achieve reversible hydrophilic and hydrophobic transformations, Chen et al. [91] used a femtosecond laser to fabricate the surface of metallic $\mathrm{Zn}$ and developed a superhydrophobic intelligent structure with wet-state changes. The reversible change from superhydrophobic to superhydrophilic was achieved by alternating treatments of ultraviolet radiation and dark storage. A micro/nano layered composite structure was formed on the surface by femtosecond laser ablated, and the metal was oxidized as well. Meanwhile, the $\mathrm{ZnO}$ formed by ablation and oxidation was a photosensitive semiconductor oxide material and underwent a characteristic reaction through ultraviolet light and dark treatment. The processing methods, surface micro-morphology and results of different materials are summarized in Table 4.

\subsubsection{Superoleophobic Surface and Oil-Water Separation}

Because the surface tension of oil is much lower than that of water, it is more difficult to construct superoleophobic structures than superhydrophobic structures [92, 93]. To obtain superoleophobic surfaces in air, inner-corner bending structures and strict chemical modification are needed to reduce surface energy, except in the case of rough micro/ nano hierarchical structures [94, 95].

The surface of fish scales has underwater superoleophobicity [96]. The surface microstructure of fish scales is shown in Fig. 36. According to the extended Cassie theory [93, 97], underwater superoleophobicity is achieved because liquid droplets stay in the layered micro/nano roughness of the material surface and oil droplets are attached to the water molecules to form an oleophobic layer.

Chu et al. [98] used femtosecond laser direct writing techniques to construct periodic micro/nanostructures on sapphire wafers. The surface has good underwater superoleophobicity, and the oil contact angle (OCA) in water can reach $153^{\circ}$ with good stability. By means of liquid-assisted femtosecond laser ablation, Li et al. [99] imparted superoleophobicity to the surface of silicon. The OCA on this surface reached $169.21^{\circ}$. Wu et al. [100] employed a femtosecond laser to scan nickel sheets placed in sucrose solution. The results showed that many microsphere arrays were formed on the nickel plate, and many nano-fluffs were observed on the surface of each microsphere as shown in Fig. 37. And the height and size of the cone could be adjusted accurately and the wettability of water and oil could be controlled.

With respect to oil-water separation, conventional oil-water separation methods are limited by poor reliability, complicated preparation processes, and low reusability. The use of femtosecond laser fabrication can help to effectively overcome these limitations [101-103], and it was employed to fabricate an ultrathin aluminum foil to form a novel film consisting of nanostructured microporous arrays by a femtosecond laser, which can realize underwater superoleophobicity $\left(\mathrm{OCA}>150^{\circ}\right)$ and an oil-water separation function. In addition, microholes with a strong filtering effect for particles of different sizes were fabricated on a titanium foil by femtosecond laser microhole drilling, as shown in Figs. 38 and $39[104,105]$. Duan et al. [106] fabricated stainless-steel 

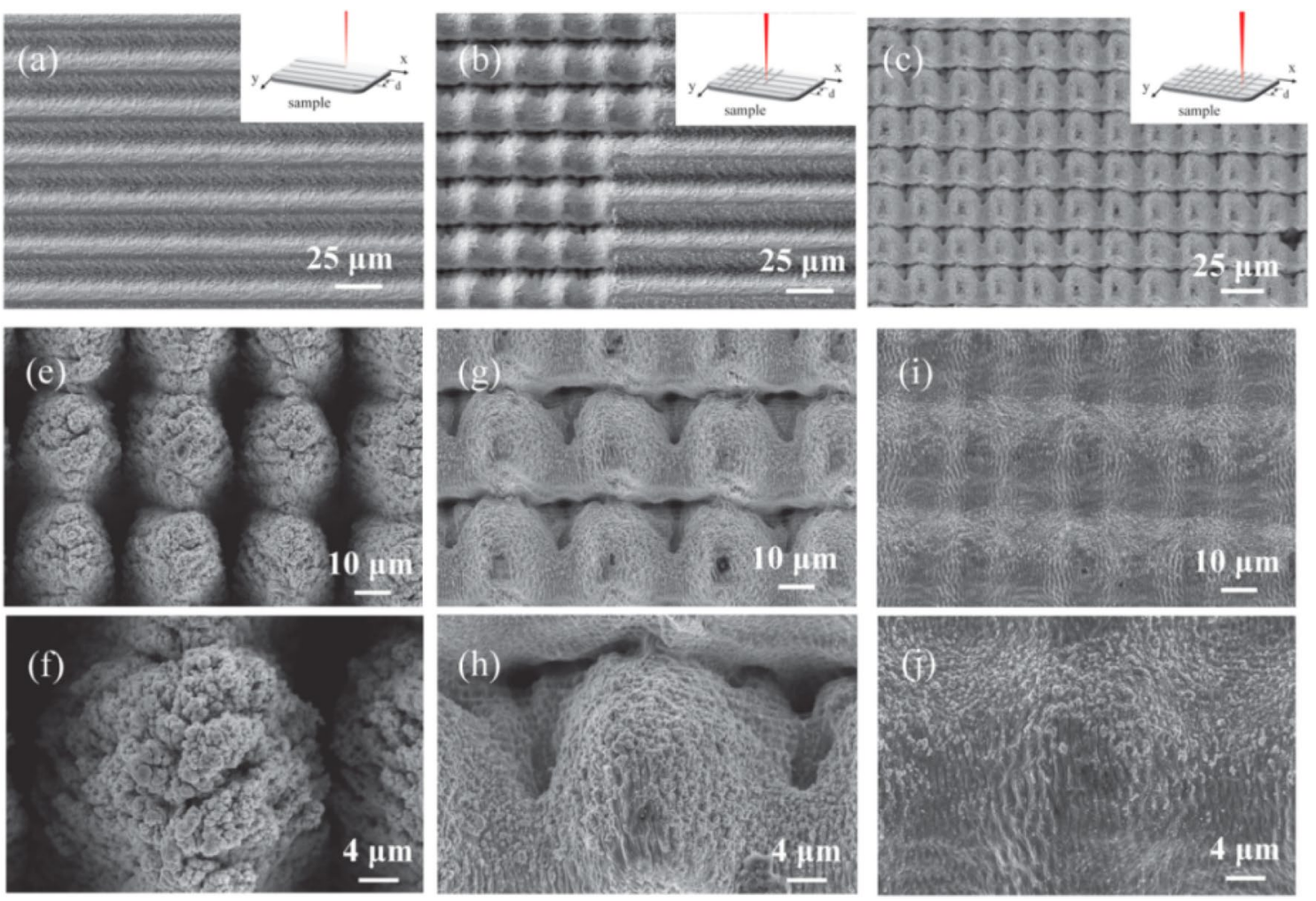

Fig. 34 a-c Changes in surface morphology of copper at different times during femtosecond laser scanning; $\mathbf{d}-\mathbf{i}$ SEM images of copper surface structured using femtosecond laser with different scanning speeds: d, e $10 \mathrm{~mm} / \mathrm{s}, \mathbf{f}, \mathbf{g} 50 \mathrm{~mm} / \mathrm{s}$, and h, i $100 \mathrm{~mm} / \mathrm{s}$ [81]

Fig. 35 a SEM image of periodic micro-bumps structure formed on copper surface; $\mathbf{b} 3 \mathrm{D}$ optical profiler image of a textured copper micro-bumps [90]

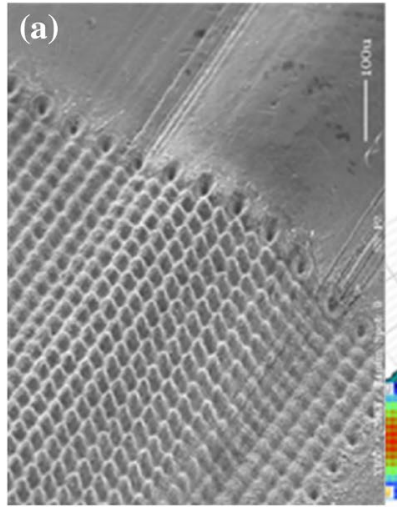

(b)

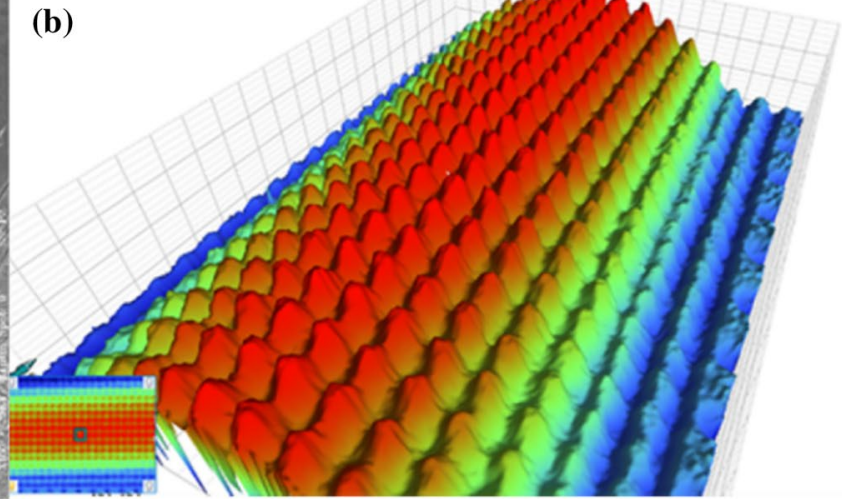

grids with nanostructures (Fig. 40). The oil-water separation efficiency of these structures was greater than $99 \%$, and structures were corrosion resistant. Yong et al. [107] prepared a superhydrophobic/superlipophilic PTFE mesh by using a femtosecond laser. The surface can even maintain a high degree of oil-water separation efficiency in strongly acidic $(\mathrm{pH} \sim 1)$ or strongly alkaline $(\mathrm{pH} \sim 13)$ environments.

However, many problems remain unsolved in the research on special wettability by femtosecond laser fabrication: (1) Low efficiency, at present, femtosecond laser fabrication is relatively time consuming, and the processing efficiency cannot satisfy the requirements of industrial production; (2) Low stability, surfaces that can withstand friction, high temperature, acid/alkali corrosion, and work for a long time is still the main research direction. (3) The existing laser fabrication technology mainly focuses on the two-dimensional surface structure. Three-dimensional complex structures such as pipes and cavities are of great significance to practical applications. 
Table 4 Summary of superhydrophobic properties on different material surfaces

\begin{tabular}{|c|c|c|c|c|c|}
\hline Material & Surface topography & Processing method & $\mathrm{CA}$ & SA & Remarks \\
\hline Silicon [83] & Micron papillary & $\begin{array}{l}\text { Femtosecond laser direct } \\
\text { writing }\end{array}$ & $154^{\circ}$ & $5^{\circ}$ & - \\
\hline Silicon [84] & Micro-mountain & Progressive scanning & $158^{\circ}$ & $4^{\circ}$ & - \\
\hline PDMS [85] & Coral-like structures & $\begin{array}{l}\text { Femtosecond laser direct } \\
\text { writing }\end{array}$ & $165^{\circ}$ & $2.1^{\circ}$ & $\begin{array}{l}\text { The transmittance of visible } \\
\text { light is } 77 \%\end{array}$ \\
\hline Soda-lime glass [87] & $\begin{array}{l}\text { Periodic microgratings and } \\
\text { micro-pillars }\end{array}$ & $\begin{array}{l}\text { Single Beam Direct Writing } \\
\text { (SBDLW) }\end{array}$ & $152^{\circ}-155^{\circ}$ & - & $\begin{array}{l}\text { The transparency of visible } \\
\text { light is } 92 \% \text {; high temperature } \\
\text { resistance, shock resistance }\end{array}$ \\
\hline Silica glass [88] & $\begin{array}{l}\text { Micro-nano rods, ripples, } \\
\text { particles }\end{array}$ & $\begin{array}{l}\text { Ultra-fast laser direct-writing- } \\
\text { dispersed micropit array }\end{array}$ & $161.6^{\circ}$ & $1^{\circ}$ & High-efficiency \\
\hline Graphene [89] & Rose-like petal pattern & Single pulse ablation & $150^{\circ}$ & - & \\
\hline Copper [81] & Micropits, nanofolds & $\begin{array}{l}\text { Femtosecond laser direct } \\
\text { writing }\end{array}$ & $140^{\circ}-160^{\circ}$ & $1^{\circ}-90^{\circ}$ & \\
\hline Copper [90] & $\begin{array}{l}\text { Micro bumps and micro } \\
\text { grooves }\end{array}$ & $\begin{array}{l}\text { Femtosecond laser direct } \\
\text { writing }\end{array}$ & $147^{\circ}$ & $48^{\circ}$ & $\begin{array}{l}\text { Achieving superhydrophobicity } \\
\text { on three-dimensional curved } \\
\text { surface }\end{array}$ \\
\hline Zinc [91] & Synapse & $\begin{array}{l}\text { Femtosecond laser direct } \\
\text { writing }\end{array}$ & $\begin{array}{l}14.5^{\circ}-80.6^{\circ} \\
\text { and } 116.6^{\circ}- \\
159.5^{\circ}\end{array}$ & - & $\begin{array}{l}\text { Transformation from superhy- } \\
\text { drophobicity to hydrophilicity }\end{array}$ \\
\hline
\end{tabular}
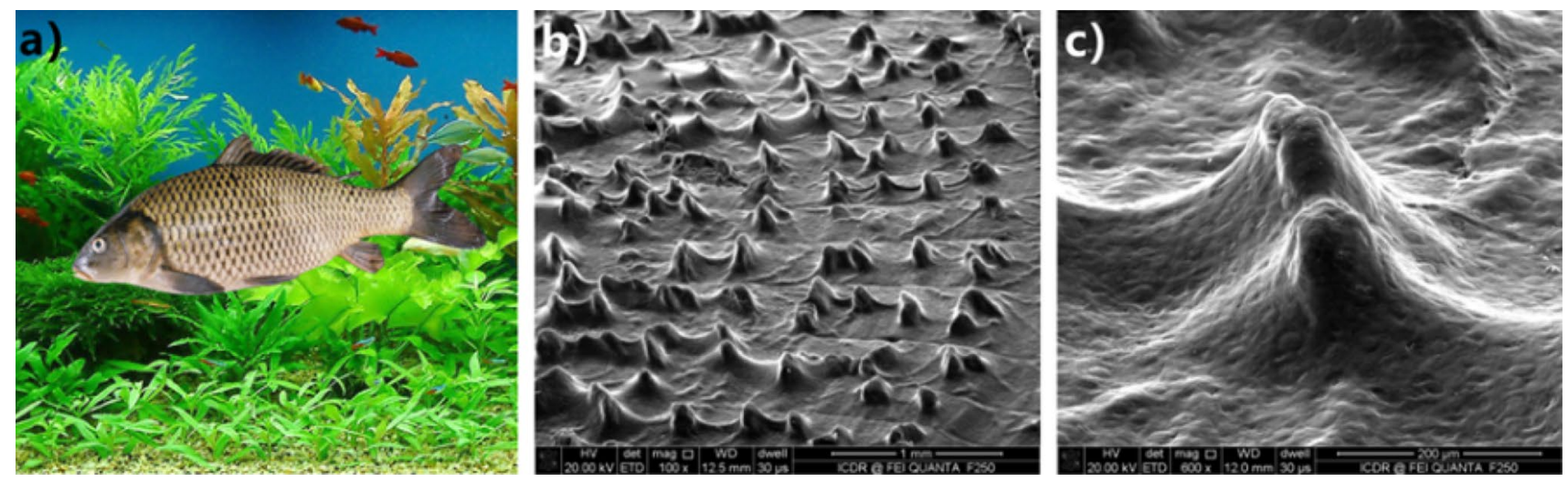

Fig. 36 SEM images of fish scale surface [96]

Fig. 37 a Sketch map of microcone arrays formed on a nickel sheet after treatment with a laser. b Height and radius of each microcone. $\mathbf{c}$ CAs of oil droplets in air and underwater after treatment. d SEM images of morphology of surface structures prepared in sucrose solution [100]

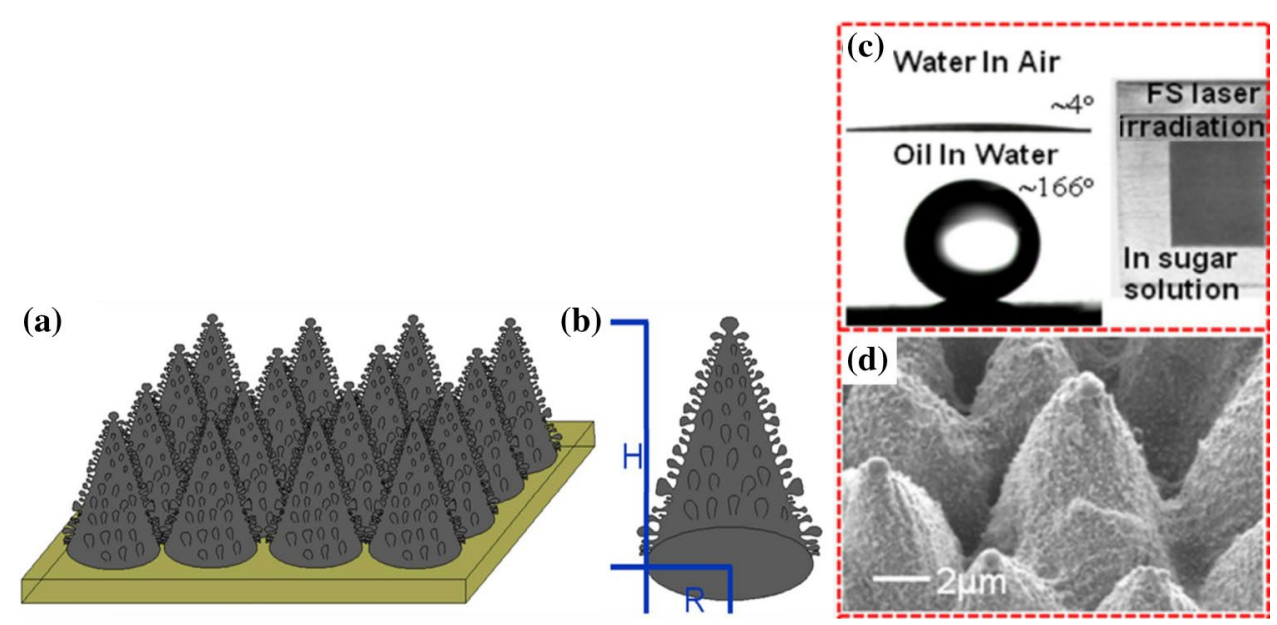




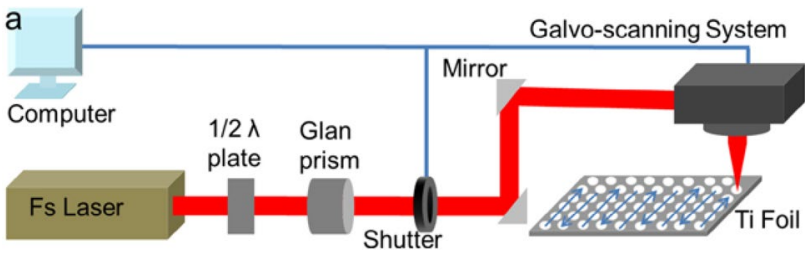

Fig. 38 Schematic of drilling microholes on titanium foil [104]

\subsection{Biomedical Applications}

Femtosecond laser fabrication is especially suitable for biological applications because it can be applied to any type of biological tissue or cells with high precision, a small heat-affected zone, and three-dimensional controllability [108-113]. Femtosecond lasers can be used to label or transfect cells. Most cells are transparent in the near-infrared range. When a femtosecond laser is focused on a transparent material, the intensity at the point of focus is high enough to cause nonlinear absorption [113-115]. Researchers have been using femtosecond lasers instead of electron waves to perforate cell membranes. When a laser beam is focused on a membrane for a few milliseconds, transient holes are created through which proteins, RNA, or DNA can enter the cell for labeling or transfection [116, 117]. Femtosecond lasers can also be used for microscopic imaging, which is characterized by high-fluorescence resolution in intact tissues [118-121]. Chu et al. [122] irradiated Hoechst-labeled hepatocytes with a femtosecond laser and obtained three-photon-fluorescence and third harmonic generation to reveal the location of the cytoplasmic membrane and other membrane-based cellular organs such as mitochondria.

Femtosecond laser was used to operate on organelles in living cells. Traditional cutting tools, such as microneedles, are invasive and often destroy the connection between structures. Femtosecond laser surgery can provide sub-micron resolution, minimally alter the surrounding environment of cells and can be applied to living biological samples [123, 124]. This nanosurgical technique provides an effective noncontact method for manipulating and removing fragments of organelles or chromosomes from within a single living cell.

Femtosecond laser fabrication technology for biological micro-devices has attracted wide attention. Danilevicius et al. [125] used the multiphoton polymerization method combined with flexible laser direct writing to create a $9 \times 9$ $\times 2 \mathrm{~mm}^{3}$ microdisc type 3D bioscaffold and a hollow tubular vascular scaffold of approximately $10 \mathrm{~mm}^{3}$ on SZ2080 polymer and Ormoclear polymer. Moreover, the size of the micropore on the scaffold can be arbitrarily adjusted between 10 and $100 \mu \mathrm{m}$, so that the shape of the scaffold can be accurately controlled to optimize its mechanical performance, as shown in Figs. 41 and 42. At the same time, the microstructure prepared by this method has good compatibility with biological tissues. Femtosecond laser multi-photon fabrication technology makes it possible to fabricate arbitrary structure, pore size, and pore density quickly, which provides a convenient and fast method for
Fig. 39 SEM images of surface microstructure of titanium foil after femtosecond laser fabrication [105]

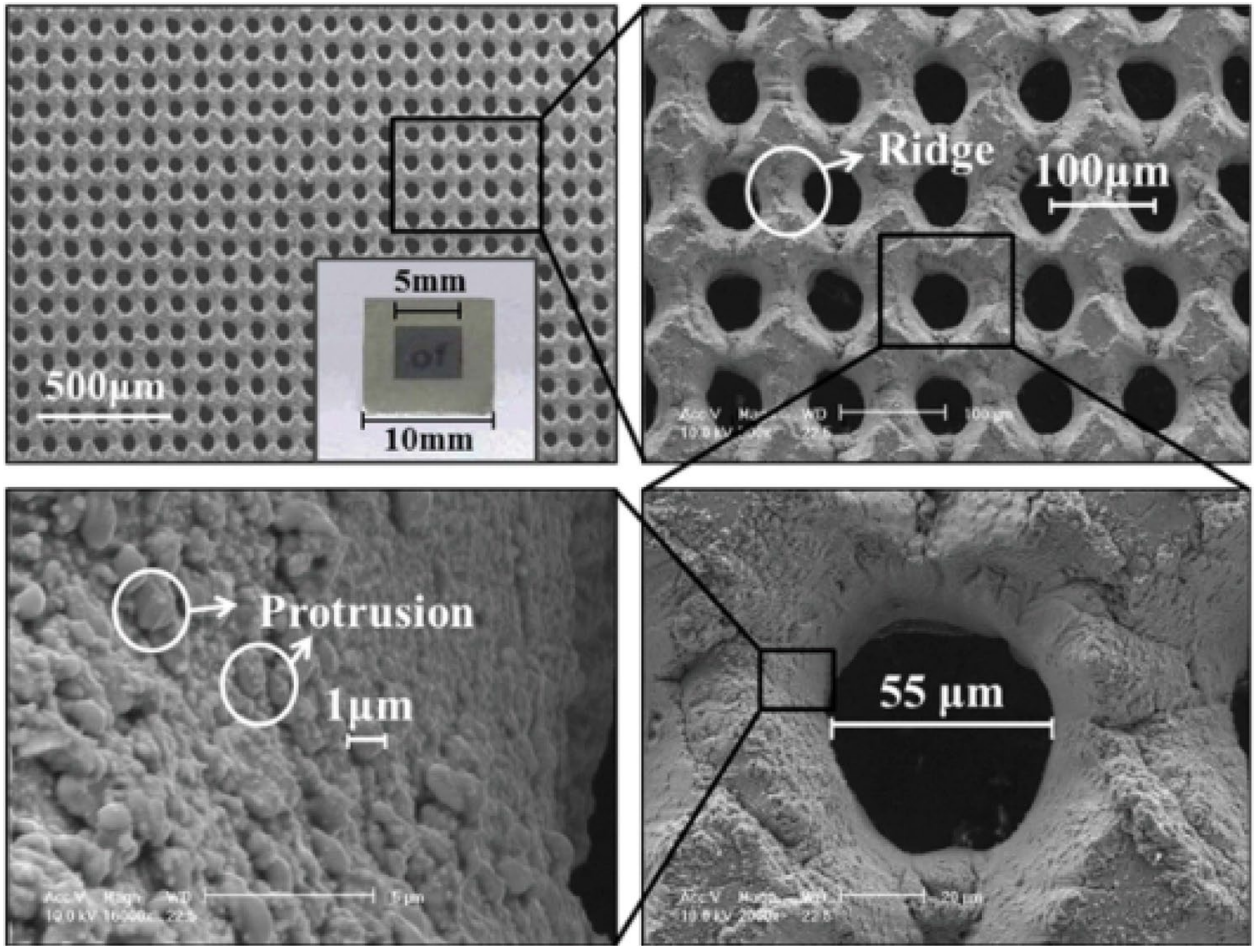


Fig. 40 a Untreated stainless steel grid and stainless-steel grid after laser fabrication; b SEM images of pristine stainless-steel mesh; c-e SEM images after laser fabrication at different magnifications [106]
Fig. 41 Multiphoton polymerization fabrication steps: (I) photopolymerization at the laser focus; (II) processing samples by laser direct writing; (III) organic developer washes unexposed material; (IV) processed 3D scaffold [125]
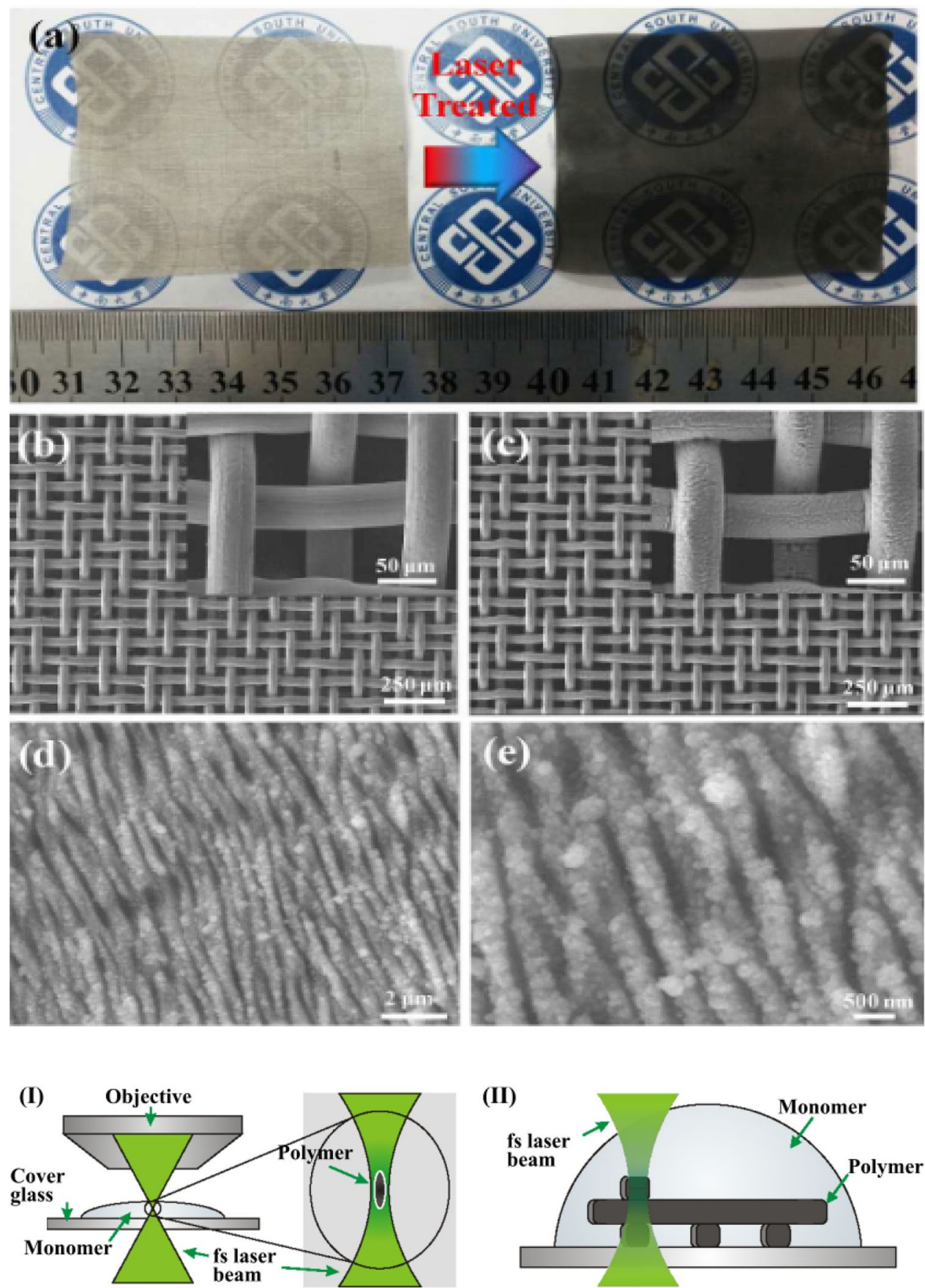

(II)

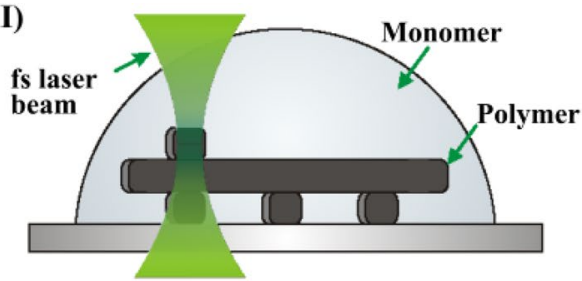

(III)

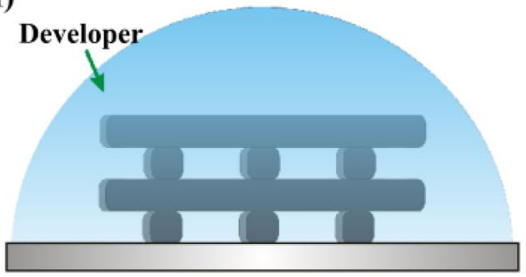

(IV)

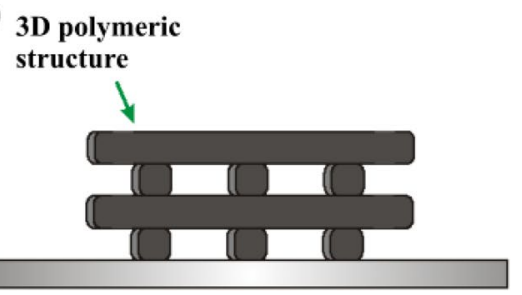



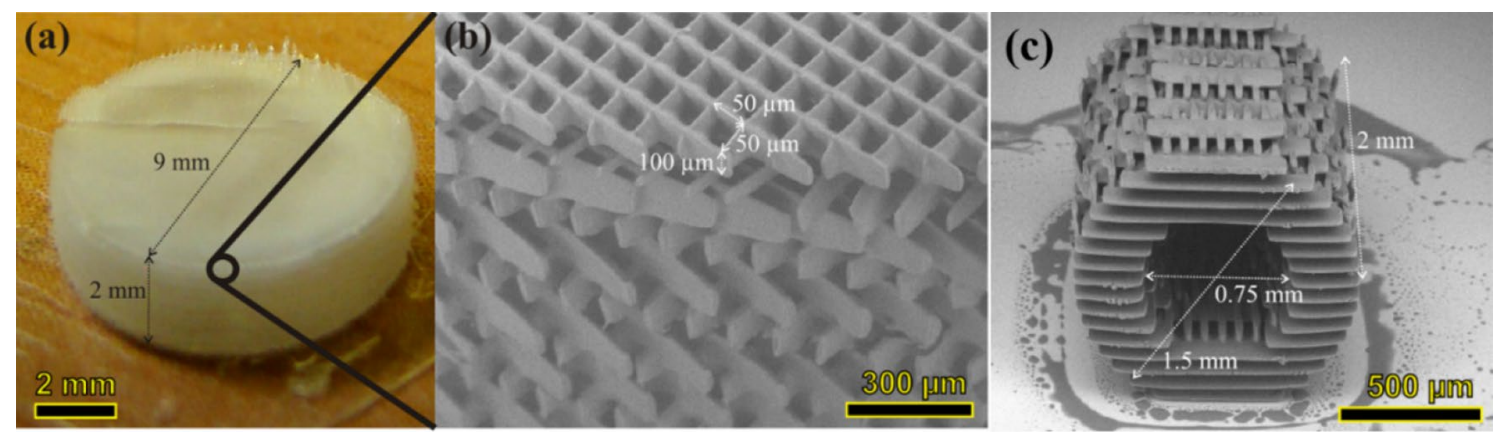

Fig. 42 a $9 \times 9 \times 2 \mathrm{~mm}^{3}$ microdisc type $3 \mathrm{D}$ bioscaffold on SZ2080 polymer; b partially enlarged small pore of $50 \times 50 \times 100 \mu \mathrm{m}^{3}$; $\mathbf{c}$ SEM image of 3D blood vessel scaffold fabricated on Ormoclear polymer [125]

optimizing and quantifying the three-dimensional structure and realizes the rapid fabrication of nano-precision and it is of great significance for the fabrication of highperformance cell scaffolds.

In addition, for processing proteinaceous materials, femtosecond lasers offer a distinct advantage. Unlike nanoimprinting and ultraviolet lithography [126, 127], femtosecond laser direct writing offers an excellent threedimensional fabrication ability and can be used to produce devices with nanometer precision. More importantly, biomaterials prepared using femtosecond laser direct writing can retain the biological activity of biomaterials [128, 129]. Kaehret al. [130] created a three-dimensional (3D) cell culture microcavity based on bovine serum albumin (BSA) through mask-directed multiphoton lithography. The microcavity can capture and culture individual cells and detect cell properties in a controlled environment (Fig. 43). Spivey et al. [131] obtained complex 3D suspended protein micromechanical devices in environments with extremely high protein concentrations. This waterphase direct writing technology can be used to fabricate a suspended 3D device.

\subsection{Data Storage Materials and Devices}

The information age has led to the rapid development of data storage technology. Holographic technology, near-field optical storage technology, and laser-induced storage technology have been applied to research on data storage [132-135].
The materials used commonly for optical storage are phase transition materials and nanometer grating structures [136, 137]. Ultrafast lasers can accurately deposit energy in transparent materials and produce the nonlinear characteristics of femtosecond laser interaction with materials, resulting in phase transition or varieties of refractive indexes, which makes it the most suitable method for fabricating memory devices $[138,139]$.

To overcome the limitations of storage capacity and improve data storage density, optical storage technology has been improved from three dimensions to five dimensions. The first digital document encrypted optically into five dimensions was retrieved successfully by nondestructive quantitative birefringence measurements [137]. A process for recording multiplexed digital data was implemented by femtosecond laser nanostructuring of fused quartz (Fig. 44). Multilevel encoding of intensity and polarization states of light with self-assembled nanostructures has increased the data recording rate by two orders of magnitude.

Photobleaching materials, photochromic materials, and micro-explosive materials have been studied from the viewpoint of data storage. In 2009, plasmonic gold nanorods were realized for five-dimensional optical recording by exploiting the unique properties of longitudinal surface plasmon resonance of gold nanorods [140]. Then, Royon et al. [141] created silver clusters through a femtosecond laser shot inside a femtophotoluminescent glass, which can store information precisely. Klyukin et al. [142] observed the presence of fluorescent clusters in chloride photothermorefractive glass under femtosecond laser irradiation. Birefringent
Fig. 43 a, b SEM images of BSA microcavity, c SEM image of cross-linked BSA plug at microcavity entrance [130]
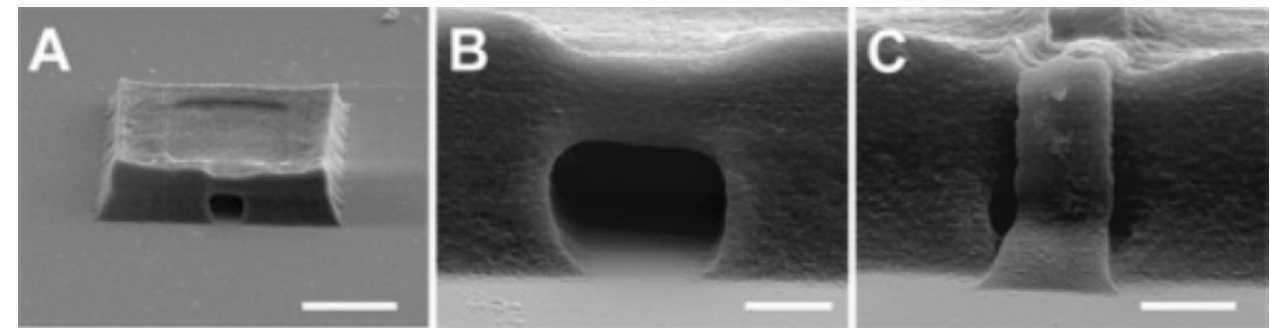
Fig. 44 a Microstructure induced by femtosecond laser; $\mathbf{b}, \mathbf{c}$ are nanograting calculation results with different intensities [137]

(a)

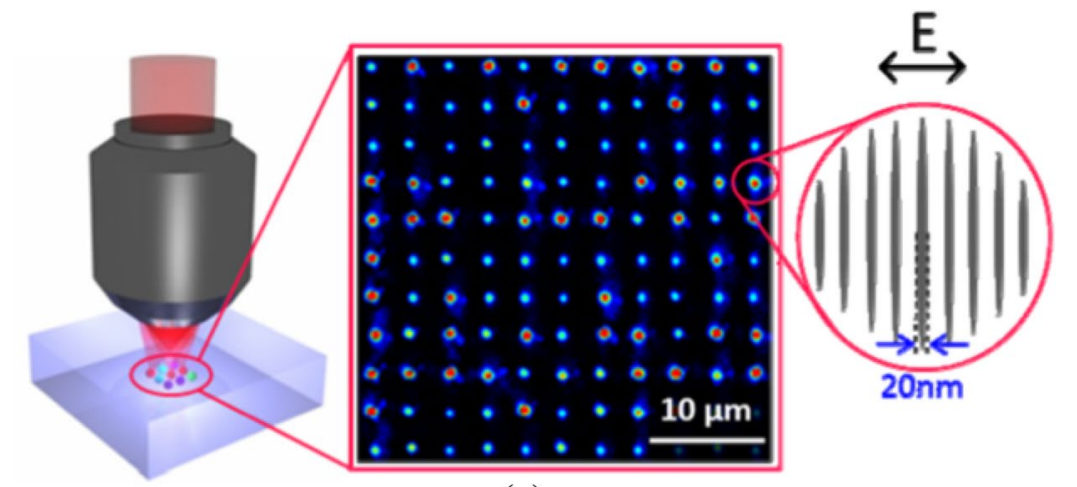

(b)

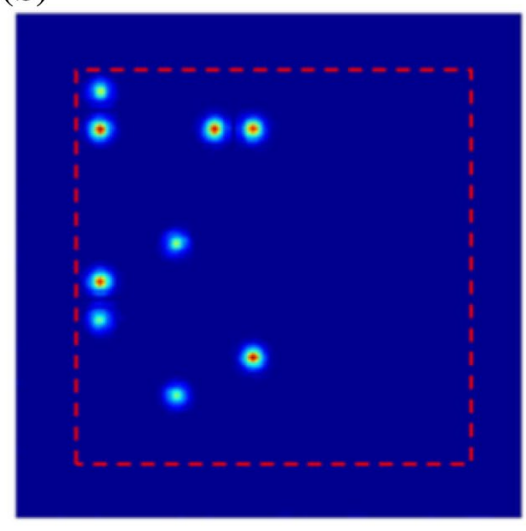

(c)

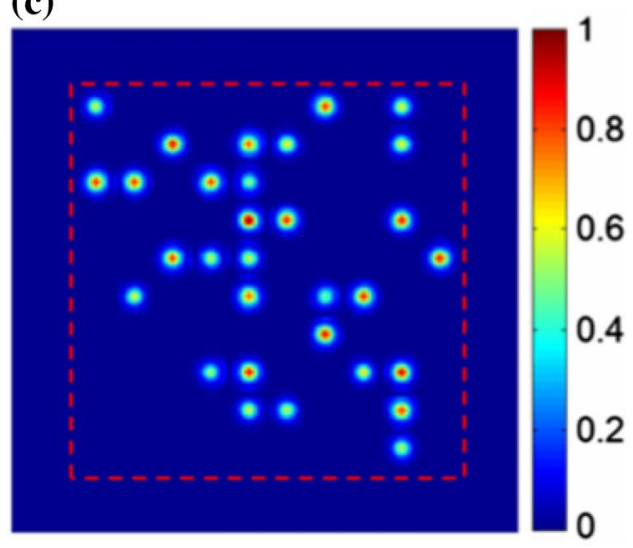

structures were obtained by means of multiple femtosecond laser pulses in nanoporous glass, where the minimum distance between dots was as small as $1.4 \mu \mathrm{m}$ [143]. This structure improved information density, thus facilitating the recording of three bits of information inside nanoporous glass. Cerkauskaite et al. [144] obtained 120-nm periodic deep subwavelength ripples on indium-tin-oxide thin film by means of femtosecond laser direct writing. The form birefringence was much higher than that of uniaxial crystals and fused-quartz nanostructures. Moreover, the data storage capacity of these nanostructures was four times higher than that of conventional materials.

With the further research on the interaction of femtosecond lasers and materials, new storage materials and techniques have been developed continuously, providing many possibilities for information storage. Currently, the main technical problems in the data storage field are improving storage capacity, increasing information reading speed, ensuring material stability, and processing efficiency.

\subsection{Carbon-Based Materials Processing}

Since the successful exfoliation of graphene by Heim et al. in 2004, graphene has been utilized extensively because of its excellent physicochemical properties such as high light transmittance, excellent mechanical properties, extremely high electron mobility, and high thermal conductivity [145].
Numerous studies have shown that the surface patterning of graphene and related materials has major theoretical and practical applications in supercapacitors, biosensors, solar devices, and other fields [146-151]. However, the traditional mechanical peeling of single-layer graphene and graphene patterning have low processing efficiency and low precision and are unsuitable for large-scale and fine preparation.

The use of femtosecond laser technology for stripping graphene is effective for solving these problems. Ablating CVD monolayer graphene through single-shot femtosecond pulses with different laser beam diameters and laser fluences provides a reference for studying graphene single-shot ablation probabilities [152]. Yoo et al. [153] developed a novel laser-induced pattern transfer method that reduces the complexity of graphene fabrication. The relevant flow diagram is depicted in Fig. 45. Graphene/PMMA patterns were processed on several types of substrates, and the method showed potential with respect to fabricating other functional devices.

Graphene nanostructures measuring only $80 \mathrm{~nm}$ in size, which is far beyond the diffraction limit, were obtained using a space-shaped femtosecond laser [154]. The entire process was very accurate and convenient because the shaping and imaging were carried out under a confocal microscope. Dong et al. [155] used femtosecond laser deposition to fabricate large-area few-layer graphene at a certain pressure and temperature. The average thickness of the resulting graphene film was less than $3 \mathrm{~nm}$, and the average area was more than 
Fig. 45 Flow diagram of the laser-induced pattern transfer method [153]

\section{(a) Attachment step}

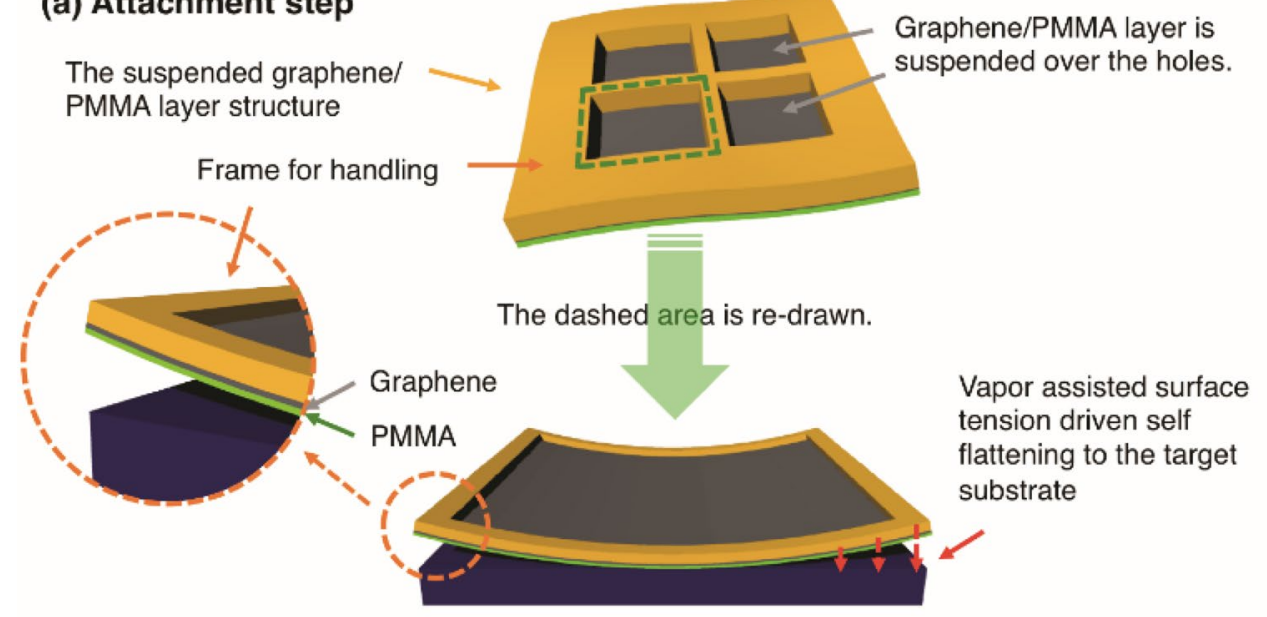

(b) Direct patterning and simultaneous transferring step

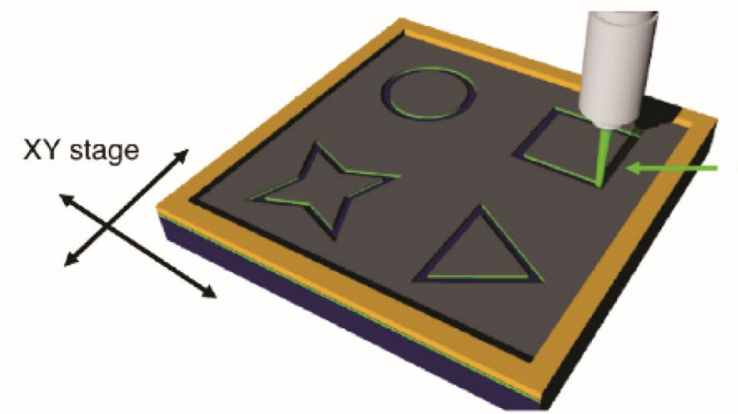

Ultrafast laser ablation

(c) Detachment step
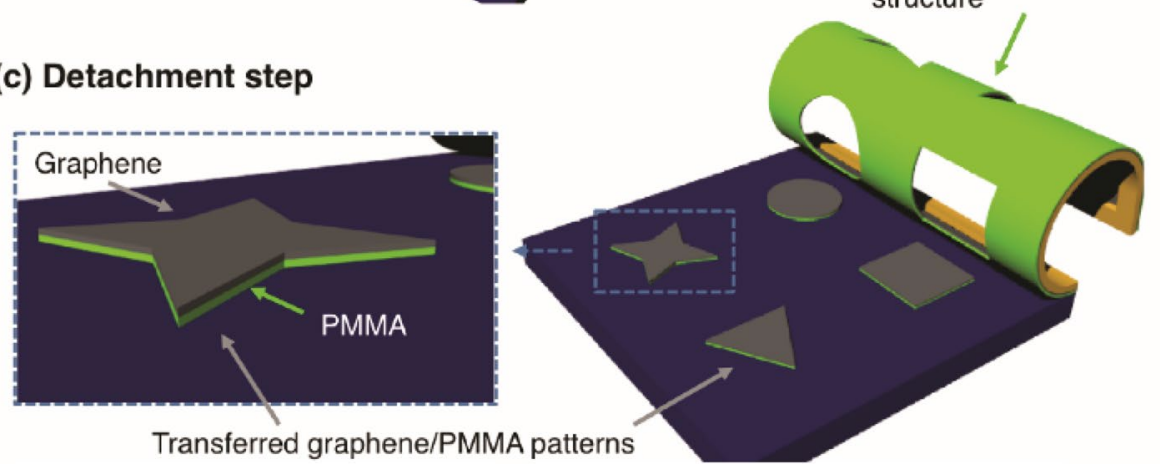

$1 \mathrm{~cm}^{2}$. A spontaneous periodic graphene microstructure was processed under femtosecond laser irradiation [156]. The optical properties of several different irradiation regions were discussed, and it was proved that they were related to different modification mechanisms.

In recent years, femtosecond laser pulse irradiation has become suitable for processing of carbon-based materials, such as graphene oxide (GO) reduction [157-160]. Complex patterns on GO films can be created through direct femtosecond laser reduction [161]. Femtosecond laser direct writing can reduce GO to graphene efficiently and flexibly [162, 163]. Different laser parameters, such as laser fluence and irradiation time, were applied to obtain the optimum reduced GO with fewer defect sites and lower electrical resistivity. The interplanar spacing between carbon layers decreased to $3.51 \AA$, which is close to that of pristine graphite. Apart from direct writing and ablation, fs laser can be used to detect the optical behaviors of graphene or GO nanostructures. Recently, femtosecond laser pump-probe measurement has been applied to detect the nonlinear optical response of GO and gold nano composites prepared by femtosecond laser ablation [164]. The enhance of nonlinear optical response was demonstrated by femtosecond laser pump-probe 
Fig. 46 a Decay process measured by laser time-resolved pump-probe measurement and $\mathbf{b}$ band gap of the nanostructure [164]

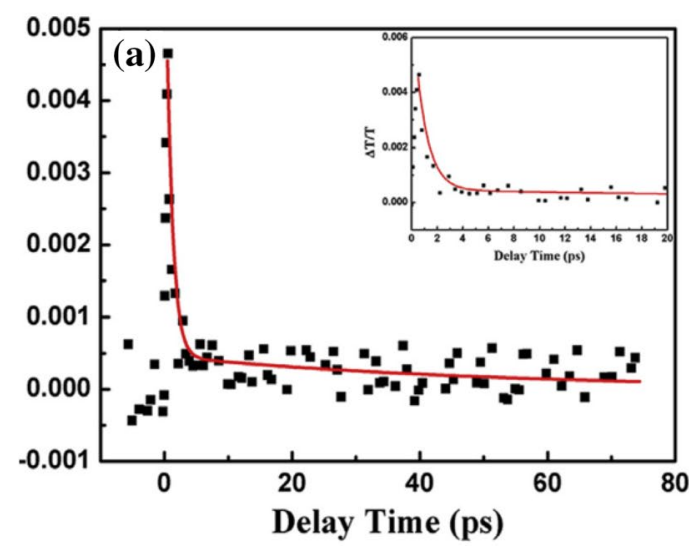

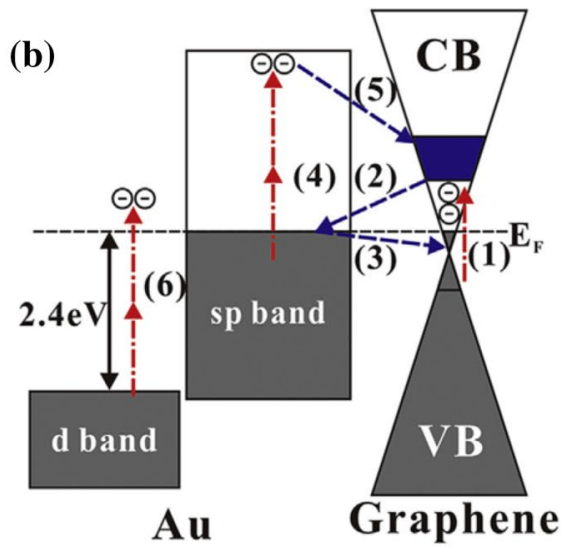

measurement, and the shifted negative absorption peak with laser power increasing was observed and explained to be related with sp band transition. Figure 46 shows the decay process and the sketch map of nanostructure energy band.

The combination of femtosecond laser irradiation and graphene-based devices stimulated the development of more accurate and effective preparation methods, thus providing us with new research ideas.

\subsection{High-Aspect-Ratio Microholes}

In precision manufacturing, microholes are common and key structural elements. Especially, high-quality and highaspect-ratio microholes play vital roles in major national requirements such as aerospace gas turbines $[165,166]$, the ignition target in inertial confinement fusion ignition engineering [167, 168], high-sensitivity sensors [169], photonic crystal fibers [170], and engine injectors [171]. Basically, microhole fabrication requires high-quality (no recast layer, no crack, no heat-affected zone, uniform density) and various materials (alloys, polymers, glass, etc.). Especially, in a few special cases, microholes must have a high aspect ratio (10:1 to 100:1 or even higher) and be nonpolluting [53, 167]. Femtosecond laser has the advantages of minimizing heataffected zone, less recasting layer, non-contact in processing, and no need of liquid/acid-base assistance and it has become one of the most promising processing methods to fabricate high-aspect-ratio microholes [167, 172-175].

In terms of processed materials, it can be divided into metal materials and non-metal materials. For metal materials, due to the complex interaction environment and many influencing factors during femtosecond laser fabrication, the shape of micro-hole exit is irregular and the processing quality is poor, besides the processing results are not easy to observe. Therefore, it is more difficult to fabricate high-aspect-ratio microholes on metals by femtosecond laser [176-179].
Unlike metal materials, femtosecond lasers can achieve high-efficiency ablation of transparent materials because of their nonlinear ionization effect, and their nonthermal phase transition mechanism can lead to high-quality processing results $[53,180]$. Large aspect ratio is very important for microholes. Shah et al. [180] used the lens with long focal length to focus the femtosecond laser on transparent silicate glass and obtained the high-quality with diameter of about $100 \mu \mathrm{m}$ and the aspect ratio of 10:1 microporous structure in 2001. Nakashima et al. [181] obtained $470 \mathrm{~nm}$ diameter and the aspect ratio is 17:1 nanoholes in semiconductor gallium nitride $(\mathrm{GaN})$ by single pulse fabrication. However, the removal of materials by single pulse fabrication is limited, so it is more suitable for processing thin materials or blind holes with smaller depth. Eaton et al. [182] pointed out that the plasticity of materials can be improved by the preceding pulses and the subsequent pulses can process the material more effectively after a certain time delay. By using femtosecond laser pulse trains, high-aspect-ratio and high-quality microholes can be obtained on brittle materials. The Jiang group has done a lot of research on pulse trains fabrication, and proposed the femtosecond laser fabrication method based on dynamic electronic control. They used the dual temperature model to explain that the use of femtosecond laser pulse chains for microholes fabrication is more efficient than single pulse laser fabrication, and can greatly improve the aspect ratio of microholes to $50: 1$ by pulse trains [183-185].

Compared with Gaussian beam, Bessel beams are suitable for processing deep microholes because of its long focal length. The intensity distribution simulations of Bessel beam and Gaussian beam are shown in Fig. 47 [186]. In 2010, Bhuyan et al. [187] used a femtosecond laser to generate Bessel beams through the SLM and drilled high-aspectratio nanochannels on glass. A series of parallel nanochannel arrays with diameter ranging from 200 to $800 \mathrm{~nm}$ were obtained, and the aspect ratio exceeded 100 . However, the material of the SLM is easy to be damaged by high power 
Fig. 47 Intensity distribution simulations of Bessel beam and Gaussian beam [186]
Fig. 48 Experimental setup of microhole drilling using Bessel beams. ( $M$ plane mirror, $H W P$ half-wave plate, $P B S$ polarized beam splitter, $A$ aperture, $L$ plano-convex lens, $D M$ dichroic mirror, $M O$ micro objective, $S$ white-light sources) [188]
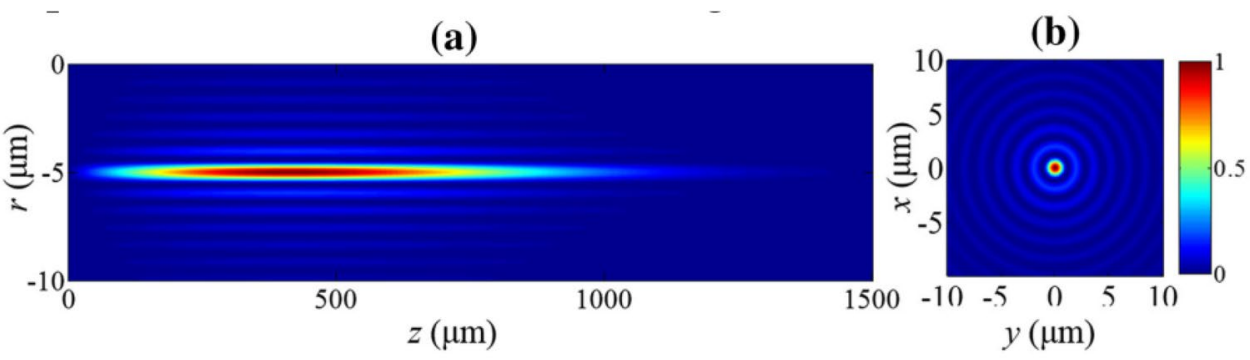

(c)

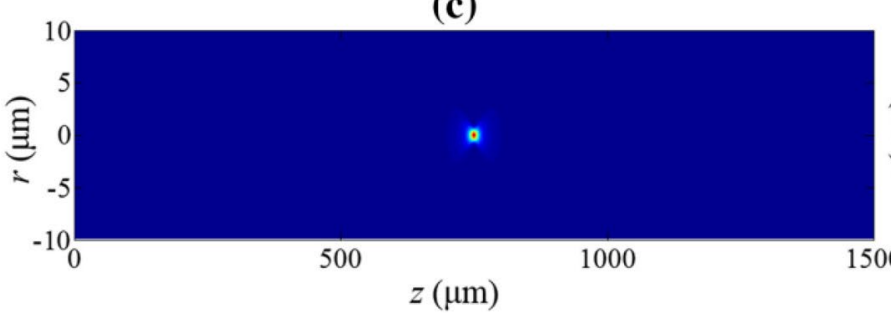

(d)
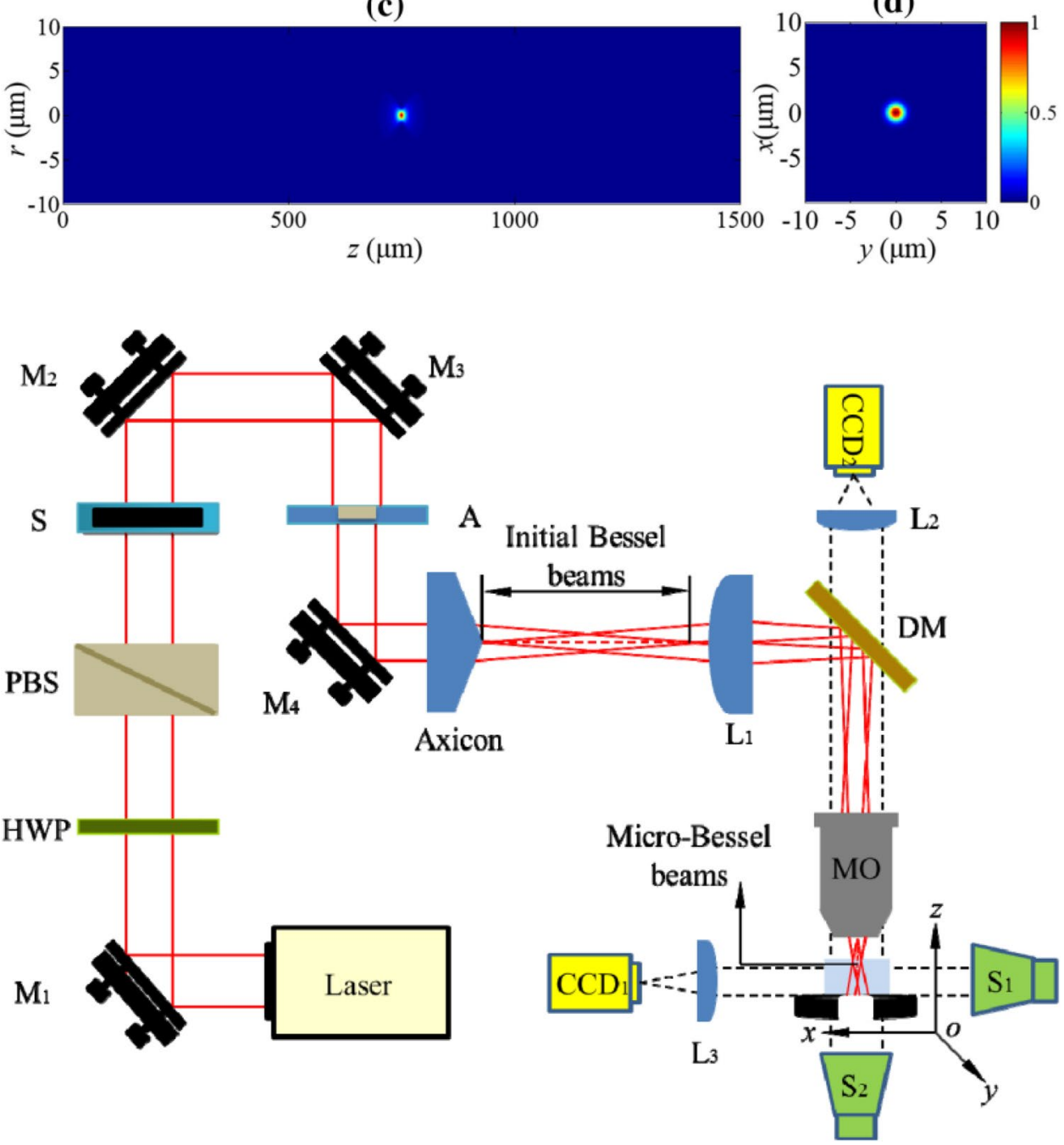

laser. Another choice for Bessel beams generation is the conical lens. Jiang et al. [188] used the combination of flat convex lens and conical lens to form Bessel beam, and fabricated more than 460 high-aspect-ratio microholes on PMMA materials. The schematic diagram of the experimental device is shown in Fig. 48.

The drilling of microholes using the femtosecond laser is a multifactor nonequilibrium complex process, and the processing environments such as vacuum, gas, and liquid greatly affect the microholes [189-192]. The power density of a focused femtosecond laser can easily reach the ionization threshold of air (about $10^{14} \mathrm{~W} / \mathrm{cm}^{2}$ ) [193]. The Kerr effect, due to a strong electric field, will generate laser self-focusing. When drilling in air, a high laser power density exceeding the air ionization threshold can easily produce a large number of free electrons. When the free electron density is greater than $10^{18} / \mathrm{cm}^{3}$, air ionization plasma is formed and the laser is defocused by the plasma. Moreover, in balance with the self-focusing, a series of hotspots are formed, which is also called the filament phenomenon [194, 195]. However, the laser beam in the filament state is unstable in the propagation process and diverges after a certain distance 


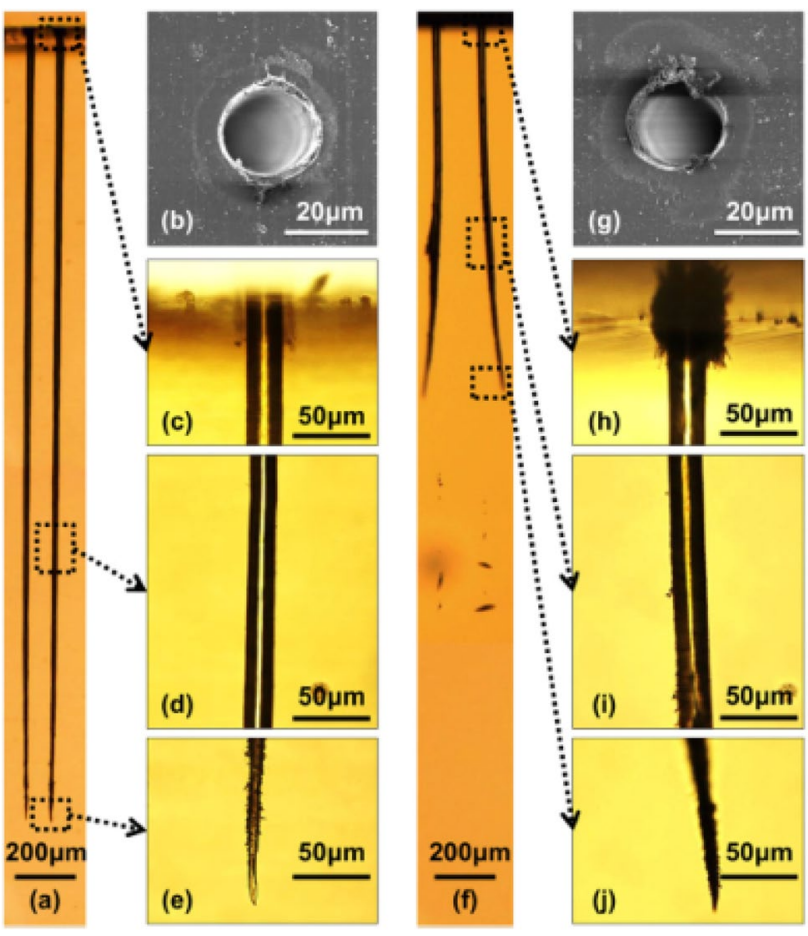

Fig. 49 Comparisons of microhole shapes drilled in vacuum (1 Pa) (a) and air (105 Pa) (f) with 5000 pulses having a pulse energy of $40 \mu \mathrm{J}$. c-e, h-j Magnified transmission optical side view microscopy images that refer to $\mathbf{a}$ and $\mathbf{f}$, respectively. b, $\mathbf{g}$ Top view SEM images of microholes drilled in vacuum and air, respectively [189]

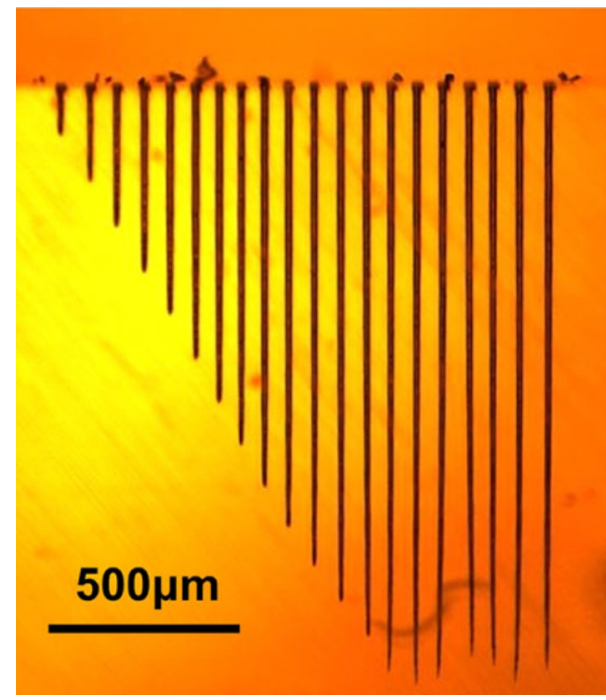

Fig. 50 Microholes drilled in vacuum with number of pulses increasing from 100 to 2000 for a single-pulse energy of $30 \mu \mathrm{J}$ [189]

such that hole bending and ablation saturation occur when drilling deep holes, which can affect the hole taper, inlet diameter, and outlet diameter [184]. In a vacuum environment, the fabricated debris is more easily ejected toward the outside of the material because of the low pressure. Therefore, the recast layer on the surface of the material is smaller $[189,196]$. Figures 49 and 50 show the quality of the holes processed in air and vacuum.

By comparing PMMA materials processed in air and vacuum with high-quality microholes, Xia et al. found that the depth of microholes processed in vacuum increased by two times compared with the depth of microholes processed in air [189]. In addition, a liquid environment can improve the quality of microholes. Li et al. [190] drilled microholes in transparent media using water-assisted methods, which can effectively prevent the influence of the machined morphology on the subsequent laser and help obtain a microchannel structure with a diameter of $4 \mu \mathrm{m}$. The depth of the microchannel exceeds $200 \mu \mathrm{m}$, and the aspect ratio reaches 50:1. However, the debris produced by ablation very easily stays in the hole, which greatly restricts the processing efficiency. To solve these problems, on the basis of liquid-assisted, the efficiency of ablation products discharged from the microholes was accelerated by ultrasonic-assisted method [191]. This method greatly improved the fabrication quality of microholes and increased the processing efficiency to up to $30 \mu \mathrm{m} / \mathrm{s}$. Jiao et al. [192] obtained thinner liquid membranes from volatile liquids to improve the auxiliary effect of water membranes. However, many unsolved problems remain in the liquid-assisted drilling of microholes, such as the loss of laser energy absorbed by water and scattering of laser beams by the liquid and bubbles. Jiang et al. [186] use flying punch method to process PMMA. It can process 100 microholes per second, and complete 251,000 microholes in all in $1 \mathrm{~cm}^{2}$ area in $42 \mathrm{~min}$. The diameter of each hole is about $2 \mu \mathrm{m}$, and the aspect ratio can reach 330:1, as shown in Fig. 51.

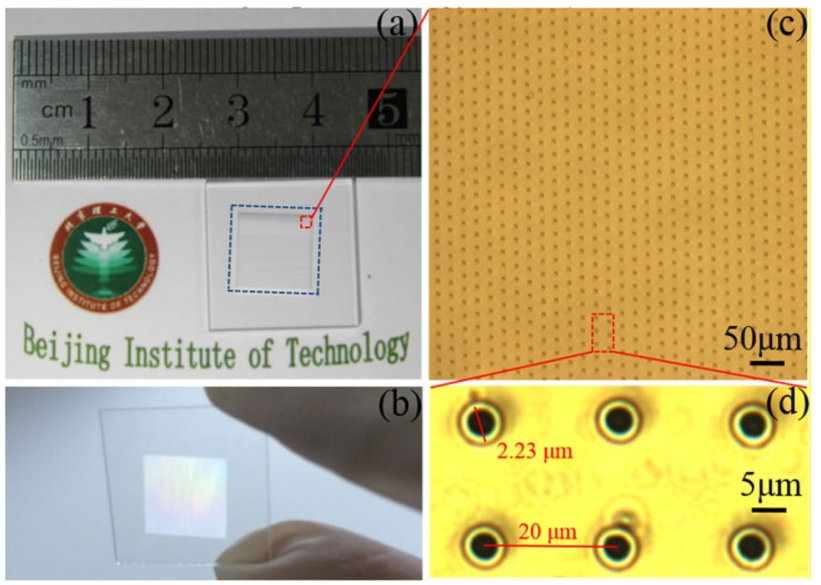

Fig. 51 a, b Group microholes in $1 \mathrm{~cm}^{2}$ area processed on PMMA by flying punch method; $\mathbf{c}$ the local magnified OTM image of a microhole array; $\mathbf{d}$ the diameter and size of each microhole after local amplification and the interval between the microholes [186] 


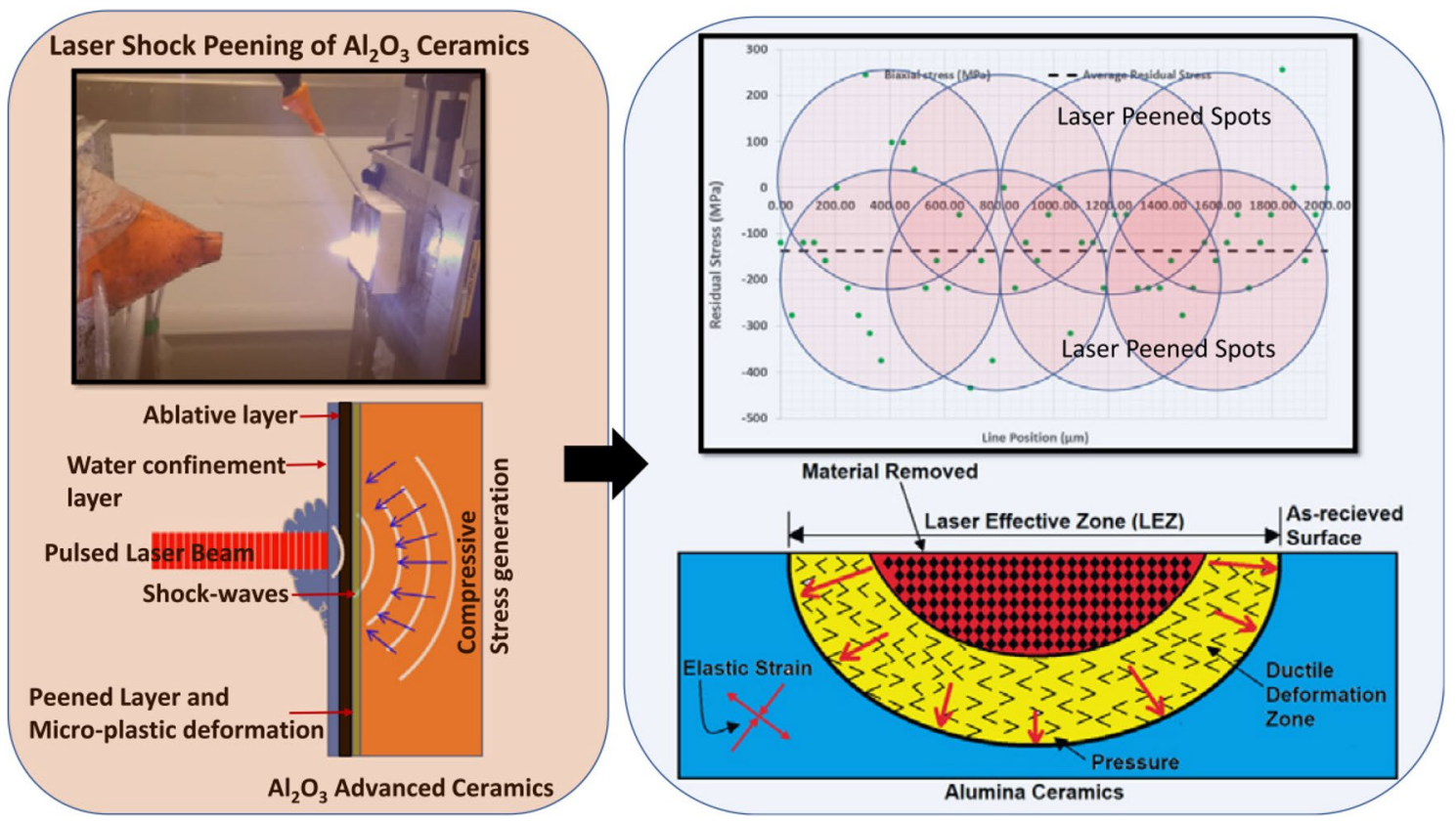

Fig. 52 Laser shock peening on $\mathrm{Al}_{2} \mathrm{O}_{3}$ ceramics [202]

Table 5 Several LSP experiments on various materials

\begin{tabular}{|c|c|c|c|c|c|}
\hline Materials & Pulse duration & Sacrificial layer & Protection layer & Results & Remarks \\
\hline Titanium alloy [204] & $6 \mathrm{~ns}$ & Black tape & Water & $\begin{array}{l}\text { Hardness increased } 20 \mathrm{Hv} \\
\text { with protection layer }\end{array}$ & $\begin{array}{l}\text { Effects of protection layers on } \\
\text { hardness }\end{array}$ \\
\hline AISI 316 stainless steel [205] & $100 \mathrm{fs}$ & Sticky tape & Water & $\begin{array}{l}\text { Deeper penetration with } \\
\text { sacrificial layer }\end{array}$ & $\begin{array}{l}\text { Laser parameters in accord- } \\
\text { ance with the sacrificial } \\
\text { layer thickness }\end{array}$ \\
\hline SUS304 stainless steel [206] & $191 \mathrm{fs}$ & None & Water & $\begin{array}{l}\text { Two times hardness value } \\
\text { increasing }\end{array}$ & $\begin{array}{l}\text { Linear relationship between } \\
\text { hardness and shot number }\end{array}$ \\
\hline Mg alloy [207] & $400 \mathrm{fs}$ & None & None & $\begin{array}{l}\text { Refined micro-structure, } \\
2-3 \mu \mathrm{m} \text { melting depth }\end{array}$ & $\begin{array}{l}\text { Corrosion reduction of } \\
\text { medical-used alloy }\end{array}$ \\
\hline $\begin{array}{l}\text { 7075-T73 aluminum alloy } \\
\text { [208] }\end{array}$ & $130 \mathrm{fs}$ & None & None & $\begin{array}{l}\text { High-density dislocations, } \\
\text { fatigue strength by } 50 \mathrm{MPa} \\
\text { and the fatigue life by } \\
\text { seven times increasing at } \\
\text { most }\end{array}$ & $\begin{array}{l}\text { Hardened with no sacrificial } \\
\text { or protection layer; potential } \\
\text { in vacuum circumstance }\end{array}$ \\
\hline
\end{tabular}

\subsection{Laser Shock Peening}

Laser shock peening (LSP) is a crucial method that is exemplified by the steel strengthening applied in the aerospace industry and other military manufacturing fields [197-199]. Nanosecond laser was commonly used for shock peening [200, 201]. Laser shock peening uses shock waves to deform the specimens with or without protection layers and sacrificial layers. The equipment and schematic diagram of shock wave and effects of laser peening on $\mathrm{Al}_{2} \mathrm{O}_{3}$ ceramics are shown as an example in Fig. 52 [202].

In recent years, the advantages of femtosecond laser shock intensification have attracted much attention than other shock enhancement methods. Femtosecond laser shock peening (FSLSP) can produce higher impact pressure then NSLSP, and thus obtain better hardening effect. However, the lower peening depth of FSLSP couldn't be neglected as well [203]. Table 5 shows the parameters and main results of nanosecond laser shock peening (NSLSP) and femtosecond laser shock peening (FSLSP) on different materials.

According to Fabbro's theory [209] of laser shock wave, the effects of LSP were determined by several key factors like laser power density and the materials of sacrificial layer and protection layer. In 2001, J. Kaspar and A. Luft [203] have compared the differences of LSP efficiency on titanium alloy with or without protection layers and sacrificial 
layers. The results showed the benefit of two layers on strengthening.

Hoppius et al. [205] also used a femtosecond laser to impact AISI 316 stainless steel and compared the change in material hardness after impact at different depths of the laser focus sacrificial layer. When the sacrificial layer was ablated, the hardness of the material increased with increasing laser energy density. However, when the sacrificial layer was removed completely, the hardness decreased as the energy density increased. Such ablative removal of the sacrificial layer by the final laser pulse prevented direct interaction between the material surface and the laser and enhanced the shock peening effect. They also pointed out the lack of peening depth of FSLSP and suggested that FSLSP needed to improve the efficiency of hardening.

Based on Fabbro's theory, the detailed parameters of the shock laser have been investigated. Kawashima et al. [210] used a laser with a wavelength of $800 \mathrm{~nm}$ and pulse width of 120 fs to strengthen 2024-t351 aluminum alloy and studied the effects of different laser energies and spot coverage on the residual stress of the peening material. The dissipation depth of material residual stress was
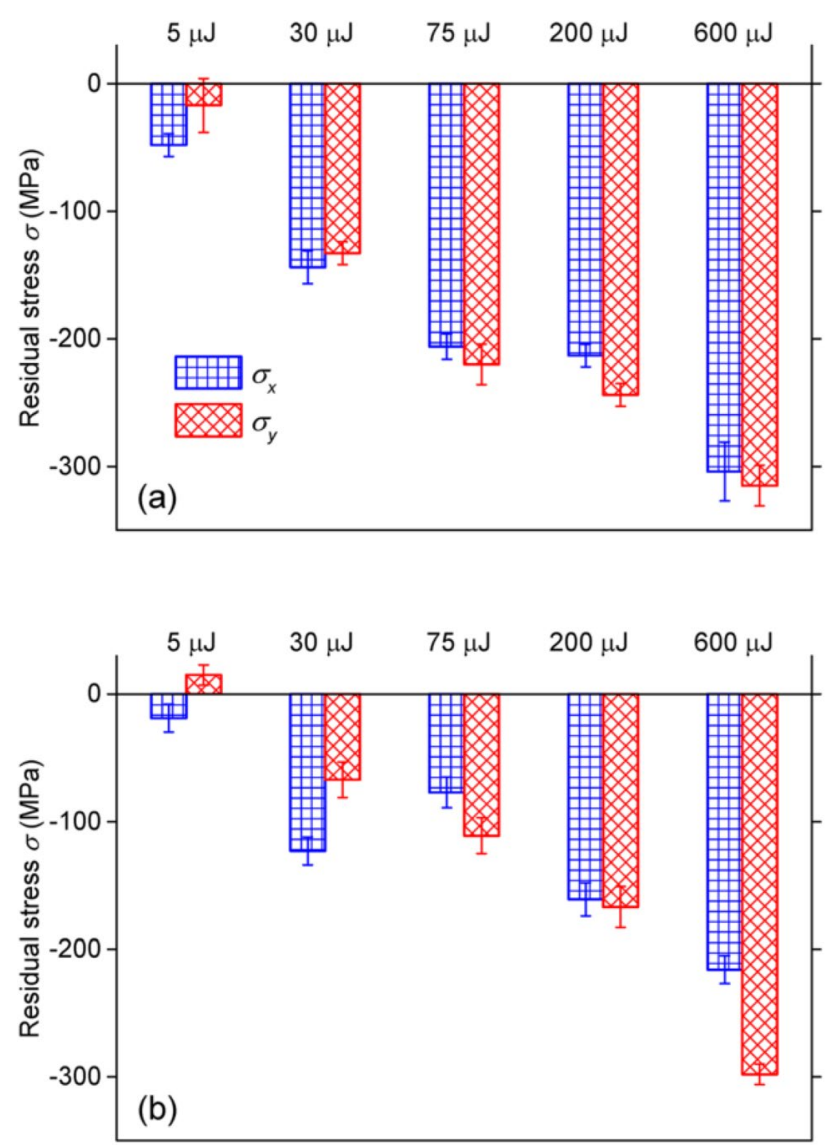

Fig. 53 Residual stress under femtosecond irradiation with coverage of a $692 \%$ and b $2768 \%$ [210] compared when the spot coverage was $692 \%$ and $2768 \%$ and when the laser frequency was 5, 30, 75, 200, and $600 \mu \mathrm{J}$ (Fig. 53). When the laser energy was $600 \mu \mathrm{J}$ and the spot coverage was $2768 \%$, the fatigue life of the material was 38 times higher than that in case of the non-shocked base material.

Majumdar et al. [211] used a 300-fs laser under different parameters to shock $0.4 \% \mathrm{C}$ steel. In his paper, residual stress on the surface was introduced from $140 \mathrm{MPa}$ (tensile) to $-335 \mathrm{MPa}$ (compressive) at most, which doubled the hardness of the steel.

Nevertheless, reports comparing femtosecond laser shock intensification with other strengthening methods of the same material under the same conditions are inadequate and urgently improvement in FSLSP depth of shock zone is still needed. Potential advantages of using femtosecond lasers for material strengthening and the deep correlations between parameters and hardening effects need to be elucidated further, as this technology is expected to be used more widely in the future.

\section{Conclusions}

In this paper, we reviewed the development of femtosecond laser micro/nano manufacturing including the related theories, methods, measurements, and applications. In terms of the theory, the first-principles, molecular dynamics, improved two-temperature model, and plasma quantum models were introduced to interpret the mechanism of femtosecond laser-material interactions. Each model has its own limitations in the temporal and spatial dimensions with respect to explaining the mechanisms of the interaction of femtosecond laser with materials. Therefore, a multiscale theoretical model was proposed to completely explain the interaction processes between a femtosecond laser and a material. To verify the theories, the multiscale observation systems including the femtosecond laser pump-probe and continuous imaging technology, time-resolved plasma imaging technology, LIBS, and $\mathrm{CCD} / \mathrm{ICCD}$ imaging technology were developed to visually observe dynamic changes in materials throughout the process in different temporal and spatial scales.

The methods of femtosecond laser fabrication mainly include femtosecond laser direct writing, femtosecond laser time/space shaping, femtosecond laser frequency modulation such as second harmonic generation or OPA, and femtosecond-laser-induced chemical reaction. These fabrication methods have greatly expanded the range of applications of femtosecond laser fabrication and have made it possible to process any material and construct complicated 3D structures. 
Owing to the advantages of femtosecond lasers and the newly developed fabrication methods, femtosecond laser fabrication has become an irreplaceable manufacturing method in many application areas such as the processing of special functional surfaces, biological tissue or cells, and high-aspect-ratio microholes. For example, microholes with aspect ratios greater than 1000:1 have been fabricated on transparent materials. The fabrication of highaspect-ratio microholes on metallic materials remains challenging because of their high reflection rate. The current femtosecond laser fabrication technology is mainly applied to flat or simple curved surfaces. In fact, complex 3D structures can really take advantage of femtosecond laser manufacturing. The development of new methods to further improve the efficiency of femtosecond laser fabrication is necessary for large-area fabrication, such as carbon-based material processing applications. With the continuous development of femtosecond laser technology and further improvements of femtosecond laser fabrication theories and methods like the method of electronic level control and feedback manufacturing, femtosecond laser fabrication is expected to become an effective means of high-quality extreme manufacturing and to move towards large-scale commercial applications.

Acknowledgements This study was supported by the National Natural Science Foundation of China under Grant Nos. 51975054 and 61605140, the National Key R\&D Program of China (2017YFB1104300), and Beijing Institute of Technology Research Fund Program for Young Scholars.

Open Access This article is licensed under a Creative Commons Attribution 4.0 International License, which permits use, sharing, adaptation, distribution and reproduction in any medium or format, as long as you give appropriate credit to the original author(s) and the source, provide a link to the Creative Commons licence, and indicate if changes were made. The images or other third party material in this article are included in the article's Creative Commons licence, unless indicated otherwise in a credit line to the material. If material is not included in the article's Creative Commons licence and your intended use is not permitted by statutory regulation or exceeds the permitted use, you will need to obtain permission directly from the copyright holder. To view a copy of this licence, visit http://creativecommons.org/licenses/by/4.0/.

\section{References}

1. Gattass R, Mazur E (2008) Femtosecond laser micromachining in transparent materials. Nat Photonics 2:219-225

2. Sugioka K, Cheng Y (2014) Ultrafast lasers-reliable tools for advanced materials processing. Light Sci Appl 3(4):e149

3. Kerse C, Kalaycioğlu H, Elahi P et al (2016) Ablation-cooled material removal with ultrafast bursts of pulses. Nature 537(7618):84-88

4. Jiang L, Tsai HL (2005) Repeatable nanostructures in dielectrics by femtosecond laser pulse trains. Appl Phys Lett 87(15):151104
5. Zhao Y, Han Q, Cheng Z, Jiang L, Qu L (2017) Integrated graphene systems by laser irradiation for advanced devices. Nano Today 12:14-30

6. Tan D, Sharafudeen KN, Yue Y, Qiu J (2016) Femtosecond laser induced phenomena in transparent solid materials: fundamentals and applications. Prog Mater Sci 76:154-228

7. Yin K, Duan JA, Sun X, Wang C, Luo Z (2015) Formation of superwetting surface with line-patterned nanostructure on sapphire induced by femtosecond laser. Appl Phys A 119(1):69-74

8. Wang C, Luo Z, Duan JA, Jiang L, Sun XY, Hu YW, Zhou JY, Lu YF (2015) Adjustable annular rings of periodic surface structures induced by spatially shaped femtosecond laser. Laser Phys Lett 12(5):056001

9. Wang AD, Jiang L, Li XW, Liu Y, Dong XZ, Qu LT, Duan XM, Lu YF (2015) Mask-free patterning of high-conductivity metal nanowires in open air by spatially modulated femtosecond laser pulses. Adv Mater 27(40):6238-6243

10. Fan PX, Bai BF, Long JY, Jiang DF, Jin GF, Zhang HJ, Zhong ML (2015) Broadband high-performance infrared antireflection nanowires facilely grown on ultrafast laser structured $\mathrm{Cu}$ surface. Nano Lett 15(9):5988-5994

11. Cao YY, Takeyasu N, Tanaka T, Duan XM, Kawata S (2009) 3D metallic nanostructure fabrication by surfactant-assisted multiphoton-induced reduction. Small 5(10):1144-1148

12. Balling P, Schou J (2013) Femtosecond-laser ablation dynamics of dielectrics: basics and applications for thin films. Rep Prog Phys 76(3):036502

13. Eaton SM, Cerullo G, Osellame R (2012) Fundamentals of femtosecond laser modification of bulk dielectrics. Femtosecond Laser Micromach 2012:3-18

14. Stoian R, Rosenfeld A, Ashkenasi D, Hertel IV, Bulgakova NM, Campbell EEB (2002) Surface charging and impulsive ion ejection during ultrashort pulsed laser ablation. Phys Rev Lett 88(9):097603

15. Wang C, Jiang L, Wang F, Li X, Yuan YP, Tsai HL (2011) First-principles calculations of the electron dynamics during femtosecond laser pulse train material interactions. Phys Lett A 375(36):3200-3204

16. Su GS, Wang F, Jiang L, Zhang XQ, Su XX, Qu LT, Lu YF (2017) Ultrafast response of dielectric properties of monolayer phosphorene to femtosecond laser. J Appl Phys 121(17):173105

17. Girifalco LA, Weizer VG (1959) Application of the Morse potential function to cubic metals. Phys Rev 114(3):687

18. Li X, Jiang L (2012) Size distribution control of metal nanoparticles using femtosecond laser pulse train: a molecular dynamics simulation. Appl Phys A 109(2):367-376

19. Yuan YP, Jiang L, Li X, Wang C, Xiao H, Lu YF, Tsai HL (2012) Formation mechanisms of sub-wavelength ripples during femtosecond laser pulse train processing of dielectrics. J Phys D Appl Phys 45(17): 175301

20. Yuan YP, Jiang L, Li X, Wang C, Lu YF (2012) Adjustment of ablation shapes and subwavelength ripples based on electron dynamics control by designing femtosecond laser pulse trains. J Appl Phys 112(10):103103

21. Yuan YP, Jiang L, Li X, Wang C, Qu LT, Lu YF (2013) Simulation of rippled structure adjustments based on localized transient electron dynamics control by femtosecond laser pulse trains. Appl Phys A 111(3):813-819

22. Anisimov SI, Kapeliovich BL, Perelman TL (1974) Electron emission from metal surfaces exposed to ultrashort laser pulses. J Exp Theor Phys 66(2):375-377

23. Jiang L, Tsai HL (2005) Improved two-temperature model and its application in ultrashort laser heating of metal films. J Heat Transfer 127(10):1167-1173

24. Tsai H, Jiang L (2005) Fundamentals of energy cascade during ultrashort laser-material interactions. Proc SPIE 5713:343-357 
25. Waldecker L, Bertoni R, Ernstorfer R, Vorberger J (2016) Electron-phonon coupling and energy flow in a simple metal beyond the two-temperature approximation. Phys Rev X 6(2):021003

26. Ahmadi TS, Logunov SL, El-Sayed MA (1996) Picosecond dynamics of colloidal gold nanoparticles. J Phys Chem 100(20):8053-8056

27. Wellershoff SS, Hohlfeld J, Güdde J, Matthias E (1999) The role of electron-phonon coupling in femtosecond laser damage of metals. Appl Phys A 69(1):S99-S107

28. Rethfeld B, Kaiser A, Vicanek M, Simon G (2002) Ultrafast dynamics of nonequilibrium electrons in metals under femtosecond laser irradiation. Phys Rev B 65(21):214303

29. Bovensiepen U (2007) Coherent and incoherent excitations of the Gd (0001) surface on ultrafast timescales. J Phys: Condens Matter 19(8):083201

30. Zhang Y, Chen JK (2007) Melting and resolidification of gold film irradiated by nano- to femtosecond lasers. Appl Phys A 88(2):289-297

31. Atxitia U, Chubykalo-Fesenko O, Walowski J, Mann A, Münzenberg M (2010) Evidence for thermal mechanisms in laserinduced femtosecond spin dynamics. Phys Rev B 81(17):174401

32. Scotognella F, Della VG, Srimath KA, Dorfs D et al (2011) Plasmon dynamics in colloidal $\mathrm{Cu}_{2-x}$ Se nanocrystals. Nano Lett 11(11):4711-4717

33. Giret Y, Gellé A, Arnaud B (2011) Entropy driven atomic motion in laser-excited bismuth. Phys Rev Lett 106(15):155503

34. Derrien TJY, Krüger J, Itina TE, Höhm S, Rosenfeld A, Bonse J (2013) Rippled area formed by surface plasmon polaritons upon femtosecond laser double-pulse irradiation of silicon. Opt Express 21(24):29643-39655

35. Tsai HL, Jiang L (2008) Multiscale modeling of ultrafast lasermaterial interactions. Appl Lasers Opt LIA 1:755-760

36. Jiang L, Wang AD, Cao Q, Li X, Yu Y, Tan X (2014) Ultrafast laser continuous imaging device and method based on spatial time transform in frequency domain (Patent 201410683514.1)

37. Nakagawa K, Iwasaki A, Oishi Y, Horisaki R, Tsukamoto A, Nakamura A, Hirosawa K, Liao H, Ushida T, Goda K, Kannari F (2014) Sequentially timed all-optical mapping photography (STAMP). Nat Photonics 8(9):695

38. Pan CJ, Jiang L, Wang QS, Sun JY, Wang GY, Lu YF (2018) Temporal-spatial measurement of electron relaxation time in femtosecond laser induced plasma using two-color pump-probe imaging technique. Appl Phys Lett 112(19):191101

39. Kubel M, Dube Z, Naumov AY, Villeneuve DM, Corkum PB, Staudte A (2019) Spatiotemporal imaging of valence electron motion. Nat Commun 10(1):1042

40. Wenz J, Döpp A, Khrennikov K, Schindler S, Gilljohann MF, Ding H, Götzfried J, Buck A, Xu J, Heigoldt M, Helml W (2019) Dual-energy electron beams from a compact laser-driven accelerator. Nat Photonics 13(4):263

41. Wang MM, Wang SM, Cao ZT, Wang P, Wang C (2015) Investigation of double-pulse femtosecond laser induced breakdown spectroscopy of polymethyl methacrylate (PMMA). Laser-Based Micro- Nanoprocess IX 9351:93511Q

42. Wang XW, Chen AM, Sui LZ, Wang Y, Zhang D, Li SY, Jiang YF, Jin MX (2018) Temperature dependence of emission intensity in femtosecond laser-induced Ge plasma. J Anal At Spectrom 33(2):168-174

43. Cheng L, Park JS, Hou H, Zorba V, Chen G, Richardson T, Cabana J, Russo R, Doeff M (2014) Effect of microstructure and surface impurity segregation on the electrical and electrochemical properties of dense $\mathrm{Al}$-substituted $\mathrm{Li}_{7} \mathrm{La}_{3} \mathrm{Zr}_{2} \mathrm{O}_{12}$. J Mater Chem 2(1):172-181

44. Rawlings C, Ryu YK, Rüegg M, Lassaline N, Schwemmer C, Duerig U, Knoll AW, Durrani Z, Wang C, Liu D, Jones ME (2018) Fast turnaround fabrication of silicon point-contact quantum-dot transistors using combined thermal scanning probe lithography and laser writing. Nanotechnology 29(50):505302

45. Cheng Y, Sugioka K, Midorikawa K, Masuda M, Toyoda K, Kawachi M, Shihoyama K (2003) Control of the cross-sectional shape of a hollow microchannel embedded in photostructurable glass by use of a femtosecond laser. Opt Lett 28(1):55-57

46. Hnatovsky C, Taylor RS, Simova E, Rajeev PP, Rayner DM, Bhardwaj VR, Corkum PB (2006) Fabrication of microchannels in glass using focused femtosecond laser radiation and selective chemical etching. Appl Phys A 84(1-2):47-61

47. Yong JL, Huo JL, Yang Q, Chen F, Fang Y, Wu XJ, Liu L, Lu XY, Zhang JZ, Hou X (2018) Femtosecond laser direct writing of porous network microstructures for fabricating super-slippery surfaces with excellent liquid repellence and anti-cell proliferation. Adv Mater Interfaces 5(7):1701479

48. Florea C, Winick KA (2003) Fabrication and characterization of photonicdevices directly written in glass using femtosecond laser pulses. J Lightwave Technol 21(1):246

49. Kravtsov K, Prucnal PR (2009) Ultrashort optical pulse detection for high-speed asynchronous optical CDMA networks. J Lightwave Technol 27(18):4069-4075

50. Yoo SJ, Heritage JP, Hernandez VJ, Scott RP, Cong W, Fontaine NK, Broeke RG, Cao J, Seo SW, Baek JH, Soares FM (2007) Spectral phase encoded time spread optical code division multiple access technology for next generation communication networks. J Opt Netw 6(10):1210-1227

51. Tereshchenko SA, Danilov AA, Dolgushin SA, Titenok SA (2011) Determination of optical characteristics of biological tissues by temporal distribution of an ultrashort laser pulse passed through homogeneous scattering layer. Opt Spectrosc 110(4):652

52. Hagemann F, Gause O, Wöste L, Siebert T (2013) Supercontinuum pulse shaping in the few-cycle regime. Opt Express 21(5):5536-5549

53. Jiang L, Wang AD, Li B, Cui TH, Lu YF (2018) Electrons dynamics control by shaping femtosecond laser pulses in micro/ nanofabrication: modeling, method, measurement and application. Light: Sci Appl 7(2):17134

54. Wang AD, Jiang L, Li XW, Wang Z, Du K, Lu YF (2018) Simple and robust generation of ultrafast laser pulse trains using polarization-independent parallel-aligned thin films. Opt Laser Technol 101:298-303

55. Zhang JY, Xu Z, Kong Y, Yu C, Wu Y (1998) Highly efficient, widely tunable, $10-\mathrm{Hz}$ parametric amplifier pumped by frequency-doubled femtosecond Ti: sapphire laser pulses. Appl Opt 37(15):3299-3305

56. Gale GM, Gallot G, Hache F, Sander R (1997) Generation of intense highly coherent femtosecond pulses in the mid infrared. Opt Lett 22(16):1253-1255

57. Kobayashi T, Shirakawa A, Fuji T (2001) Sub-5-fs transformlimited visible pulse source and its application to real-time spectroscopy. IEEE J Sel Top Quantum Electron 7(4):525-538

58. Zewail AH (2000) Femtochemistry: atomic-scale dynamics of the chemical bond. J Phys Chem A 104(24):5660-5694

59. Petruczok CD, Gleason KK (2012) Initiated chemical vapor deposition-based method for patterning polymer and metal microstructures on curved substrates. Adv Mater 24(48):6445-6450

60. Yang X, Li W, Zhang DH (2012) Subwavelength lithography using metallic grating waveguide heterostructure. Appl Phys A 107(1):123-126

61. Toriyama S, Mizeikis V, Ono A (2019) Fabrication of silver nano-rings using photo-reduction induced by femtosecond pulses. Appl Phys Express 12(1):015004

62. Cônsoli PM, Otuka AJ, Balogh DT, Mendonça CR (2018) Feature size reduction in two-photon polymerization by optimizing resin composition. J Polym Sci, Part B: Polym Phys 56(16):1158-1163 
63. Xia H, Wang J, Tian Y, Chen QD, Du XB, Zhang YL, He Y, Sun HB (2010) Ferrofluids for processing of remotely controllable micro-nanomachines by two-photonpolymerization. Adv Mater 22(29):3204-3207

64. Zhao MJ, Hu J, Jiang L, Zhang KH, Liu PJ, Lu YF (2015) Controllable high-throughput high-quality femtosecond laserenhanced chemical etching by temporal pulse shaping based on electron density control. Sci Rep 5:13202

65. George JE, Rodrigues VR, Mathur D, Chidangil S, George SD (2016) Self-cleaning superhydrophobic surfaces with underwater superaerophobicity. Mater Des 100:8-18

66. Liu KS, Jiang L (2011) Bio-inspired design of multiscale structures for function integration. Nano Today 6(2):155-175

67. Wang B, Liang WX, Guo ZG, Liu WM (2015) Biomimetic super-lyophobic and super-lyophilic materials applied for oil/ water separation: a new strategy beyond nature. Chem Soc Rev 44(1):336-361

68. Zhou XY, Zhang ZZ, Xu XH, Guo F, Zhu XT, Men XH, Ge B (2013) Robust and durable superhydrophobic cotton fabrics for oil/water separation. ACS Appl Mater Interfaces 5(15):7208-7214

69. Crick CR, Gibbins JA, Parkin IP (2013) Superhydrophobic polymer-coated copper-mesh; membranes for highly efficient oil-water separation. J Mater Chem A 1(19):5943-5948

70. Cao L, Jones AK, Sikka VK, Wu J, Gao D (2009) Anti-icing superhydrophobic coatings Langmuir 25(21):12444-12448

71. Farhadi S, Farzaneh M, Kulinich SA (2011) Anti-icing performance of superhydrophobic surfaces. Appl Surf Sci 257(14):6264-6269

72. Dong HY, Cheng MJ, Zhang YJ, Wei H, Shi F (2013) Extraordinary drag-reducing effect of a superhydrophobic coating on a macroscopic model ship at high speed. J Mater Chem A 1(19):5886-5891

73. McHale G, Newton MI, Shirtcliffe NJ (2010) Immersed superhydrophobic surfaces: Gas exchange, slip and drag reduction properties. Soft Matter 6(4):714-719

74. Barthlott W, Neinhuis C (1997) Purity of the sacred lotus, or escape from contamination in biological surfaces. Planta 202(1):1-8

75. Yong JL, Chen F, Yang Q, Huo JL, Hou X (2017) Superoleophobic surfaces. Chem Soc Rev 46(14):4168-4217

76. Feng L, Li S, Li H et al (2002) Super-hydrophobic surfaces: from natural to artificial. Adv Mater 14(24):1857-1860

77. Genzer J, Efimenko K (2006) Recent developments in superhydrophobic surfaces and their relevance to marine fouling: a review. Biofouling 22(5):339-360

78. Moradi S, Kamal S, Englezos P, Hatzikiriakos SG (2013) Femtosecond laser irradiation of metallic surfaces: effects of laser parameters on superhydrophobicity. Nanotechnology 24(41):415302

79. Wenzel RN (1936) Resistance of solid surfaces to wetting by water. Ind Eng Chem 28(8):988-994

80. Cassie ABD, Baxter S (1944) Wettability of porous surfaces. Trans Faraday Soc 40:546-551

81. Long JY, Fan PX, Gong DW, Jiang DF, Zhang HJ, Li L, Zhong ML (2015) Superhydrophobic surfaces fabricated by femtosecond laser with tunable water adhesion: from lotus leaf to rose petal. ACS Appl Mater Interfaces 7(18):9858-9865

82. Yan YY, Gao N, Barthlott W (2011) Mimicking natural superhydrophobic surfaces and grasping the wetting process: a review on recent progress in preparing superhydrophobic surfaces. Adv Coll Interface Sci 169(2):80-105

83. Barberoglou M, Zorba V, Stratakis E, Spanakis E, Tzanetakis P, Anastasiadis SH, Fotakis C (2009) Bio-inspired water repellent surfaces produced by ultrafast laser structuring of silicon. Appl Surf Sci 255(10):5425-5429

84. Yong JL, Yang Q, Chen F et al (2013) Stable superhydrophobic surface with hierarchical mesh-porous structure fabricated by a femtosecond laser. Appl Phys A 111(1):243-249

85. Yoon TO, Shin HJ, Jeoung SC, Park YI (2008) Formation of superhydrophobic poly (dimethysiloxane) by ultrafast laserinduced surface modification. Opt Express 16(17):12715-12725

86. Sugioka K, Cheng Y (2014) Femtosecond laser three-dimensional micro-and nanofabrication. Appl Phys Rev 1(4):041303

87. Ahsan MS, Dewanda F, Lee MS, Sekita H, Sumiyoshi T (2013) Formation of superhydrophobic soda-lime glass surface using femtosecond laser pulses. Appl Surf Sci 265:784-789

88. Lin Y, Han JP, Cai MY et al (2018) Durable and robust transparent superhydrophobic glass surfaces fabricated by a femtosecond laser with exceptional water repellency and thermostability. $\mathbf{J}$ Mater Chem 6(19):9049-9056

89. Shi XS, Li X, Jiang L et al (2015) Femtosecond laser rapid fabrication of large-area rose-like micropatterns on freestanding flexible graphene films. Sci Rep 5:17557

90. Sarbada S, Shin YC (2017) Superhydrophobic contoured surfaces created on metal and polymer using a femtosecond laser. Appl Surf Sci 405:465-475

91. Yong JL, Chen F, Yang Q, Fang Y, Huo JL, Hou X (2015) Femtosecond laser induced hierarchical $\mathrm{ZnO}$ superhydrophobic surfaces with switchable wettability. Chem Commun 51(48):9813-9816

92. Tian DL, Zhang XF, Tian Y et al (2012) Photo-induced wateroil separation based on switchable superhydrophobicity-superhydrophilicity and underwater superoleophobicity of the aligned $\mathrm{ZnO}$ nanorod array-coated mesh films. J Mater Chem 22(37):19652-19657

93. Chu Z, Seeger S (2014) Superamphiphobic surfaces. Chem Soc Rev 43(8):2784-2798

94. Liu TL, Kim CJ (2014) Turning a surface superrepellent even to completely wetting liquids. Science 346(6213):1096-1100

95. Yuan L, Wu TZ, Zhang WJ et al (2014) Engineering superlyophobic surfaces on curable materials based on facile and inexpensive microfabrication. J Mater Chem A 2(19):6952-6959

96. Liu MJ, Wang ST, Wei ZX, Song YL, Jiang L (2009) Bioinspired design of a superoleophobic and low adhesive water/solid interface. Adv Mater 21(6):665-669

97. Whyman G, Bormashenko E (2011) How to make the Cassie wetting state stable. Langmuir 27(13):8171-8176

98. Chu D, Yin K, Dong X, Luo Z, Duan JA (2017) Femtosecond laser fabrication of robust underwater superoleophobic and antioil surface on sapphire. AIP Adv 7(11):115224

99. Li GQ, Zhang Z, Wu PC et al (2016) One-step facile fabrication of controllable microcone and micromolar silicon arrays with tunable wettability by liquid-assisted femtosecond laser irradiation. RSC Advances 6(44):37463-37471

100. Li GQ, Lu Y, Wu PC et al (2015) Fish scale inspired design of underwater superoleophobicmicrocone arrays by sucrose solution assisted femtosecond laser irradiation for multifunctional liquid manipulation. J Mater Chem A 3(36):18675-18683

101. Yin K, Luo Z, Du H, Dong X, Duan JA (2018) Femtosecond laser fabrication of a gradient-wettability mesh for spilled oil crossflow collection. Mater Lett 215:272-275

102. Li GQ, Fan H, Ren FF et al (2016) Multifunctional ultrathin aluminum foil: oil/water separation and particle filtration. J Mater Chem A 4(48): 18832-18840

103. Liu YQ, Han DD, Jiao ZZ et al (2017) Laser-structured Janus wire mesh for efficient oil-water separation. Nanoscale 9(45):17933-17938 
104. Wu SZ, Li CZ, Jiao YL et al (2018) Multifunctional oil-water and immiscible organic liquid separation by micropore arrayed Ti foil. Appl Surf Sci 455:221-226

105. Ye S, Cao Q, Wang QS, Wang TY, Peng Q (2016) A highly efficient, stable, durable, and recyclable filter fabricated by femtosecond laser drilling of a titanium foil for oil-water separation. Sci Rep 6:37591

106. Yin K, Chu DK, Dong XR, Wang C, Duan JA, He J (2017) Femtosecond laser induced robust periodic nanoripple structured mesh for highly efficient oil-water separation. Nanoscale 9(37):14229-14235

107. Yong J, Fang Y, Chen F, Huo J, Yang Q, Bian H, Du G, Hou $X$ (2016) Femtosecond laser ablated durable superhydrophobic PTFE films with micro-through-holes for oil/water separation: separating oil from water and corrosive solutions. Appl Surf Sci 389:1148-1155

108. Mačiulaitis J, Deveikytė M, Rekštytė S, Bratchikov M, Darinskas A, Šimbelytė A, Daunoras G, Laurinavičienė A, Laurinavičius A, Gudas R, Malinauskas M (2015) Preclinical study of SZ2080 material 3D microstructured scaffolds for cartilage tissue engineering made by femtosecond direct laser writing lithography. Biofabrication 7(1):015015

109. König K, Riemann I, Fritzsche W (2001) Nanodissection of human chromosomes with near-infrared femtosecond laser pulses. Opt Lett 26(11):819-821

110. Gong JX, Zhao XM, Xing QR, Li F, Li HY, Chai L, Wang QY, Zheltikov A (2008) Femtosecond laser-induced cell fusion. Appl Phys Lett 92(9):093901

111. Vogel A, Noack J, Hüttman G, Paltauf G (2005) Mechanisms of femtosecond laser nanosurgery of cells and tissues. Appl Phys B 81(8):1015-1047

112. Kohli V, Elezzabi AY, Acker JP (2005) Cell nanosurgery using ultrashort (femtosecond) laser pulses: applications to membrane surgery and cell isolation. Lasers Surg Med Off J Am Soc Laser Med Surg 37(3):227-230

113. Glezer EN, Mazur E (1997) Ultrafast-laser driven micro-explosions in transparent materials. Appl Phys Lett 71(7):882-884

114. Docchio F, Sacchi CA, Marshall J (1986) Experimental investigation of optical breakdown thresholds in ocular media under single pulse irradiation with different pulse durations. Lasers Ophthalmol 1(2):83-93

115. Rudhall AP, Antkowiak M, Tsampoul X, Mazilu M, Metzger NK, Gunn-Moore F, Dholakia K (2012) Exploring the ultrashort pulse laser parameter space for membrane permeabilisation in mammalian cells. Sci Rep 2:858

116. Tirlapur UK, König K (2002) Cell biology: targeted transfection by femtosecond laser. Nature 418(6895):290

117. Stevenson D, Agate B, Tsampoula X, Fischer P, Brown C, Sibbett W, Riches A, GunnMoore F, Dholakia K (2006) Femtosecond optical transfection of cells: viability and efficiency. Opt Express 14(16):7125-7133

118. Guehring T, Urban JP, Cui Z, Tirlapur UK (2008) Noninvasive $3 \mathrm{D}$ vital imaging and characterization of notochordal cells of the intervertebral disc by femtosecond near-infrared two-photon laser scanning microscopy and spatial-volume rendering. Microsc Res Tech 71(4):298-304

119. Schenke-Layland K, Riemann I, Stock UA, König K (2005) Imaging of cardiovascular structures using near-infrared femtosecond multiphoton laser scanning microscopy. J Biomed Opt 10(2):024017

120. Verant P, Serduc R, Coles JA, Farion R, Remy C, van der Sanden B, Vial JC (2004) A method for measuring cerebral blood volume of mouse using multiphoton laser scanning microscopy. Femtosecond Laser Appl Biol 5463:1-13
121. Chen B, Jiang T, Zong W, Chen L, Zhang Z, Wang A (2016) 910 $\mathrm{nm}$ femtosecond $\mathrm{Nd}$-doped fiber laser for in vivo two-photon microscopic imaging. Opt Express 24(15):16544-16549

122. Chu SW, Tai SP, Ho CL, Lin CH, Sun CK (2005) High-resolution simultaneous three-photon fluorescence and third-harmonic-generation microscopy. Microsc Res Tech 66(4):193-197

123. Shimada T, Watanabe W, Matsunaga S, Higashi T, Ishii H, Fukui $\mathrm{K}$, Isobe K, Itoh K (2005) Intracellular disruption of mitochondria in a living HeLa cell with a 76-MHz femtosecond laser oscillator. Opt Express 13(24):9869-9880

124. Watanabe W, Arakawa N, Matsunaga S, Higashi T, Fukui K, Isobe K, Itoh K (2004) Femtosecond laser disruption of subcellular organelles in a living cell. Opt Express 12(18):4203-4213

125. Danilevicius P, Rekštyte S, Gadonas R, Malinauskas M, Balciunas E, Jarasiene R, Baltriukiene D, Bukelskiene V, Kraniauskas A, Sirmenis R (2012) Micro-structured polymer scaffolds fabricated by direct laser writing for tissue engineering. J Biomed Opt 17(8):081405

126. Zhu S, Li H, Yang M, Pang SW (2016) High sensitivity plasmonic biosensor based on nanoimprinted quasi 3D nanosquares for cell detection. Nanotechnology 27(29):295101

127. Boyuan Z, Fengying J, Zhishan H et al (2016) PPreparation of biocompatible protein-hydrogel-based micro-patterns via UV lithography. Chem J Chin Univ-Chin 37(4):715-722

128. Ma ZC, Chen QD, Han B, Liu XQ, Song JF, Sun HB (2015) Measurement of two-photon absorption cross section of metal ions by a mass sedimentation approach. Sci Rep 5:17712

129. Wu D, Wang JN, Niu LG, Zhang XL, Wu SZ, Chen QD, Lee LP, Sun HB (2014) Bioinspired fabrication of high-quality 3D artificial compound eyes by voxel-modulation femtosecond laser writing for distortion-free wide-field-of-view imaging. Adv Opt Mater 2(28):751-758

130. Kaehr B, Shear JB (2007) Mask-directed multiphoton lithography. J Am Chem Soc 129(7):1904-1905

131. Spivey EC, Ritschdorff ET, Connell JL, McLennon CA, Schmidt CE, Shear JB (2013) Multiphoton lithography of unconstrained three-dimensional protein microstructures. Adv Func Mater 23(3):333-339

132. Bhat WA (2018) Bridging data-capacity gap in big data storage. Future Generation Comput Syst 87:538-548

133. Dai L, Tan C, Wang L, Han XB, Liu CR, Xu YH (2018) Investigation on nonvolatile holographic storage properties in Hf: Ru: Fe: $\mathrm{LiNbO}_{3}$ crystals as a function of Li composition. J Alloy Compd 753:407-411

134. Li ZH, Zhang HL, Li Q, Wang YG, Shao JB, Xu XM, Shi L (2009) Study on holographic characters of two-wavelength sensitive water-resisting photopolymer. J Optoelectron Laser 3:19

135. Fang C, Zhang Y, Zhu H (2012) Optimizing the optical field distribution of near-field SIL optical storage system using fivezone binary phase filters. Opt Commun 285(13-14):3042-3045

136. Glezer EN, Milosavljevic M, Huang L, Finlay RJ, Her TH, Callan JP, Mazur E (1996) Three-dimensional optical storage inside transparent materials. Opt Lett 21(24):2023-2025

137. Zhang J, Gecevičius M, Beresna M, Beresna M, Kazansky PG (2014) Seemingly unlimited lifetime data storage in nanostructured glass. Phys Rev Lett 112(3):033901

138. Strickler JH, Webb WW (1991) Three-dimensional optical data storage in refractive media by two-photon point excitation. Opt Lett 16(22):1780-1782

139. Zhou Y, Han ST, Chen X, Wang F, Tang YB, Roy VAL (2014) An upconverted photonic nonvolatile memory. Nat Commun $5: 4720$

140. Zijlstra P, Chon JWM, Gu M (2009) Five-dimensional optical recording mediated by surface plasmons in gold nanorods. Nature 459(7245):410 
141. Royon A, Bourhis K, Bellec M, Papon G, Bousquet B, Deshayes Y, Cardinal T, Canioni L (2010) Silver clusters embedded in glass as a perennial high capacity optical recording medium. Adv Mater 22(46):5282-5286

142. Klyukin D, Silvennoinen M, Krykova V, Svirko Y, Sidorov A, Nikonorov N (2017) Fluorescent clusters in chloride photothermo-refractive glass by femtosecond laser bleaching of $\mathrm{Ag}$ nanoparticles. Opt Express 25(11):12944-12951

143. Alexey SL, Sergey SF, Andrey GO, Sergey VL, Alexey MV, Alexander AS, Georgiy YS, Kseniya IP, Ivan SG, Vladimir NS (2018) Multilevel data writing in nanoporous glass by a few femtosecond laser pulses. Appl Opt 57(4):978-982

144. Cerkauskaite A, Drevinskas R, Solodar A, Abdulhalim I, Kazansky PG (2017) Form-birefringence in ITO thin films engineered by ultrafast laser nanostructuring. ACS Photonics 4(11):2944-2951

145. Tan CL, Cao XH, Wu XJ, He QY, Yang J, Zhang X, Chen JZ, Zhao W, Han SK, Nam G, Sindoro M, Zhang H (2017) Recent advances in ultrathin two-dimensional nanomaterials. Chem Rev 117(9):6225-6331

146. Hong JY, Jang J (2012) Micropatterning of graphene sheets: recent advances in techniques and applications. J Mater Chem 22(17):8179-8191

147. Zhou Y, Loh KP (2010) Making patterns on graphene. Adv Mater 22(32):3615-3620

148. Wang JN, Shao RQ, Zhang L, Guo L, Jiang HB, Lu DX, Sun HB (2012) Biomimetic graphene surfaces with superhydrophobicity and iridescence. Chem Asian J 7(2):301-304

149. Lorenzoni M, Brandi F, Dante S, Giugni A, Torre B (2013) Simple and effective graphene laser processing for neuron patterning application. Sci Rep 3:1954

150. Wang XL, Shi GQ (2015) Flexible graphene devices related to energy conversion and storage. Energy Environ Sci 8(3):790-823

151. Liang Y, Zhao F, Cheng Z, Deng Y, Xiao Y, Cheng H, Zhang P, Huang Y, Shao H, Qu L (2018) Electric power generation via asymmetric moisturizing of graphene oxide for flexible, printable and portable electronics. Energy Environ Sci 11(7):1730-1735

152. Gil-Villalba A, Meyer R, Giust R, Rapp L, Billet C, Courvoisier F (2018) Single shot femtosecond laser nano-ablation of CVD monolayer graphene. Sci Rep 8(1):14601

153. Yoo JH, Park JB, Ahn S, Grigoropoulos CP (2013) Laser-induced direct graphene patterning and simultaneous transferring method for graphene sensor platform. Small 9(24):4269-4275

154. Stöhr RJ, Kolesov R, Xia K, Wrachtrup J (2011) All-optical highresolution nanopatterning and $3 \mathrm{D}$ suspending of graphene. ACS Nano 5(6):5141-5150

155. Dong XX, Liu SB, Song HY, Gu P (2017) Growth of large-area, few-layer graphene by femtosecond pulsed laser deposition with double-layer Ni catalyst. J Mater Sci 52(4):2060-2065

156. Cheng C, He R, Romero C, Vazquez de Aldana JR, Chen F (2017) Spontaneous micro-modification of single-layer graphene induced by femtosecond laser irradiation. Appl Phys Lett 111(24):241901

157. Tan DZ, Zhou SF, Xu BB, Chen P, Shimotsuma Y, Miura K, Qiu JR (2013) Simple synthesis of ultra-small nanodiamonds with tunable size and photoluminescence. Carbon 62:374-381

158. Li CH, Kang XL, Zhu QH, Zheng WG (2017) Effects of ultraviolet nanosecond laser irradiation on structural modification and optical transmission of single layer graphene. Appl Surf Sci 398:89-96

159. Tan DZ, Liu XF, Dai Y, Ma GH, Meunier M, Qiu JR (2015) A universal photochemical approach to ultra-small, welldispersed nanoparticle/reduced graphene oxide hybrids with enhanced nonlinear optical properties. Adv Opt Mater 3(6):836-841

160. Sahin R, Simsek E, Akturk S (2014) Nanoscale patterning of graphene through femtosecond laser ablation. Appl Phys Lett 104(5):053118

161. Zhang Y, Guo L, Wei S, He Y, Xia H, Chen Q, Sun H, Xiao F (2010) Direct imprinting of microcircuits on graphene oxides film by femtosecond laser reduction. Nano Today 5(1):15-20

162. Kang S, Evans CC, Shukla S, Reshef O, Mazur E (2018) Patterning and reduction of graphene oxide using femtosecondlaser irradiation. Opt Laser Technol 103:340-345

163. Nakamura T, Nishina Y, Sato S (2017) Chemical surface modification of graphene oxide by femtosecond laser pulse irradiation in aqueous suspensions. J Mater Sci 52(2):749-759

164. Yu Y, Si J, Yan L, Li M, Hou X (2019) Enhanced nonlinear absorption and ultrafast carrier dynamics in graphene/gold nanoparticles nanocomposites. Carbon 148:72-79

165. Baheri S, Tabrizi SA, Jubran BA (2008) Film cooling effectiveness from trenched shaped and compound holes. Heat Mass Transf 44(8):989-998

166. Beck T (2011) Laser drilling in gas turbine blades: shaping of holes in ceramic and metallic coatings. Laser Tech $\mathrm{J}$ $8(3): 40-43$

167. Lundgren EH, Forsman AC, Hoppe ML, Moreno KA, Nikroo A (2007) Fabrication of pressurized $2 \mathrm{~mm}$ beryllium targets for ICF experiments. Fusion Sci Technol 51(4):576-580

168. Lundgren EH, Forsman AC (2009) Laser forming of shaped fill holes in beryllium targets for inertial confinement fusion experiments. Fusion Sci Technol 55(3):325-330

169. Wang Y, Wang DN, Yang M, Hong W, Lu P (2009) Refractive index sensor based on a microhole in single-mode fiber created by the use of femtosecond laser micromachining. Opt Lett 34(21):3328-3330

170. Liu S, Jin L, Jin W, Wang D, Liao C, Wang Y (2010) Structural long period gratings made by drilling micro-holes in photonic crystal fibers with a femtosecond infrared laser. Opt Express 18(6):5496-5503

171. Wang X, Huang Z, Zhang W, Kuti OA, Nishida K (2011) Effects of ultra-high injection pressure and micro-hole nozzle on flame structure and soot formation of impinging diesel spray. Appl Energy 88(5):1620-1628

172. Schaffer CB, Brodeur A, Mazur E (2001) Laser-induced breakdown and damage in bulk transparent materials induced by tightly focused femtosecond laser pulses. Meas Sci Technol 12(11): 1784

173. Harzic RL, Huot N, Audouard E, Jonin C, Laporte P (2002) Comparison of heat-affected zones due to nanosecond and femtosecond laser pulses using transmission electronic microscopy. Appl Phys Lett 80(21):3886-3888

174. Kautek W, Krüger J, Lenzner M, Sartania S, Spielmann C, Krausz F (1996) Laser ablation of dielectrics with pulse durations between 20 fs and 3 ps. Appl Phys Lett 69(21):3146-3148

175. Zheng ZP, Lin JK, Huang FY, Yan BH (2008) Improving the machining efficiency in electrochemical discharge machining $(\mathrm{ECDM})$ microhole drilling by offset pulse voltage. J Micromech Microeng 18(2):025014

176. Zhang H, Di J, Zhou M, Yan Y (2014) A comparison in laser precision drilling of stainless steel 304 with nanosecond and picosecond laser pulses. Chin J Mech Eng 27(5):972-977

177. Zhang HY, Di JK, Zhou M, Yan Y, Wang R (2015) An investigation on the hole quality during picosecond laser helical drilling of stainless steel 304. Appl Phys A Mater Sci Process $119(2): 745-752$

178. Tönshoff HK, Momma C, Ostendorf A, Nolte S, Kamlage G (2000) Microdrilling of metals with ultrashort laser pulses. J Laser Appl 12(1):23-27 
179. Wang QX, Luo SZ, Chen Z, Qi HX, Deng JN, Hu Z (2016) Drilling of aluminum and copper films with femtosecond doublepulse laser. Opt Laser Technol 80:116-124

180. Shah L, Tawney J, Richardson M, Richardson K (2001) Femtosecond laser deep hole drilling of silicate glasses in air. Appl Surf Sci 183(3-4):151-164

181. Nakashima S, Sugioka K, Midorikawa K (2010) Enhancement of resolution and quality of nano-hole structure on GaN substrates using the second-harmonic beam of near-infrared femtosecond laser. Appl Phys A 101(3):475-481

182. Eaton SM, Zhang H, Herman PR, Yoshino F, Shah L, Bovatsek J, Arai AY (2005) Heat accumulation effects in femtosecond laserwritten waveguides with variable repetition rate. Opt Express 13(12):4708-4716

183. Jiang L, Liu PJ, Yan XL, Leng N, Xu CC, Xiao H, Lu YF (2012) High-throughput rear-surface drilling of microchannels in glass based on electron dynamics control using femtosecond pulse trains. Opt Lett 37(14):2781-2783

184. Jiang L, Fang JQ, Cao Q, Zhang KH, Wang P, Yu YW, Huang Q, Lu YF (2014) Femtosecond laser high-efficiency drilling of high-aspect-ratio microholes based on free-electron-density adjustments. Appl Opt 53(31):7290-7295

185. Jiang L, Tsai HL (2007) Modeling of ultrashort laser pulsetrain processing of metal thin films. Int $\mathbf{J}$ Heat Mass Transf 50(17-18):3461-3470

186. Xie Q, Li XW, Jiang L, Xia B, Yan XL, Zhao W, Lu YF (2016) High-aspect-ratio, high-quality microdrilling by electron density control using a femtosecond laser Bessel beam. Appl Phys A 122(2): 136

187. Bhuyan MK, Velpula PK, Colombier JP, Olivier T, Faure N, Stoian R (2014) Single-shot high aspect ratio bulk nanostructuring of fused silica using chirp-controlled ultrafast laser Bessel beams. Appl Phys Lett 104(2):021107

188. Zhao W, Li XW, Xia B, Yan XL, Jiang L (2014) Single-pulse femtosecond laser Bessel beams drilling of high-aspect-ratio microholes based on electron dynamics control. Int Symp Optoelectron Technol Appl 2014: Adv Display Technol Nonimaging Opt Eff Des Illum Solar Conc 9296:92960Q

189. Xia B, Jiang L, Li XW, Yan XL, Zhao WW, Lu YF (2015) High aspect ratio, high-quality microholes in PMMA: a comparison between femtosecond laser drilling in air and in vacuum. Appl Phys A 119(1):61-68

190. Li Y, Itoh K, Watanabe W, Yamada K, Kuroda D, Nishii J, Jiang YY (2001) Three-dimensional hole drilling of silica glass from the rear surface with femtosecond laser pulses. Opt Lett 26(23):1912-1914

191. Hwang DJ, Choi TY, Grigoropoulos CP (2004) Liquid-assisted femtosecond laser drilling of straight and three-dimensional microchannels in glass. Appl Phys A 79(3):605-612

192. Jiao LS, Ng EYK, Wee LM, Zheng HY (2011) Role of volatile liquids in debris and hole taper angle reduction during femtosecond laser drilling of silicon. Appl Phys A 104(4):1081-1084

193. Couairon A, Mysyrowicz A (2007) Femtosecond filamentation in transparent media. Phys Rep 441(2-4):47-189

194. Kandidov VP, Kosareva OGGE, Koltun AA (2003) Nonlinearoptical transformation of a high-power femtosecond laser pulse in air. Quantum Electron 33(1):69
195. Shah L, Tawney J, Richardson K (2004) Self-focusing during femtosecond micromachining of silicate glasses. IEEE J Quantum Electron 40(1):57-68

196. Wynne AE, Stuart BC (2003) Rate dependence of shortpulse laser ablation of metals in air and vacuum. Appl Phys A 76(3):373-378

197. Gujba A, Medraj M (2014) Laser peening process and its impact on materials properties in comparison with shot peening and ultrasonic impact peening. Materials 7(12):7925-7974

198. Zhou LC, He WF, Luo SH, Long CB, Wang C, Nie XF, He GY, Shen XJ, Li YH (2016) Laser shock peening induced surface nanocrystallization and martensite transformation in austenitic stainless steel. J Alloy Compd 655:66-70

199. Yu HW, Li XY, Hao ZQ, Xiong W, Guo LB, Lu YF, Yi RX, Li JM, Yang XY, Zeng XY (2017) Fabrication of metal/semiconductor nanocomposites by selective laser nano-welding. Nanoscale 9(21):7012-7015

200. Yan XL, Wang F, Deng LM, Zhang CF, Lu YF, Nastasi M, Kirk MA, Li MM, Cui B (2018) Effect of laser shock peening on the microstructures and properties of oxide-dispersion-strengthened austenitic steels. Adv Eng Mater 20(3):1700641

201. Jiang YF, Ji B, Gan XD, Hua C, Li X, Zhu H (2018) Study on the effect of laser peening with different power densities on fatigue life of fastener hole. Opt Laser Technol 106:311-320

202. Shukla P, Crookes R, Wu H (2019) Shock-wave induced compressive stress on alumina ceramics by laser peening. Mater Des 167:107626

203. Luft A, Franz U, Emsermann L, Kaspar J (1996) A study of thermal and mechanical effects on materials induced by pulsed laser drilling. Appl Phys A 63(2):93-101

204. Kaspar J, Luft A (2001) Microstructure formed in body centred cubic metals by laser shock processing. Surf Eng 17(5):379-383

205. Hoppius JS, Kukreja LM, Knyazeva M, Pöhl F, Walther F, Ostendorf A, Gurevich EL (2018) On femtosecond laser shock peening of stainless steel AISI 316. Appl Surf Sci 435:1120-1124

206. Nakano H, Miyauti S, Butani N, Shibayanagi T, Tsukamoto M, Abe N (2009) Femtosecond laser peening of stainless steel. Laser Micro/Nanoeng 4(1):35-38

207. Park J, Han HS, Park J, Seo H, Edwards J, Kim YC, Ok MR, Seok HK, Jeon H (2018) Corrosion behavior of biodegradable Mg-based alloys via femtosecond laser surface melting. Appl Surf Sci 448:424-434

208. Kawashima T, Sano T, Hirose A, Tsutsumi S, Masaki K, Arakawa K, Hori H (2018) Femtosecond laser peening of friction stir welded 7075-T73 aluminum alloys. J Mater Process Technol 262:111-122

209. Fabbro R, Fournier J, Ballard P, Devaux D, Virmont J (1990) Physical study of laser-produced plasma in confined geometry. J Appl Phys 68(2):775-784

210. Sano T, Eimura T, Kashiwabara R, Matsuda T, Isshiki Y, Hirose A, Sano Y (2017) Femtosecond laser peening of 2024 aluminum alloy without a sacrificial overlay under atmospheric conditions. J Laser Appl 29(1):012005

211. Majumdar JD, Gurevich EL, Kumari R, Ostendorf A (2016) Investigation on femto-second laser irradiation assisted shock peening of medium carbon $(0.4 \%$ C) steel. Appl Surf Sci 364:133-140 